\title{
WestVirginiaUniversity
}

THE RESEARCH REPOSITORY @ WVU

Graduate Theses, Dissertations, and Problem Reports

2009

\section{Effects of response effort, delay, and stimulus changes on the sunk -cost effect}

James W. Diller

West Virginia University

Follow this and additional works at: https://researchrepository.wvu.edu/etd

\section{Recommended Citation}

Diller, James W., "Effects of response effort, delay, and stimulus changes on the sunk -cost effect" (2009). Graduate Theses, Dissertations, and Problem Reports. 2834.

https://researchrepository.wvu.edu/etd/2834

This Dissertation is protected by copyright and/or related rights. It has been brought to you by the The Research Repository @ WVU with permission from the rights-holder(s). You are free to use this Dissertation in any way that is permitted by the copyright and related rights legislation that applies to your use. For other uses you must obtain permission from the rights-holder(s) directly, unless additional rights are indicated by a Creative Commons license in the record and/ or on the work itself. This Dissertation has been accepted for inclusion in WVU Graduate Theses, Dissertations, and Problem Reports collection by an authorized administrator of The Research Repository @ WVU.

For more information, please contact researchrepository@mail.wvu.edu. 
Effects of response effort, delay, and stimulus changes on the sunk-cost effect

James W. Diller

Dissertation Submitted to the

Eberly College of Arts and Sciences

at West Virginia University

in partial fulfillment of requirements

for the degree of

Doctor of Philosophy

in

Psychology

Department of Psychology

Morgantown, West Virginia

2009

Karen G. Anderson, Ph.D., Chair

Kennon A. Lattal, Ph.D.

Michael Perone, Ph.D.

JoNell Strough, Ph.D.

William Trumbull, Ph.D.

Key words: sunk cost, escalation, choice, discriminative stimuli, delay, effort, pigeons. 


\begin{abstract}
Effects of response effort, delay, and stimulus changes on the sunk-cost effect James W. Diller
\end{abstract}

The sunk-cost effect is a decision-making fallacy that has its origins in the discipline in economics. In general, sunk-cost situations are typified by the presence of an initial investment that is followed by behavioral persistence, especially in the face of progressively worsening outcomes. This fallacy occurs when individuals use past expenditures (i.e., sunk costs) rather than future costs to guide decisions. Although there is a growing body of literature involving human participants, relatively little work has been done examining the variables that govern the sunk-cost effect in nonhuman subjects. The present experiments examined effects of response effort, delay, and stimulus changes on the sunk-cost effect in an animal model. In this procedure, pigeons responded on increasing ratio schedules of food reinforcement. In some conditions, the center keylight changed as the ratio increased (i.e., the increase was signaled). Responses on another alternative reset the ratio requirement to the lowest value, serving as an escape from the increasing ratios. In general, persistence was more likely to occur in the absence of the signals and when the delay to the onset of the next trial was relatively long or when the response requirement to escape was relatively high. 


\section{Acknowledgements}

I would like to thank Karen for her support throughout these past four-and-a-half years. It has been an invaluable learning experience to work with her, and I am grateful for the opportunities and guidance she provided for me. I would like to thank Andy and Mike for their voice of reason and scientific rigor; I am a better researcher and writer because of my experiences with each you. I would like to thank JoNell and Bill for adding unique perspectives to this project, and the insight in how to build connections between the basic lab and the broader world.

I am grateful for my friends from WVU who supported me throughout the process, especially Jon Slezak and Natalie Bruner, who were the best lab mates I could have asked for. I am thankful, too, for the undergraduate assistants (Ben Saunders, Tyson Sears, Hilary DeShong) that helped with the data collection, even after I moved on from Morgantown.

I thank my family and Laura for helping me and supporting me throughout this entire process, from West Virginia to Connecticut. 
Table of Contents

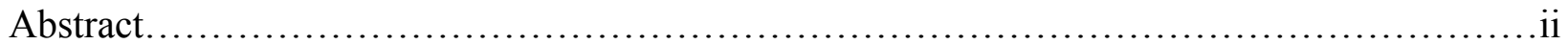

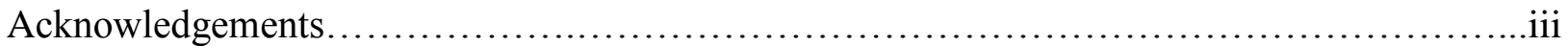

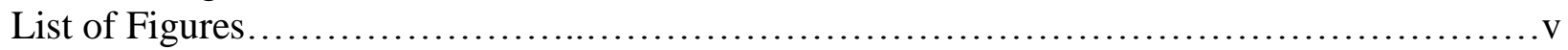

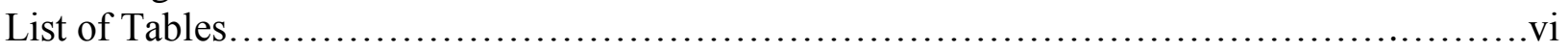

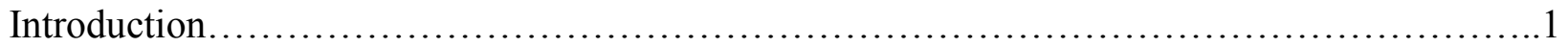

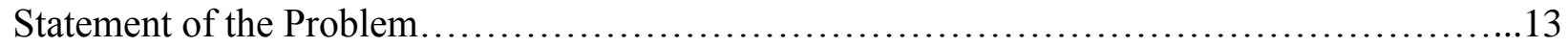

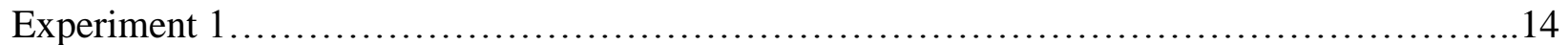

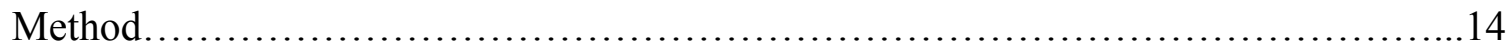

Subjects......................................................... 14

Apparatus..............................................................

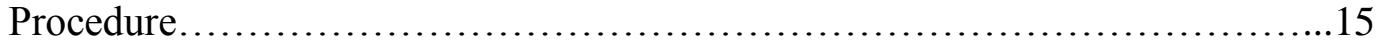

Initial Training....................................................

Subsequent Conditions............................................. 19

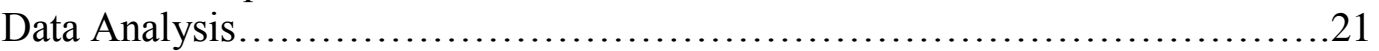

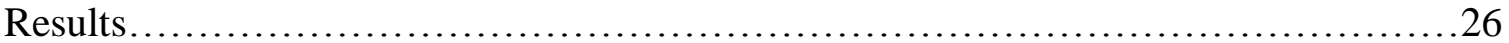

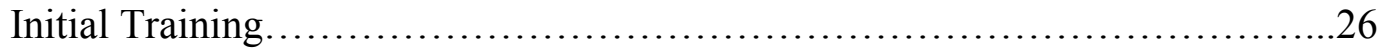

Experimental Manipulations ........................................... 30

Persistence-Optimal Conditions......................................... 31

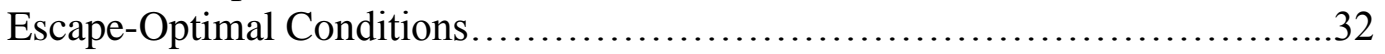

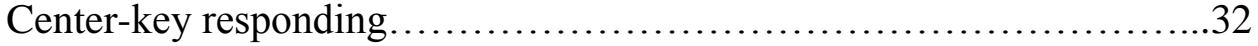

Center-key reinforcement......................................... 35

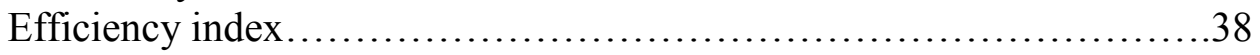

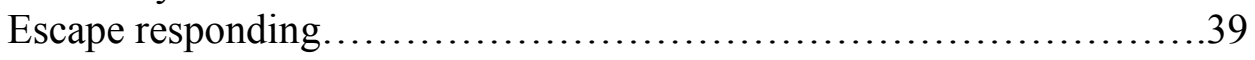

Condition reversal...............................................43

History comparison.............................................44

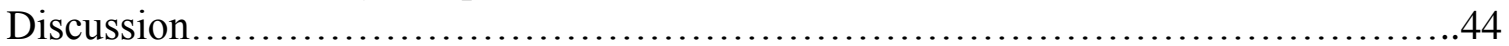

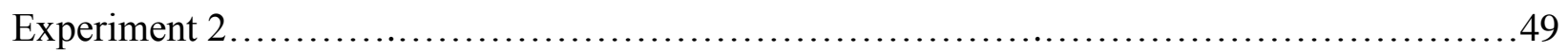

Method..................................................................50

Subjects \& Apparatus................................................ 50

Procedure.............................................................. 51

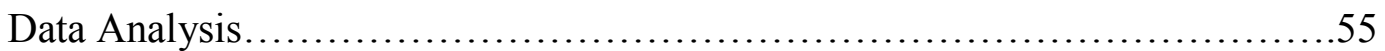

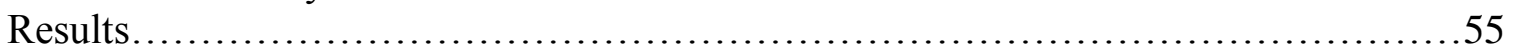

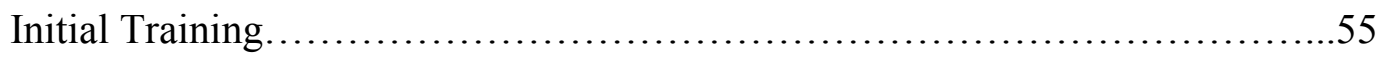

Experimental Manipulations..........................................57

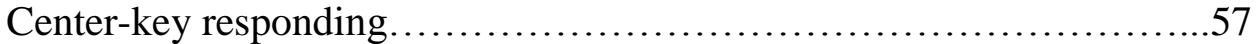

Center-key reinforcement....................................6 60

Efficiency index...........................................64

Escape responding............................................65

Condition reversal................................................69

Comparisons between experiments...................................... 70

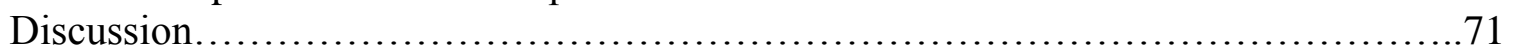

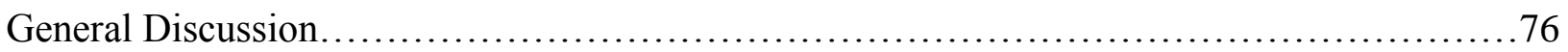

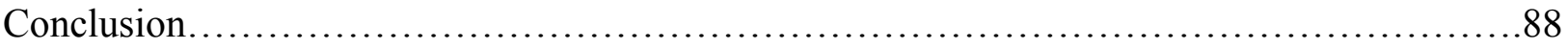

References........................................................................ 90 


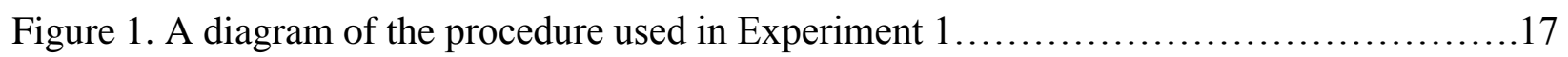

Figure 2. Center-key response rate (responses per minute), across conditions..................28

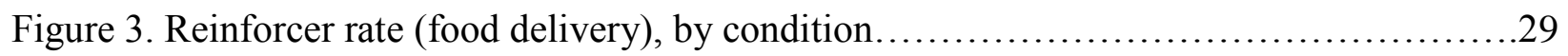

Figure 4. Efficiency index values, by subject and condition............................. 30

Figure 5. Mean number of completed escape responses, by condition........................33

Figure 6. Proportion of escape responses, across ratio values and conditions..................37

Figure 7. Proportion of food reinforcers delivered after the completion of each ratio...........42

Figure 8. A diagram of the procedure used in Experiment 2............................52

Figure 9. The center-key response rates (responses per minute) in each experimental condition,

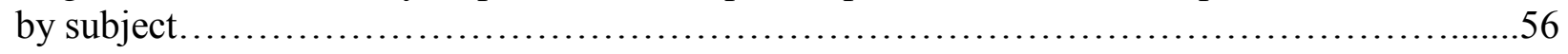

Figure 10. Efficiency index values, by subject and condition..............................58

Figure 11. Reinforcer rate (food delivery), by condition and subject. .....................59

Figure 12. Proportion of reinforcers delivered after the completion of each ratio..............63

Figure 13. Mean number of completed escape responses per session, by condition..............66

Figure 14. Proportion of escape responses, across ratio values and conditions................68 


\section{List of Tables}

Table 1. Variable-ratio values and probability statements...............................18

Table 2. Experimental conditions for two subjects in Experiment 1 that did experience persistence-optimal conditions, by subject number. The Optimal column indicates whether escaping or persisting resulted in the lowest mean response requirement. The Signals column indicates whether or not stimulus changes accompanied ratio changes. The escape requirement (an FR value) is indicated in the Escape column. Response rate on the center key (key pecks per minute), the number of escape responses per session, food reinforcement rate (reinforcers per minute), and the efficiency index are also presented. When signals were absent, the center key remained white. Note that the escape key was not available in the first condition (i.e., initial training) and the second condition for Subject A3. The symbol ${ }^{\circ}$ denotes that sessions ended with 40 grain deliveries rather than 80 . The symbol ${ }^{\ddagger}$ indicates that the condition was not run to stability because of concerns that extinction of the escape-key response may have occurred. To foster the recovery of this response, this condition was conducted briefly. This symbol * indicates that modified stability criteria (e.g., 20\% variance from the grand mean, or fewer than 10 escape

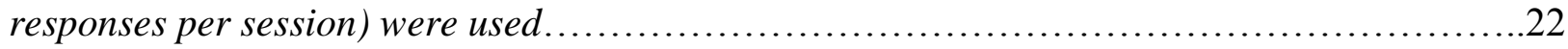

Table 3. Experimental conditions for the two subjects that did not experience persistence-optimal conditions in Experiment 1, by subject. The Signals column indicates whether or not stimulus changes accompanied ratio changes. When signals were absent, the center key remained white. The escape requirement (an FR) is indicated in the Escape column. Response rate on the food key (key pecks per minute), the number of escape responses per session, food reinforcer rate (reinforcers per minute), and the efficiency index are also presented. Note that the escape key was not available in the first condition (i.e., initial training). This symbol * indicates that modified stability criteria (e.g., 20\% variance from the grand mean, or fewer than 10 escape responses per session) were used .................................................24

Table 4. Conditions in Experiment 2, by subject. The Signals column indicates whether or not stimulus changes accompanied ratio changes. When signals were absent, the center key remained white. The Delay column indicates the delay between the escape response and the onset of the next trial. Note that the escape key was not available in the first condition (i.e., initial training). The Response Rate column contains the mean number of center-key pecks per minute. The rate of food reinforcement is presented in reinforcers per minute. Obtained efficiency index values are presented in the final column. The symbol ${ }^{\ddagger}$ indicates that the condition was not run to stability because the subject (due to the amount of weight gained in each experimental session) was unable to run daily. Thus, to increase the likelihood that this subject could run daily, the number of reinforcer deliveries was decreased from 80 to 40. The symbol ${ }^{\circ}$ denotes that sessions ended with 40 grain presentations. This symbol indicates that modified stability criteria (e.g., 20\% variance from the grand mean, or fewer than 10 escape

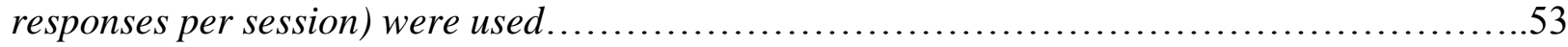


Navarro and Fantino (2005) defined the sunk-cost effect as "the tendency to persist in an endeavor once an investment of effort, time, or money has been made" (p. 1). Sunk cost is an economic concept that suggests that investments are likely to continue based on past expenditures. Navarro and Fantino suggested that this type of behavioral persistence may be suboptimal if the situation becomes worse, as when additional resources are required to achieve the goal at hand. When the sunk-cost effect is exhibited, individuals persist in a worsening situation rather than escaping and pursuing a different course of action. Sunk-cost behavior may be maladaptive in that it is a failure to optimize reinforcement, wasting resources while other (perhaps more profitable) courses of action are not pursued.

In an ideal decision-making situation, past investments should not be considered—only future costs should enter into choices (Arkes \& Ayton, 1999). Humans and other animals do not always engage in this optimal behavior, however, often evaluating choice outcomes in terms of past expenditures. Failure to consider future costs could result in clinically significant problems, as when an individual stays in an abusive relationship based on prior time investment (Leahy, 2000). The sunk-cost effect is typified in situations in which behavior persists once some resource (e.g., money, time, effort) has been expended to achieve a particular goal. This effect has been studied extensively in humans (cf. Arkes \& Ayton, 1999; Arkes \& Hutzel, 2000; Bornstein \& Chapman, 1995; O’Flaherty \& Komaki, 1992; Strough, Mehta, McFall \& Schuller, 2008) but, until relatively recently, had not been examined in nonhuman animals (Navarro \& Fantino, 2005).

Variables that have been implicated in the sunk-cost effect include the nature of the initial investment (e.g., the specific commodity and/or the amount) and situational variables such as the salience of the choice situation, the degree of personal responsibility of the decision-maker, and 
the degree of uncertainty in the investment situation (Bornstein \& Chapman, 1995; McCain, 1986). These two classes of variables have been shown to affect choice and the likelihood that the sunk-cost effect will occur. Successful experimental analysis of the sunk-cost effect requires manipulation of both types of variables. Fantino (2004) suggested that decision making is governed by "historical and contemporary contingencies of reinforcement" (p. 287). The two experiments of this dissertation provide analyses of the historical and contemporary contingencies that govern the sunk-cost behavior described by Navarro and Fantino (2005).

The belief that a particular endeavor will be successful is one variable implicated in the sunk-cost effect (Arkes \& Hutzel, 2000). Arkes and Hutzel (2000) suggested that the presence of a sunk cost (i.e., previous expenditure) results in an increased likelihood that behavior will persist when the sunk cost is considered in conjunction with the estimated probability of successful task completion. In this study, participants were presented with written scenarios and the option to invest funds into a particular project with varying sunk costs (i.e., levels of previous expenditure) with either the presence or absence of a prescribed probability of success. Participants were more likely to continue investing when the sunk cost was high. Arkes and Hutzel also suggested that the perceived probability of success may be inflated as behavior continues, perhaps increasing the likelihood that the behavior will recur. The probability of success may be related to the degree of certainty associated with the desired outcome.

Uncertainty was implicated by Navarro and Fantino (2005) as a factor that influences sunk-cost behavior, drawing on findings from McCain (1986). In his study, McCain presented undergraduates with an investment protocol in which they could increase or decrease investment in an unprofitable research-and-development project. McCain found that the participants were likely to increase investment (i.e., persist) initially, despite losses, but, as losses increased, they 
would decrease their investment. McCain suggested that the initial escalation could be due to the unclear nature of the situation; when the costs became more salient, de-escalation did occur, and optimal behavior (i.e., less waste of resources) was observed. In the present experiments, both the probability of success (i.e., ratio completion) and the degree of certainty were manipulated.

As the number of behavioral repetitions increases, it has been hypothesized that the net impact of each repetition on future behavior is lessened. That is, each behavior accounts for a smaller proportion of the whole set of responses, and, therefore, has a proportionately diminished effect. One model of this phenomenon is known as Bayesian updating. O'Flaherty and Komaki (1992) evaluated the role of Bayesian updating in decision-making processes. Bayesian updating is defined as evaluating the likelihood of future behavior based on the occurrence of past behavior on a trial-by-trial basis (e.g., using the outcome of trial 8 to predict the outcome of trial 9). This type of analysis may be applied to decision-making models and suggests that new, incoming information can influence consecutive decision-point actions. As new information comes in, the decision-making process is updated, and the topography of the choice situation is altered. For example, if an investment has been made in a particular fund and that fund fails to yield a profit, individuals may incorporate this information when presented with the choice to continue investing at the current level or to change investment strategies. This model can also be used to explain persistence of a behavior that continues in the absence of its maintaining consequences (i.e., extinction). When extinction is in effect, past outcomes influence future behavior in a probabilistic fashion, and Bayesian modeling can be used to predict peaks of responding in the extinction curve based on the organism's reinforcement history.

Another major facet of Bayesian updating is that as time passes, each instance of feedback has less impact on the behavioral outcome. When there are relatively few instances of 
feedback, each instance constitutes a large proportion of the net feedback. As the number of trials increases, the proportion of the total effect accounted for by each trial necessarily decreases. Thus, with increasing numbers of observations, each individual observation has a lessened impact on the decision-making process in general and becomes less of a determinant of choice (O’Flaherty \& Komaki, 1992). Bayesian modeling suggests that situations in which sunk costs have been expended may have been profitable at one time, but the influence of these historical occurrences diminishes as time passes. Behavior may continue as it had before, even when the prevailing contingencies have changed. It logically follows that increased variability in outcomes may lead to an increased likelihood of sunk-cost behavior. This assumption was examined in the present experiments.

The notion that prior investment impacts behavioral persistence was evaluated by Goltz (1999). This topic was explored using a model of investment in which participants received good or bad feedback related to investment decisions (i.e., gains or losses). After receiving feedback, participants could adjust the amount of money invested. In this study, the degree of persistence (continuing to invest) or escalation (investing more) was directly related to outcomes in the initial training component. Specifically, initial positive outcomes (e.g., gains) led to a longer period of investment and more escalation than did mixed or negative outcomes (e.g., losses). Here again, past investment influenced the likelihood of behavioral persistence in the face of worsening situations.

Moon (2001) suggested that persistence and escalation (i.e., increasing commitment to a particular course of action) can be explained based on both sunk cost and the degree of current project completion. Using a survey, Moon found that individuals considered both the past outcomes and the hypothetical future before committing to a particular decision or course of 
action. In this study, participants did consider future costs before making their decisions, indicating that they did not exclusively demonstrate the sunk-cost effect. Because the past investment influenced future investment decisions, however, behavior was not fully rational (as defined by Arkes \& Ayton, 1999) and the sunk-cost effect was exhibited. In the present experiments, the salience of the degree of completion was manipulated.

Rachlin (2004) discussed the notion of "soft commitment," which he defined as the increased likelihood of persistence after an initial response has been made. Although it has not been previously described as such, soft commitment bears similarities to a sunk-cost situation. Soft commitment is distinct from a strict-commitment response in that making an initial response on one alternative does not preclude making a response on the other. Rather, the behavior can change or be altered, but Rachlin suggested that changing an established pattern of behavior is associated with a cost, so behavior is likely to persist uninterrupted. In an empirical study, Siegel and Rachlin (1995) evaluated effects of soft commitment within a self-control paradigm: the choice between a smaller, more immediate reinforcer and a larger, delayed reinforcer (cf. Ainslie, 1974; Mazur, 1987). Pigeons served as subjects in this experiment, and a fixed-ratio (FR) 31 schedule of food reinforcement was arranged across two keys. In this procedure, the first 30 responses could be delivered on either alternative (i.e., the side key associated with the smaller, sooner reinforcer or the side key associated with the larger, later reinforcer) without determining the outcome. It was the thirty-first response that determined which reinforcer (large or small) was delivered. When the FR 31 schedule was in place, the larger reinforcer was chosen more often than when an FR 1 schedule was in effect for either alternative (i.e., one response resulted in delivery of either the small or large reinforcer) or when a 30-s fixed-interval (FI) schedule was in effect for both outcomes. The effect of the FR 31 schedule (i.e., increasing large- 
reinforcer choice) was augmented when a signal (a brief change in key color) was presented immediately before the thirty-first response. The presentation of this signal resulted in an increased likelihood that the self-controlled response (i.e., selecting the larger, delayed reinforcer) would be made, indicating that signals may enhance the efficiency of behavior.

Overall, the results published by Siegel and Rachlin (1995) suggest that a softcommitment response increased the likelihood that subjects would receive the larger, delayed reinforcer, relative to phases in which the commitment response was not present. Additionally, Siegel and Rachlin suggested that once behavioral patterns are established, interruption (i.e., non-persistence) of these patterns may be associated with a cost. Coupled with the influence of the stimulus change (signal), the data presented by Siegel and Rachlin support the findings of Navarro and Fantino (2005). Specifically, responding may persist once it has begun because there are costs associated with changing courses of action.

Navarro and Fantino (2005) suggested that their study was the first to establish a behavior-analytic model of the sunk-cost effect. In this model, FR schedule requirements increased within each trial based on a series of probability statements. In a series of experiments, subjects (pigeons and humans, working for grain and money, respectively) had the opportunity to continue responding on one ratio or to escape from the current schedule with the possibility that a lower programmed ratio may result (i.e., resetting the ratio requirement). The probability that a given ratio would result in food (or money) delivery was determined independently for each trial, and satisfying the lowest ratio resulted in food delivery for half of the trials. Navarro and Fantino systematically examined effects of different probability arrangements and the presence or absence of correlated stimulus changes (signals) on patterns of persistence and resetting. When higher response requirements were in effect and these requirements were signaled, subjects were 
more likely to reset (i.e., escape from the current ratio) when doing so resulted in a lower mean response requirement (i.e., was optimal). When stimulus changes were absent, subjects were more likely to behave suboptimally, persisting and subsequently emitting more responses than were necessary to obtain the food or money. This was described as engaging in sunk-cost behavior. Navarro and Fantino's description of the sunk-cost effect is directly relevant to the notion of soft commitment advanced by Rachlin in that established behavioral patterns (without interruption or stimulus changes) were likely to continue. When stimulus changes were present, however, subjects were likely to reset the ratio requirement.

As a follow-up to their 2005 study, Navarro and Fantino (2007) further explored the importance of the discriminability of worsening situations in the sunk-cost effect. In this study, human participants engaged in a computer task in which they were responding for hypothetical monetary rewards. Trials began with a relatively short ratio requirement, which increased throughout each trial. The ratio requirements used in this study were the same as in the Navarro and Fantino (2005) experiments and in the present procedure (10, 40, 80 and 160 responses, in effect for $50 \%, 25 \%, 12.5 \%$, and $12.5 \%$ of trials, respectively). Participants had the option to continue to respond on a given trial, or could reset the ratio requirement. To assess effects of discriminative stimuli on persistence in this paradigm, a between-groups design was employed. For one group, as lower ratios were completed (but did not result in money delivery), the computer screen changed colors. For the second group, the screen remained white throughout each trial. Participants presented with stimulus changes were more likely to escape (i.e., less likely to persist) than the participants in the group without stimulus changes. The results from this study underscored the importance of discriminative stimuli in sunk-cost behavior.

Since the publication of Navarro and Fantino's (2005) initial study, several other 
researchers have examined effects of past investment (e.g., previous responses to a given alternative) on choice in nonhuman species. For example, Pompilio and Kacelnik (2005) presented a study examining the relation between state-dependent learning and suboptimal choice. In this study, food was initially delivered to starlings when they were prefed or food deprived, and each state of deprivation was correlated with a different colored light. In choice conditions, regardless of their present state (deprived or prefed), the starlings preferred (as indexed by key pecks allocated to each alternative) the alternative that was initially associated with their state of greater food deprivation. In this study, the initial experience with the alternatives (when subjects were food deprived or prefed) may be described as the initial investment, or sunk cost.

After Pompilio and Kacelnik observed the preference for the alternative that was initially presented in a state of deprivation, they increased the delay to food presentation for both sources to $10 \mathrm{~s}$. At a 10-s delay, preference continued for the alternative correlated with initial deprivation, and the delay to the presentation of the food from the source was increased further across experimental sessions. When the delay to the food source that was first contacted while the subjects were deprived reached $17.5 \mathrm{~s}$, subjects responded equally for each alternative; prior to that delay value, however, preference for the deprivation-associated alternative was maintained. In this study, the authors associated a longer delay with an increasing cost, and as the delay increased, choice became less optimal (e.g., the reinforcement rate was not maximized because the further-delayed alternative was not selected). Pompilio and Kacelnik suggested that the subjects were basing their choice on their history of responding past responses rather than the future cost (i.e., delay) associated with each alternative. By failing to consider future costs, subjects were engaging in suboptimal patterns of behavior, in that the rate of reinforcement was 
not maximized.

In another study, Pompilio, Kacelnik, and Behmer (2006) implicated prior investment as a determinant of choice using desert locusts (grasshoppers) as subjects. In this study, the deprivation state of the desert locusts was manipulated as they chose between two odors (peppermint and lemon grass). In training, one odor was presented in a high state of food deprivation, and the other odor was presented in a low state of food deprivation, and both odors were paired with the delivery of a piece of wheat. In choice tests, the high-deprivation odor was selected more frequently than the low-deprivation odor, regardless of the current state of deprivation during testing. As in the study with the starlings, the initial experience with the alternatives controlled subsequent response allocation, even though the outcome of selecting one alternative or the other was the same (e.g., the delivery of a piece of wheat). Unlike the study with the starlings, delays were not introduced in the choice situation. Even though delay was not manipulated, the preference for the high-deprivation alternative by grasshoppers underscores the role of the initial context in subsequent choices.

Although the current discussion has focused on the analysis of behavioral persistence in terms of the sunk-cost effect, it is also possible to conceptualize these findings in other domains. For example, the scheduling of escalating response requirements and the option to escape to a lower requirement relates to work examining diminishing returns (cf. Wanchisen, Tatham, \& Hineline, 1988; Hackenberg \& Hineline, 1992; Hackenberg \& Axtell, 1993; Jacobs \& Hackenberg, 1996) and choice between ratio schedules (cf. de la Piedad et al., 2006).

Generally, in diminishing-returns procedures, subjects are presented with a choice between concurrently available fixed and progressive schedules of reinforcement. The selection of one of the schedules prevents responding on the other. Each time the progressive schedule is 
chosen, the associated schedule requirement increases. The schedule requirement on the fixed option remains the same throughout individual sessions, but may vary between experimental conditions. In some conditions, the selection of the fixed schedule results in the resetting of the progressive value; in others, selection of the fixed schedule has no consequence for the arrangement of the progressive schedule. Within these studies, the point at which responding switches from the progressive to fixed schedule is a primary dependent variable of interest. This may be similar to Navarro and Fantino's sunk-cost procedure, in which the point at which the subject resets to the lowest ratio value is of interest.

In a study described by Hackenberg and Hineline (1992), pigeons chose between an FI schedule of reinforcement and a progressive-interval (PI) schedule of reinforcement. This was in contrast to previous diminishing-returns research in which ratio schedules of reinforcement were used (cf. Wanchisen, Tatham \& Hineline, 1988). In the procedure used by Hackenberg and Hineline, when subjects began to respond on one of the two schedules, the other operandum was disabled, and when food was delivered according to the PI schedule, the PI value increased by 20 s on the next trial. In some conditions, the completion of the FI schedule resulted in the resetting of the PI schedule to its initial value. When FI completion resulted in resetting the PI schedule, the switching values (from the PI schedule to the FI schedule) were lower than when the resetting contingency was not in place. Additionally, switches occurred at lower PI values when the FI values were relatively short. The results of this study demonstrated control of behavior by temporally distal consequences (e.g., increasing PI requirements), and also demonstrated similarities in the patterns of switching between progressive and fixed ratio and interval schedules.

When conceptualizing the diminishing-returns literature, Hineline and Sodetz (1987) 
described the diminishing-returns procedures as an analogue to a foraging task. In foraging studies, choice is between a diminishing patch (the progressive schedule) and a new patch (the fixed schedule). Because of the conceptual ties to the foraging literature, it is possible to make predictions with respect to the diminishing-returns literature based on maximization. As Hackenberg and Hineline (1992) discussed, two assumptions should hold true: (1) persistence on the progressive schedule should vary as a function of the value of the fixed schedule, and (2) the points of transition should maximize overall reinforcer rates. Because the sunk-cost procedure also involves choice to continue working under diminishing returns (e.g., a higher ratio requirement) or to start over with a lower work requirement, it may be possible to extend these predictions into the sunk-cost literature. Specific translation of these assumptions state that: (1) behavioral persistence should vary as a function of the escape requirements, with increasing escape requirements resulting in increased behavioral persistence and (2) the point of escaping should maximize overall reinforcement.

Two factors that influence foraging behavior that may be relevant in an examination of sunk-cost behavior are travel time and handling time (cf. Fantino \& Abarca, 1985). Travel time is conceptualized the delay associated with changing between patches. In the sunk-cost procedure developed by Navarro and Fantino, the travel time may be conceptualized as the intertrial interval (ITI) after an escape response or food delivery. When travel time has been increased in experimental studies (cf. Dunn, 1982), switching between patches decreased (i.e., persistence on a given patch increased). Relating this to the sunk-cost procedure, as the ITI increases, it is expected that persistence should increase proportionally.

Handling time may be defined as the time required to obtain food. In the sunk-cost procedure, this may be conceptualized as the response requirement. As the subject progresses 
through each trial and is faced with an increasing response requirement, the handling time increases. Research in the area of foraging has demonstrated that increasing handling time results in decreased selectivity, i.e., increased choice for the more easily available reinforcer (Ito \& Fantino, 1986). In the sunk-cost procedure, as described by Navarro and Fantino, decreased selectivity may be demonstrated by decreased rates of escape responding. That is, if a response requirement or a delay is increased in the sunk-cost procedure, a decrease in escape responses would be consistent with the decrease observed in the foraging paradigm.

Besides the diminishing-returns and foraging literatures, other types of studies have examined effects of schedule parameters on choice between schedules. For example, de la Piedad et al. (2006) assessed effects of past choices on future behavior. In the first experiment of this study, pigeons were presented with a choice between a random-interval (RI) 60-s schedule and a tandem continuous-reinforcement, FI 14-s schedule (CRF-FI). In a choice situation in which responding on the CRF-FI schedule prevented future responses on the RI schedule, subjects were likely to continue responding on the RI schedule. When responding on the RI schedule continued, the overall obtained reinforcement rate was not maximized. The authors found that future responses on the RI schedule depended on the pigeons' past choices to respond on this schedule. The results from the study by de la Piedad et al. (2006) exemplify the impact of past choices on future behavior.

As described above, there are a variety of topics that can be related to the sunk-cost effect. The present experiments were designed to contribute to the analysis of this effect by systematic manipulation of parameters to identify conditions under which sunk-cost behavior is observed. The exploration of the impact of variables within the controlled setting of the animal lab may allow for the identification of the factors contributing to human sunk-cost behavior. This 
work may ultimately lead to the development of interventions to decrease this type of maladaptive persistence.

\section{Statement of the Problem}

Navarro and Fantino (2005) provided the first behavior-analytic analysis of the sunk-cost effect in nonhuman animals. These authors found that behavior was more likely to persist in worsening situations when the worsening conditions were less discriminable. This notion is congruent with Bornstein and Chapman's (1995) assertion that the salience of the choice situation is an important variable in the sunk-cost effect. One method of disrupting the discrimination between prevailing contingencies is to use variable-ratio (VR) schedules of reinforcement instead of FR schedules of reinforcement. The use of VR schedules also affects the probability that each response will result in the delivery of grain, tapping into the probability of success variable implicated by Arkes and Hutzel (2000). A second means of manipulating the discriminability of the prevailing contingencies is to include or remove stimulus changes correlated with the increasing ratio requirements. Effects of the presence and absence of correlated stimulus changes and fixed- and variable-ratio schedules were assessed in the present experiments.

The response requirement to reset the ratio in effect (i.e., escape) was also manipulated in the present study. This variable is implicated in the literature that demonstrates that perceived cost (as expressed by the probability of success, or proximity to completion) is an important factor contributing to behavioral persistence in human subjects (cf. Arkes \& Hutzel, 2000; Bornstein \& Chapman, 1995). Because increasing the response requirement necessarily increases the delay to the onset of the next trial, effects of increasing the delay between the escape response and the onset of the next trial were also examined. This manipulation allowed 
evaluation of the extent to which the sunk-cost effect increases as a function of an increased delay to the onset of the next trial as opposed to an increased escape-response requirement.

The present experiments were designed to replicate and extend the work of Navarro and Fantino (2005). Four variables were manipulated in two experiments: (1) the type of ratio schedules (fixed or variable); (2) the presence and absence of signals correlated with the increasing response requirement; (3) the number of responses required on the escape key; (4) the delay between the escape response and the onset of the next trial. The systematic manipulation of these four variables may facilitate identification of conditions under which sunk-cost behavior, as described by Navarro and Fantino, may be observed.

\section{Experiment 1}

Method

\section{Subjects}

Four experimentally experienced male White Carneau pigeons with largely unknown histories served as subjects in this experiment. They had continuous access to water and grit in their home cages and were maintained at approximately $80 \%$ of their ad-libitum weight. Due to sufficient quantities of food earned in each experimental session, little or no post-session feeding was necessary. Thus, subjects were typically maintained on a closed economy.

\section{Apparatus}

Four operant-conditioning chambers located in sound-attenuating enclosures were used in this experiment. Each chamber measured $305 \mathrm{~mm}$ wide by $335 \mathrm{~mm}$ deep, and was $375 \mathrm{~mm}$ tall. An aluminum panel comprised the front wall of the chambers. Three translucent plastic keys, 25 $\mathrm{mm}$ in diameter, were located on the aluminum panel, $265 \mathrm{~mm}$ from the chamber floor. The centers of the keys were $90 \mathrm{~mm}$ apart, and the leftmost key was $70 \mathrm{~mm}$ from the left edge of the 
chamber. Each key could be transluminated by four or five $28-\mathrm{V}$ miniature lamps that were covered by colored lens caps. Mixed grain was provided by a food hopper that was raised into a 65- by 55-mm opening in the front panel of the chamber. The bottom edge of the opening was located $180 \mathrm{~mm}$ below the center of the center key. A white lamp above the food hopper was illuminated when grain was presented. A ventilation fan circulated air and masked extraneous noise throughout the experimental session. All experimental events were controlled by a computer running MedAssociates ${ }^{\circledR}$ software, and programs were written by the author using MedState ${ }^{\circledR}$ notation.

\section{Procedure}

Initial training. Experimental sessions were conducted five to seven days per week at approximately the same time each day. All sessions began with a 10-min blackout period before the onset of stimuli. Most sessions ended with 80 reinforcer deliveries, but, for some subjects, it became necessary to reduce the number of reinforcers to 40 , as noted below.

The first condition of this experiment involved increasing FR schedules of grain delivery with correlated stimulus changes in effect on the center key, which was the only active key during this condition. The ratio values that were used in the initial condition were the same as the mean ratio values used in subsequent conditions, when the schedule arrangement was changed from FR to VR. The ratio values were 10, 40, 80, and 160 (described as low, medium, high, or highest, respectively), and they were arranged according to a series of probability statements, as described below. A distinct key color was correlated with each ratio value (white for 10, yellow for 40 , green for 80 , and red for 160), and these colors were also used in subsequent signaled conditions. After the initial condition, two procedural changes occurred. First, VR schedules were used instead of FR schedules, with mean VR values that were the same as the initial FR 
values. And, second, the right (escape) key became active; see below.

At the start of each trial (in both initial and subsequent conditions), the ratio in effect (i.e., the number of pecks required before $3 \mathrm{~s}$ of grain access were provided) was selected based on a series of probability statements (described below). Each trial began with the onset of a white center keylight, which was correlated with the low ratio. When one of the higher ratios was selected by the computer program as the terminal ratio for a given trial, completion of the lower ratios resulted in a change of the center key color, signaling an increase in the ratio requirement. The changing keylight color (the signal) was present in the initial training component and some of the later conditions. There were also phases in which the center key remained white throughout the session (unsignalled phases).

A diagram of the procedure is presented in Figure 1. As in Navarro and Fantino's (2005) escape-optimal conditions, the terminal ratio for each trial was independently selected according to probability statements. Unlike the procedure used by Navarro and Fantino, however, VR schedules (rather than FR) were programmed; see Table 1 for VR values and probability statements. All trials began with a VR 10 in effect on the center key. On half of the trials $(\mathrm{p}=.5)$, $3 \mathrm{~s}$ of access to grain were provided following the completion of the VR 10 (Figure 1, dotted arrows). For the other half of trials, one of the larger ratio requirements $(40,80$, or 160) was in effect, and additional responses were required before grain was delivered. If, at any point in time, subjects responded on the escape key (Figure 1, dashed line), a 1-s delay (blackout) occurred, followed by the onset of the next trial. The number of pecks required to initiate an escape response was systematically varied across experimental conditions. The completion of the selected terminal ratio resulted in $3 \mathrm{~s}$ of access to grain, followed by a 1-s blackout (i.e., a 1-s ITI) and the illumination of the center and right keys. At the onset of the next trial, the lowest 
Figure 1.

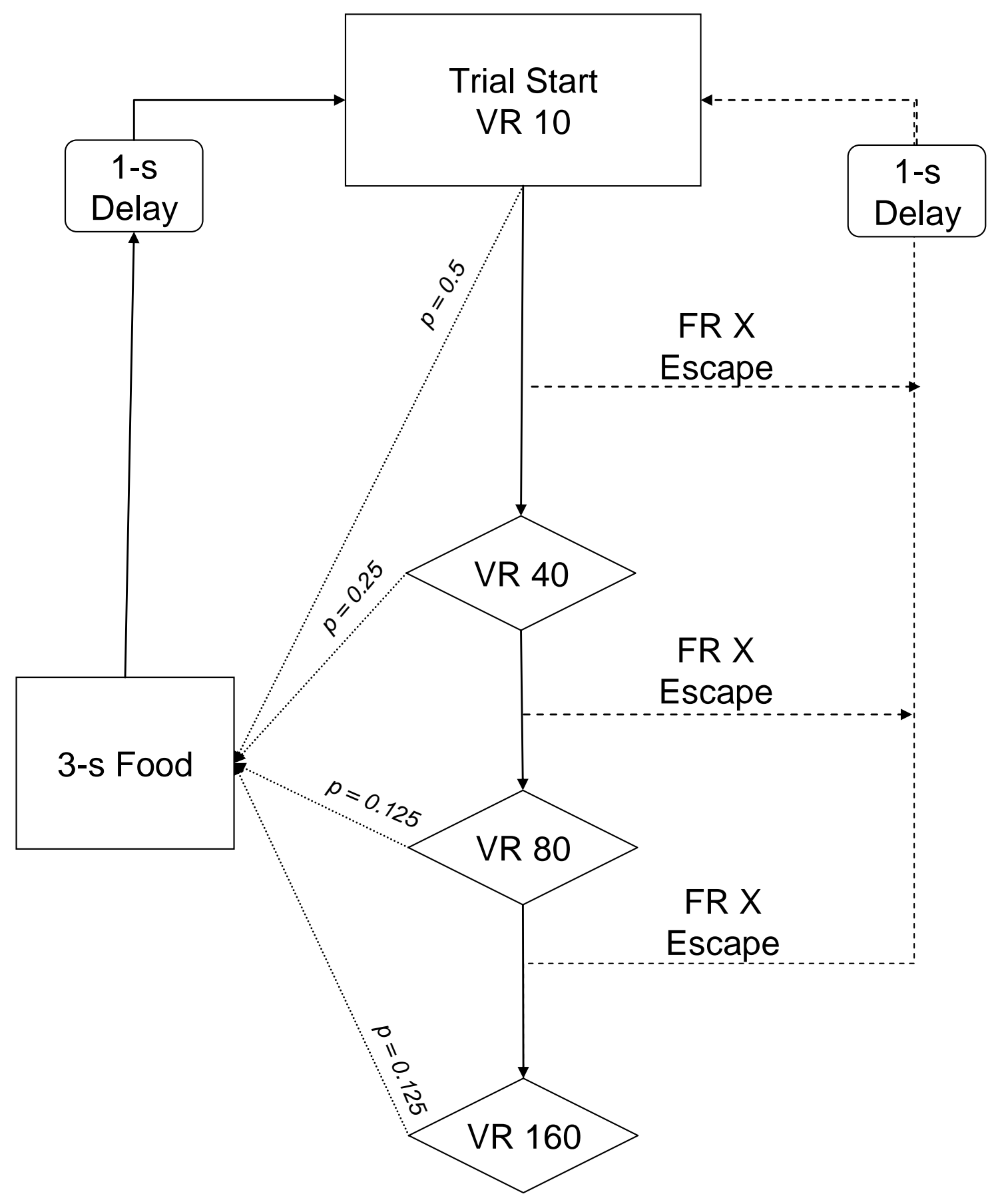


Table 1.

Variable-ratio values and probability statements.

\begin{tabular}{|c|c|c|c|c|}
\hline Condition & $\begin{array}{l}\text { Key } \\
\text { Color }\end{array}$ & $\begin{array}{l}\text { Mean } \\
\text { VR }\end{array}$ & Probability & Values \\
\hline $\begin{array}{l}\text { Escape } \\
\text { Optimal }\end{array}$ & White & 10 & $\mathrm{p}=.500$ & $\begin{array}{l}1,2,3,4,5,6,7,8,9,10,11,12,13,14 \\
15,16,17,18,19\end{array}$ \\
\hline $\begin{array}{l}\text { Escape } \\
\text { Optimal }\end{array}$ & Amber & 40 & $p=.250$ & $\begin{array}{l}20,21,23,24,31,32,33,39,40,42,44, \\
45,50,51,52,55,60\end{array}$ \\
\hline $\begin{array}{l}\text { Escape } \\
\text { Optimal }\end{array}$ & Green & 80 & $\mathrm{p}=.125$ & $61,62,64,65,69,76,80,91,93,139$ \\
\hline $\begin{array}{l}\text { Escape } \\
\text { Optimal }\end{array}$ & Red & 160 & $\mathrm{p}=.125$ & $\begin{array}{l}140,143,144,150,155,160,160,168 \\
180,200\end{array}$ \\
\hline $\begin{array}{c}\text { Persistence } \\
\text { Optimal }\end{array}$ & White & 10 & $\mathrm{p}=.250$ & $\begin{array}{l}1,2,3,4,5,6,7,8,9,10,11,12,13,14, \\
15,16,17,18,19\end{array}$ \\
\hline $\begin{array}{c}\text { Persistence } \\
\text { Optimal }\end{array}$ & Amber & 30 & $\mathrm{p}=.583$ & $\begin{array}{l}20,21,22,23,24,25,26,27,28,29,30, \\
31,32,33,34,35,36,37,38,39,40\end{array}$ \\
\hline $\begin{array}{l}\text { Persistence } \\
\text { Optimal }\end{array}$ & Green & 50 & $\mathrm{p}=.167$ & $41,43,45,47,48,49,55,58,58,60,62$ \\
\hline
\end{tabular}


ratio requirement was again in effect, and center key was transluminated with white light. Subjects could then respond on the lowest ratio schedule to begin working towards the completion of the selected terminal ratio for that trial, as determined by the probability statements.

Training with increasing ratio values without the escape key available continued until stable rates of pecking on the center key (i.e., the food key) were observed. Data were considered stable when, in the final nine sessions divided into three blocks of three sessions, there were no increasing or decreasing trends across the blocks and no more than $15 \%$ variability of each mean relative to the overall mean. For some conditions in which response rates were low, modified stability criteria of no more than $20 \%$ variability from the grand mean, or a total of 10 or fewer escape-key responses, were used. Each experimental condition continued until the stability criteria were satisfied or until 40 sessions occurred.

\section{Subsequent Conditions.}

After stability was attained in the initial training procedure, the right key (i.e., escape key) was activated, and the number of responses required on this key to reset the ratio in effect on the center key was systematically manipulated across conditions. In the first condition, a single key peck was required on the right key to reset the center-key ratio requirement to the lowest value. In subsequent conditions, this value was increased to five responses and then increased by five responses per condition.

Effects of the presence and absence of changing key colors (i.e., signals) correlated with increasing ratio requirements were assessed at each escape-requirement value. That is, the key color changes were in effect initially and were removed in a second condition with the same right-key ratio requirement. If response rates on the key correlated with food delivery or the 
overall number of escapes changed between the signaled and unsignalled conditions, the signals were reintroduced with the same escape requirement in a third condition. Whether behavior changed or remained stable across these conditions was determined by applying the stability criteria (above) to the final nine sessions. If no difference was observed between the final nine sessions of the two conditions (signaled, unsignalled) the reversal to the signaled condition was not conducted.

As in the experiments of Navarro and Fantino (2005), the scheduling of the food delivery was manipulated in the present study. This manipulation took the form of escape-optimal conditions or persistence-optimal conditions. Similar to the use by Navarro and Fantino, 2005, optimality, here, refers to the pattern of responding that would most efficiently result in grain delivery (i.e., with the fewest responses per reinforcer). Two subjects began this experiment in escape-optimal conditions and two began in persistence-optimal conditions. During escapeoptimal conditions, grain delivery was scheduled as described above. As in the procedure used by Navarro and Fantino (2005), the schedules of grain delivery in persistence-optimal conditions were arranged as follows: On three-twelfths of all trials $(\mathrm{p}=.25)$, a VR-10 schedule was in effect. For seven-twelfths of all trials $(\mathrm{p}=.583)$, a VR-30 schedule was in effect. For twotwelfths of all trials $(\mathrm{p}=.167)$, a VR-50 schedule was in effect. Variable ratio values are presented in Table 1 . The completion of the selected terminal ratio value resulted in $3 \mathrm{~s}$ of access to grain, followed by a 1-s ITI and the illumination of the center and right keys, indicating that the next trial had begun. At the onset of each trial, the lowest ratio requirement was again in effect. At this point, subjects could respond to complete the lowest ratio, working towards the completion of the selected terminal ratio for that trial (as determined by the probability statements). This procedure was in effect until the stability criteria (as described above) were 
satisfied for both escape responding and center-key responding.

After subjects completed three phases with persistence-optimal conditions (signaled, unsignalled, signaled), escape-optimal conditions were in effect for the remainder of the study. The order of conditions experienced by the subjects beginning with the persistence-optimal conditions are presented in Table 2 and the order of the conditions experienced by the other two subjects is presented in Table 3 .

\section{Data analysis.}

The primary dependent measures of this experiment were the response rate on the center key, the number of completed escape responses, and the reinforcement rate. The efficiency index was used to assess performance in the escape-optimal conditions, and it was calculated by dividing the number of center-key responses per reinforcer by 20 . The number 20 was selected because, on average, each food presentation required 20 responses. That is, for half of all trials, 10 responses would result in a stimulus change, and not food delivery. For the other half of the trials, food would be delivered following 10 responses (i.e., the completion of an FR-10 schedule). Thus, because of the probability statement in effect for the lowest ratio requirement (p $=.5$ ), each pair of completed ratios resulted in food delivery. In the present experiment, the efficiency index should range from 1.0 (if subjects responded most efficiently on the center key, escaping as soon as a higher ratio requirement was presented), to 2.25 . The value of 2.25 is indicated because of the probability and schedule values in the current experiment. On average, each reinforcer required 45 responses (i.e., 45 is the sum of the products of each ratio value [10, $40,80,160]$ multiplied by its respective probability). If no escape responding occurred, and the obtained ratio requirements perfectly matched the prescribed probability statements, 45 responses would be required for each reinforcer delivery; dividing 45 by 20 results in an 
Table 2.

Experimental conditions for two subjects in Experiment 1 that did experience persistence-optimal conditions, by subject number. The Optimal column indicates whether escaping or persisting resulted in the lowest mean response requirement. The Signals column indicates whether or not stimulus changes accompanied ratio changes. The escape requirement (an FR value) is indicated in the Escape column. Response rate on the center key (key pecks per minute), the number of escape responses per session, food reinforcement rate (reinforcers per minute), and the efficiency index are also presented. When signals were absent, the center key remained white. Note that the escape key was not available in the first condition (i.e., initial training) and the second condition for Subject A3. The symbol ${ }^{\circ}$ denotes that sessions ended with 40 grain deliveries rather than 80 . The symbol ${ }^{\ddagger}$ indicates that the condition was not run to stability because of concerns that extinction of the escape-key response may have occurred. To foster the recovery of this response, this condition was conducted briefly. This symbol indicates that modified stability criteria (e.g., 20\% variance from the grand mean, or fewer than 10 escape responses per session) were used.

\begin{tabular}{|c|c|c|c|c|c|c|c|c|}
\hline \multirow[b]{2}{*}{ Subject } & \multirow[b]{2}{*}{ Optimal } & \multicolumn{2}{|r|}{ Escape } & \multicolumn{2}{|l|}{ Sessions to } & \multicolumn{3}{|c|}{ Stable } \\
\hline & & Signals & Ratio & Stability & Response Rate & Escapes & Sr Rate & Efficiency \\
\hline \multirow[t]{10}{*}{ A3 } & Escape (Initial Training) & Present & $\mathrm{N} / \mathrm{A}$ & 40 & -- & -- & 1.97 & 2.28 \\
\hline & Persistence Optimal & Present & FR 1 & 16 & 124.99 & -- & 3.87 & -- \\
\hline & Persist Op, Sig & Present & FR 1 & 11 & 104.37 & 3.78 & 4.30 & -- \\
\hline & Persist Op, Unsig & Absent & FR 1 & 10 & 111.54 & 0.78 & 4.77 & -- \\
\hline & Persist Op, Sig & Present & FR 1 & 12 & 124.44 & 1.78 & 5.04 & -- \\
\hline & Escape & Present & FR 1 & 27 & 92.98 & 83.89 & 5.17 & 1.06 \\
\hline & Escape & Absent & FR 1 & 28 & 86.63 & 163.11 & 3.00 & 1.63 \\
\hline & Escape & Present & FR 1 & 9 & 93.72 & 90.67 & 5.10 & 1.08 \\
\hline & Escape & Present & FR 5 & 10 & 75.05 & 87.67 & 4.04 & 1.06 \\
\hline & Escape & Absent & FR 5 & 20 & 78.84 & 97.78 & 2.87 & 1.52 \\
\hline
\end{tabular}




\begin{tabular}{|c|c|c|c|c|c|c|c|c|}
\hline & Escape & Present & FR 5 & 13 & 81.79 & 80.44 & 4.68 & 1.01 \\
\hline & Escape & Present & FR 10 & 32 & 69.27 & 77.89 & 3.95 & 1.00 \\
\hline & Escape & Absent & FR 10 & 16 & 77.28 & 76.78 & 2.69 & 1.55 \\
\hline & Escape & Present & FR 15 & 16 & 70.39 & 65.11 & 3.15 & 1.25 \\
\hline & Escape & Absent & FR 15 & 55 & 59.03 & 42.50 & 1.73 & 1.83 \\
\hline & Escape ${ }^{\star}$ & Absent & FR 25 & 30 & 59.46 & 0.50 & 1.45 & 2.22 \\
\hline & Escape ${ }^{\star}$ & Present & FR 25 & 11 & 90.72 & 0.11 & 2.30 & 2.18 \\
\hline & Escape & Present & FR 1 & 11 & 93.86 & 76.88 & 5.78 & 1.00 \\
\hline & Escape & Present & FR 15 & 17 & 86.54 & 30.44 & 3.31 & 1.64 \\
\hline B2 & Escape (Initial Training) & Present & $\mathrm{N} / \mathrm{A}$ & 9 & -- & -- & $\overline{--}$ & 2.16 \\
\hline & Persistence & Present & $\mathrm{N} / \mathrm{A}$ & 43 & 124.37 & -- & 4.32 & -- \\
\hline & Persistence & Present & FR 1 & 20 & 102.69 & 7.56 & 4.07 & -- \\
\hline & Persistence $\star$ & Absent & FR 1 & 10 & 98.59 & 0.44 & 4.12 & -- \\
\hline & Persistence & Present & FR 1 & 47 & 100.64 & 22.22 & 3.90 & -- \\
\hline & Escape & Present & FR1 & 25 & 83.04 & 87.56 & 4.85 & 1.08 \\
\hline & Escape & Absent & FR 1 & 49 & 108.27 & 87.11 & 3.9 & 1.67 \\
\hline & Escape $^{\circ}$ & Absent & FR 1 & 21 & 99.12 & 48.11 & 3.88 & 1.56 \\
\hline & Escape $^{\circ}$ & Absent & FR 5 & 22 & 82.43 & 34.22 & 2.75 & 1.73 \\
\hline & Escape $^{\star \star}$ & Absent & FR 15 & 13 & 104.78 & 0.22 & 2.68 & 2.22 \\
\hline
\end{tabular}




\begin{tabular}{llllllll} 
Escape $^{\text {ot }^{*}}$ & Present & FR 15 & 3 & -- & -- & -- & - \\
Escape $^{\circ}$ & Present & FR 5 & 11 & 68.64 & 40.11 & 3.28 & 1.32 \\
Escape $^{\circ}$ & Present & FR 15 & 35 & 70.17 & 18.11 & 2.50 & 1.60 \\
Escape $^{\circ}$ & Present & FR 1 & 20 & 91.95 & 40.22 & 5.92 & 1.01 \\
\hline
\end{tabular}

Table 3.

Experimental conditions for the two subjects that did not experience persistence-optimal conditions in Experiment 1, by subject. The Signals column indicates whether or not stimulus changes accompanied ratio changes. When signals were absent, the center key remained white. The escape requirement (an FR) is indicated in the Escape column. Response rate on the food key (key pecks per minute), the number of escape responses per session, food reinforcer rate (reinforcers per minute), and the efficiency index are also presented. Note that the escape key was not available in the first condition (i.e., initial training). This symbol * indicates that modified stability criteria (e.g., 20\% variance from the grand mean, or fewer than 10 escape responses per session) were used.

\begin{tabular}{|c|c|c|c|c|c|c|c|}
\hline & & & Sessions to & Response & & & \\
\hline Subject & Signals & Escape Ratio & Stability & Rate & Escapes & Stable Sr Rate & Efficiency \\
\hline \multirow[t]{3}{*}{$\overline{A 4}$} & Present (Initial Training) & $\mathrm{N} / \mathrm{A}$ & 14 & 138.76 & -- & 3.10 & 2.16 \\
\hline & Present & FR 1 & 31 & 59.51 & 81.67 & 3.43 & 0.98 \\
\hline & Absent & FR 1 & 25 & 108.41 & 50.11 & 3.44 & 1.80 \\
\hline \multirow[t]{3}{*}{ B1 } & Present (Initial Training) & $\mathrm{N} / \mathrm{A}$ & 28 & 118.02 & -- & 2.74 & 2.22 \\
\hline & Present & FR 1 & 20 & 102.02 & 79.22 & 6.24 & 0.97 \\
\hline & Absent & FR 1 & 19 & 99.73 & 89.94 & 3.64 & 1.64 \\
\hline
\end{tabular}




\begin{tabular}{|c|c|c|c|c|c|c|}
\hline Present & FR 5 & 39 & 76.00 & 74.18 & 4.50 & 1.01 \\
\hline Absent & FR 5 & 51 & 108.86 & 70.33 & 3.87 & 1.73 \\
\hline Present & FR 5 & 11 & 87.10 & 89.67 & 5.34 & 1.05 \\
\hline Present & FR 10 & 10 & 78.75 & 84.56 & 4.73 & 1.04 \\
\hline Absent & FR 10 & 69 & 71.83 & 65.00 & 2.54 & 1.62 \\
\hline Present & FR 10 & 26 & 66.95 & 82.67 & 3.91 & 1.03 \\
\hline Present & FR 20 & 25 & 42.75 & 85.42 & 2.31 & 1.04 \\
\hline Absent $\star$ & FR 20 & 22 & 94.43 & 1.56 & 2.38 & 2.23 \\
\hline Present & FR 20 & 17 & 82.16 & 28.89 & 2.72 & 1.69 \\
\hline Present & FR 1 & 40 & 88.92 & 86.11 & 5.44 & 1.03 \\
\hline
\end{tabular}


efficiency index value of 2.25 .

The patterning of escape responses was examined graphically in two ways. First, the number of escape responses was analyzed as a function of the ratio value in effect when the responses occurred. If responding was completely efficient, an escape response would occur as soon as the lowest ratio was completed, in the absence of food delivery (i.e., escapes should occur during the second ratio). As a complementary analysis, the number of reinforcers that were delivered upon the completion of each ratio was also examined. As persistence (i.e., sunk-cost behavior) increases, the number of reinforcers obtained from the larger ratio values should also increase.

Results

\section{Initial training.}

All subjects pecked the center key during the first session of initial (FR) training so no remedial key-peck training was required. For two subjects, stable response rates during initial training were 118 and 139 key pecks per minute (subjects B1 and A4, respectively). Under these conditions, mean reinforcer rates of 2.74 (B1) to 3.10 (A4) reinforcers per minute and mean efficiency indices of 2.22 (B1) and 2.16 (A4) were obtained. These data are presented in the first line for each subject in Table 3, and as the first bars (labeled initial), in Figures 2 (response rate), 3 (reinforcer rate), and 4 (efficiency index).

For the other two subjects (A3, B2), initial training was not continued until stable responding was observed; these data are presented in the first line for each subject in Table 2.For one of these subjects (A3), 40 sessions were conducted before advancing to the next condition, and the condition was changed based on a 40-session fixed-time stability criterion. For the final nine sessions, A3 exhibited a mean response rate of approximately 90 key pecks per minute 
(range: 50 - 109), a mean reinforcement rate of 1.97 reinforcers per minute (range: $1.01-2.91$ ), and a mean efficiency index value of 2.28 (range: $1.78-3.00$ ). For subject B2, nine sessions (instead of 10) were conducted before advancing to the next condition due to experimenter error. The data from the final 8 sessions fell within the range of the mathematical stability criteria. For the final 8 sessions, a response rate of 90 responses per minute (range: 69 -99), a reinforcer rate of 2.11 reinforcers per minute (range: $1.82-2.55$ ), and an efficiency index of 2.16 (range: 1.81 2.71) were obtained. Thus, the data obtained from subjects $\mathrm{A} 3$ and $\mathrm{B} 2$ were consistent with the data from subjects B1 and A4, despite the application of different stability criteria (cf. the first line per subject of Tables 2 and 3; the first bar of Figures 2, 3, and 4, for response rate, efficiency index, and reinforcement rate, respectively).

\section{Experimental manipulations.}

Two subjects began with persistence-optimal conditions and two began with escapeoptimal conditions. Tables 2 and 3 contain the order of the experimental conditions and the number of days for which each condition was in effect. These tables also contain information on the average stable response rate, the average number of escape responses, and efficiency index values. Averages presented in this table are based on the final nine (stable) sessions in each condition.

For one subject (B2), it became necessary to terminate sessions after 40 (rather than 80 ) grain presentations. This change was made because this subject gained so much weight during experimental sessions that sessions could not be conducted on consecutive days. The conditions in which 40 reinforcers were delivered are noted in Table 2. 
Figure 2.

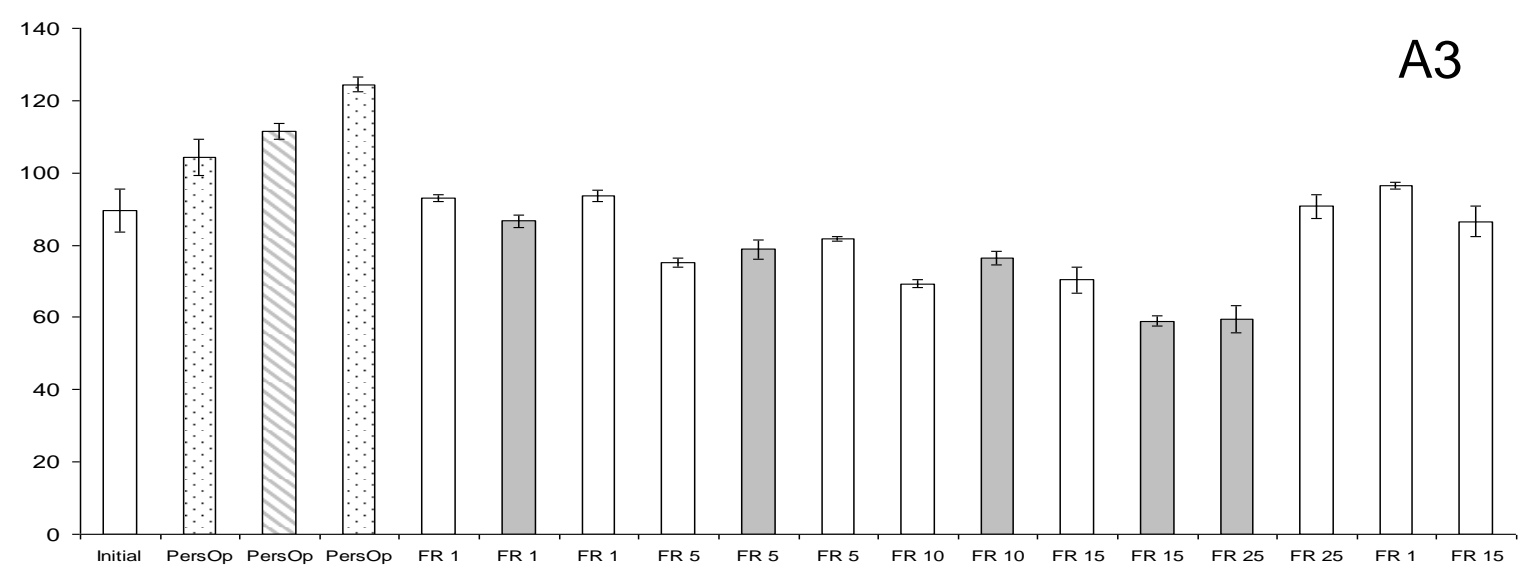

B2
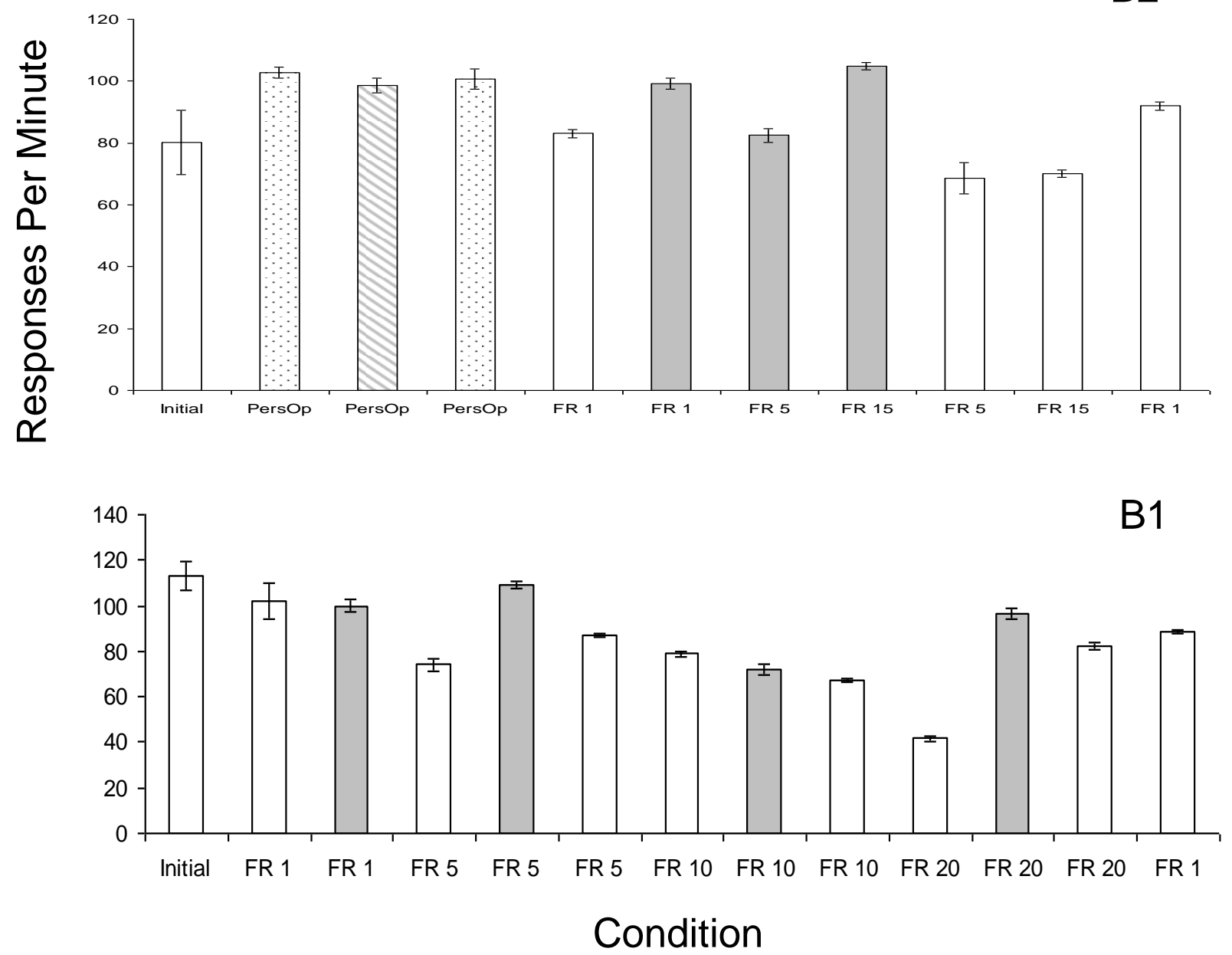
Figure 3.

A3
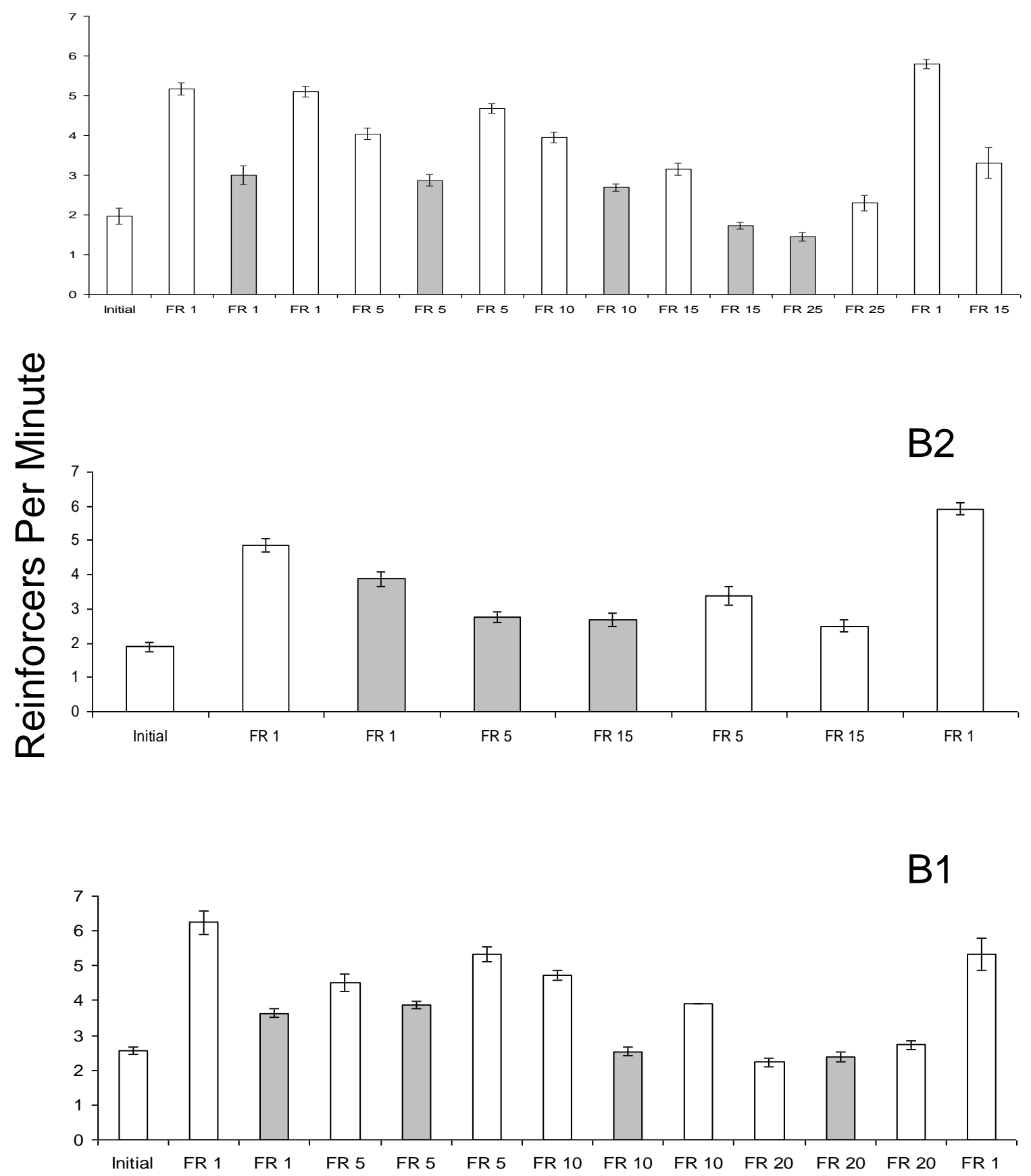

Condition 
Figure 4.
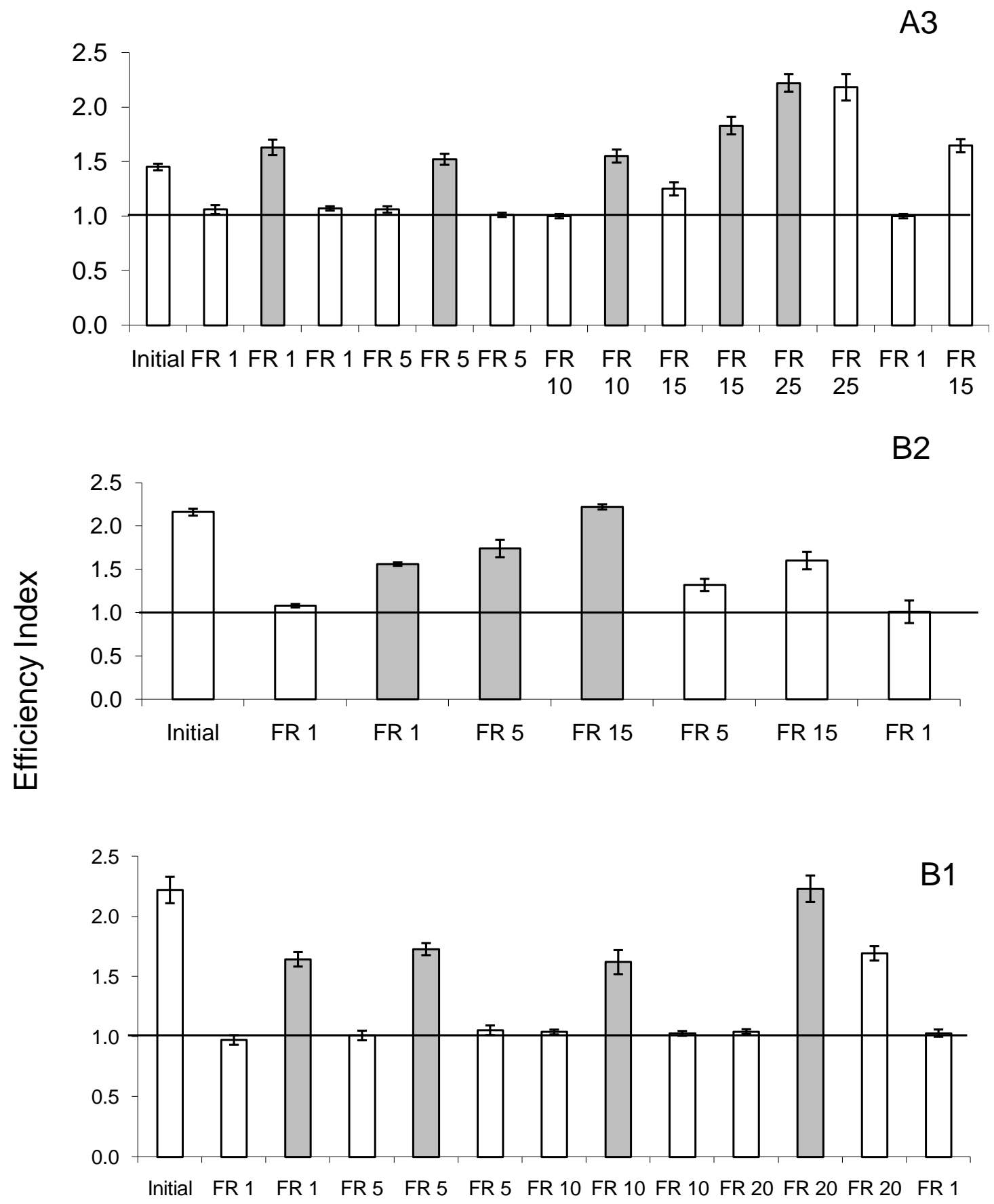

Condition 


\section{Persistence-optimal conditions.}

Throughout the persistence-optimal conditions, both subjects (A3 and B2) responded on the escape key relatively few times. In the first, signaled condition, the center-key response rate was high for subject A3 (104 key pecks per minute; see Figure 2 and Table 2), and this subject escaped an average of 3.78 times per session (see Figure 5 and Table 2). The response rate on the center key was approximately the same for subject B2 (103 key pecks per minute; see Figure 2 and Table 2), and B2 emitted a larger absolute number of escape responses (7.56 per session; see Figure 3 and Table 2) than subject A3. For each Figure mentioned above, dotted bars indicate persistence-optimal conditions with signals, and striped bars represent persistence-optimal conditions without signals.

The removal and reintroduction of the signals (i.e., changing key colors) did not systematically change either the center-key or escape-key response rates for subjects A3 and B2 (see the first three bars of the top and middle panels of Figures 2 and 5, respectively). However, for subject A3, a large difference was observed between the center-key response rates in the two signaled conditions, with a higher response rate in the second condition; see the third through fifth rows of Table 2. Thus, for this subject, the history with the unsignalled condition may have influenced responding in the signaled condition.

Escape data from the persistence-optimal conditions for subject A3 are presented in the first three bars of the top panel of Figure 5. In all three conditions, fewer than four escape responses per session occurred. Escape data for the persistence-optimal condition for subject B2 are presented in the first three bars of middle panel of Figure 5. For this subject, the removal of the signals resulted in a decrease in the number of escape responses (mean $=0.44$ escapes per session, down from 7.56 in the initial condition), and the return of the signals was accompanied 
by an increase in escape responding (mean $=22.22$ escapes per session). As with the response rate for subject $\mathrm{A} 3$, the reversal to the signaled condition resulted in an increase in escape responding by subject $\mathrm{B} 2$.

Taken together, the data from these subjects suggest that, under persistence-optimal conditions as described by Navarro and Fantino (2005), subjects consistently persisted, escaping only a few times. The presence and absence of signals affected rates of responding and escaping differently for these two subjects. The rate of escaping changed across conditions for subject B2, but not for subject A3. The center-key response rate changed across conditions for subject A3, but not for subject B2. For both subjects, changes were observed between the first and second signaled conditions for one of the two primary dependent measures (center-key responses per minute or escape-key responding), but neither subject had changes in both measures.

Escape-optimal conditions. The two subjects that experienced the persistence-optimal conditions (A3, B2) were then exposed to the escape-optimal procedure, which was the initial condition for the other two subjects. The order of the experimental conditions experienced by the subjects not participating in the persistence-optimal conditions (B1, A4) is presented in Table 3.

Subject A4 died during the replication of the signaled condition with the FR 1. Although data from initial conditions are included in the table, they will be excluded from subsequent analyses because the reversal was not completed. Results from the three remaining subjects (A3, B2, B1) will be discussed together.

Center-key responding. Center-key response rates across conditions are presented in Tables 2 and 3 and in Figure 2. In Figure 2, for the escape-optimal conditions, the ratio requirement on the escape key is presented in the labels. As in all of the figures to be discussed, white bars indicate that signals were present, and gray bars indicate the absence of signals. 
Figure 5.

A3

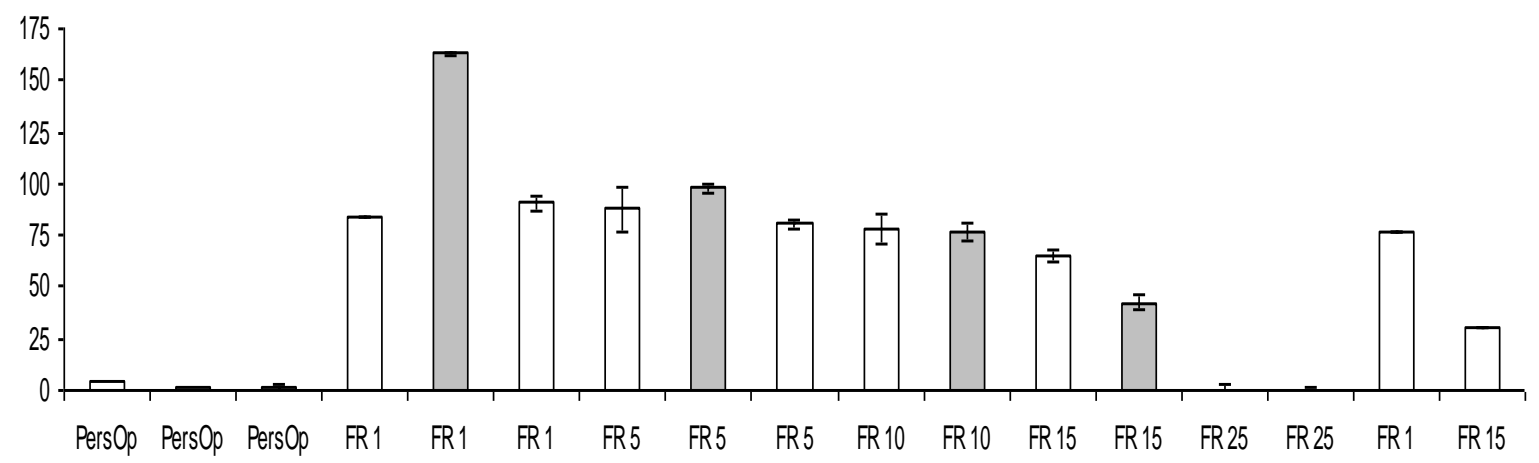

B2
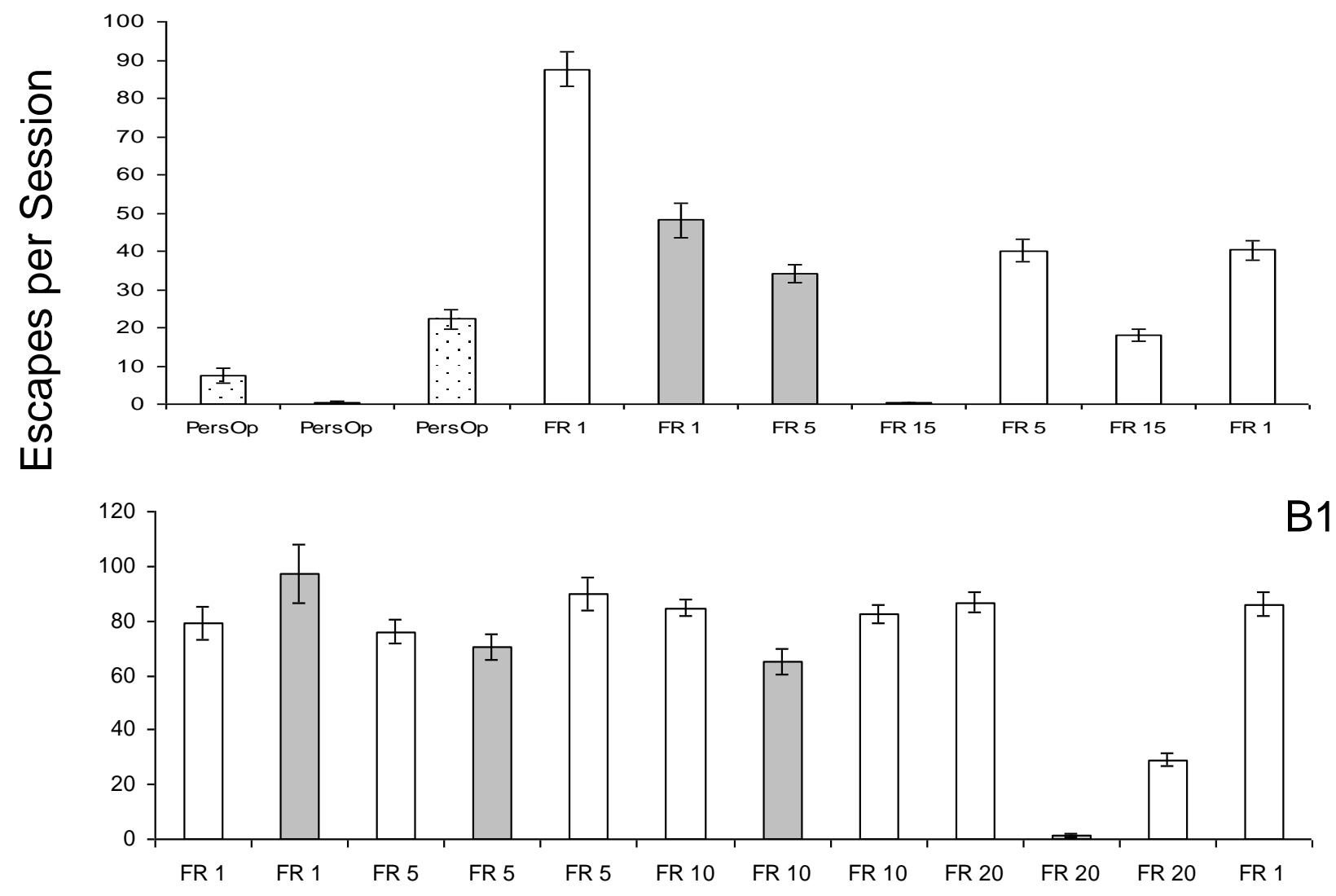

Condition 
Data presented in this figure (and in the other figures of this experiment) are from the final 9 stable session. Error bars represent the standard error of the mean $( \pm \mathrm{SEM})$.

In the signaled conditions, the center-key response rate generally decreased as a function of the increase in the escape-key requirement. For subject A3, mean response rates of 93, 75 and 69 key pecks per minute were obtained in the FR-1, FR-5, and FR-10 signaled conditions, respectively; see the top panel of Figure 2. For subject B2, the mean response rates were 83, and 69, key pecks per minute in the FR-1 and FR-5 signaled conditions, respectively; see the middle panel of Figure 2. For subject B1, the mean response rates were 102, 76, and 43 key pecks per minute in the FR-1, FR-5, and FR-20 signaled conditions, respectively; see the bottom panel of Figure 2. Although the general pattern was of a decrease, there were some exceptions. For example, for subject A3, center-key response rates were approximately equivalent when the escape requirement was FR 10 (69 key pecks per minute) and FR 15 (70 key pecks per minute), and subject B2 had approximately the same response rate when the escape requirement was FR 15 (70 responses per minute) as when it was FR 5 (69 responses per minute).

The removal of the signals did not systematically affect center-key response rates (Figure 2, gray bars). For some conditions, increases in response rate occurred in the absence of the signals, but this change was not ubiquitous. For subject A3, when the escape response requirement was a single response or 10 responses, there was an increase in escape responding in the unsignalled conditions relative to the signaled conditions. For subject B2, only the FR-1 conditions could be compared because of the increasing escape requirement in the unsignalled conditions; the response rate in the signaled condition (102 key pecks per minute) was slightly higher than the response rate in the unsignalled condition (98 key pecks per minute), but the magnitude of the difference was small. For subject B3, higher response rates in signaled 
conditions relative to the unsignalled conditions were observed when the escape requirement was an FR 10, but higher response rates were observed in unsignalled conditions (relative to signaled conditions) when the FR 5 and FR 20 were in effect. In summary, removing the signals failed systematically affect center-key response rates.

When the signals were reintroduced following the unsignalled condition, the center-key response rates generally did not return to their initial levels. For example, for subject A3, centerkey response rates were not recovered in the FR-5 conditions, with higher response rates in the second signaled condition (81 key pecks per minute) than in the first (75 key pecks per minute). The same pattern was observed for subject B1 in the FR-1 and FR-20 conditions, but a decrease was observed in the FR-10 condition. Because consecutive reversals were not conducted for B2, data from this subject were excluded from this analysis. Thus, the experience with the unsignalled condition failed to produce a replication of the response rate in the signaled conditions in most cases.

Center-key reinforcement. The center-key reinforcement rate, in food presentations per minute, is presented in Figure 3. In this figure, white bars indicate signaled escape-optimal conditions, and gray bars indicate unsignalled escape-optimal conditions. The first bar for each subject represents the initial condition in which the escape key was not available. For all subjects, the reinforcement rate decreased in the unsignalled conditions (gray bars) relative to the signaled conditions (white bars). For example, subject A3 earned 5.17 reinforcers per minute in the first signaled FR-1 condition, but only 3.00 reinforcers per minute in the unsignalled FR 1 condition. When the FR-5 escape requirement was in place, 4.04 reinforcers per minute were obtained in the signaled condition, and 2.87 reinforcers per minute were obtained in the unsignalled condition. This decreasing pattern was consistently observed across subjects and FR 
values. That is, for several conditions, decreases in reinforcement rate were observed in the absence of signals (cf., subject A3, FR 1, 5, and 10; subject B1, FR 1 and 10). This decrease indicates a failure to maximize reinforcement, and, therefore, inefficient responding. This finding was supported by the efficiency index, as well (see below).

As a complementary analysis of reinforcer delivery, Figure 6 contains stacked bar graphs depicting the distribution of reinforcers across ratios. In this figure, white bars indicate escapes that occurred when the lowest ratio was in effect, gray bars indicate escapes that occurred when the second ratio was in effect, and striped bars indicate escapes that occurred during the third or fourth ratios. Labels indicate whether signals were present or absent (Sig/Unsig) and the ratio requirement on the escape key (e.g., FR 25). In general, most reinforcer deliveries occurred following the completion of the first ratio (VR 10, white bars). For example, for subject A3, $81 \%$ of reinforcers were delivered following the first ratio, across all conditions. In the signaled conditions, $84 \%$ of reinforcers were delivered following the completion of the first ratio, and $13 \%$ were delivered following completion of the second ratio (gray bars). Across the unsignalled conditions, $74 \%$ of reinforcers were delivered following the first ratio and $20 \%$ were delivered following the completion of the second ratio. With increasing escape requirements, an increase in reinforcers delivered after the completion of the larger ratios was also observed. This finding is consistent with the previous assertion that the higher ratios were experienced more often (i.e., fewer escapes occurred when lower ratio requirements were in effect; see Figure 6).

For each subject, there was at least one condition in which the obtained distribution of reinforcers resembled the programmed probability statements, with half of the reinforcers following the first ratio, a quarter after the second ratio, and a quarter split between the third and 
Figure 6.

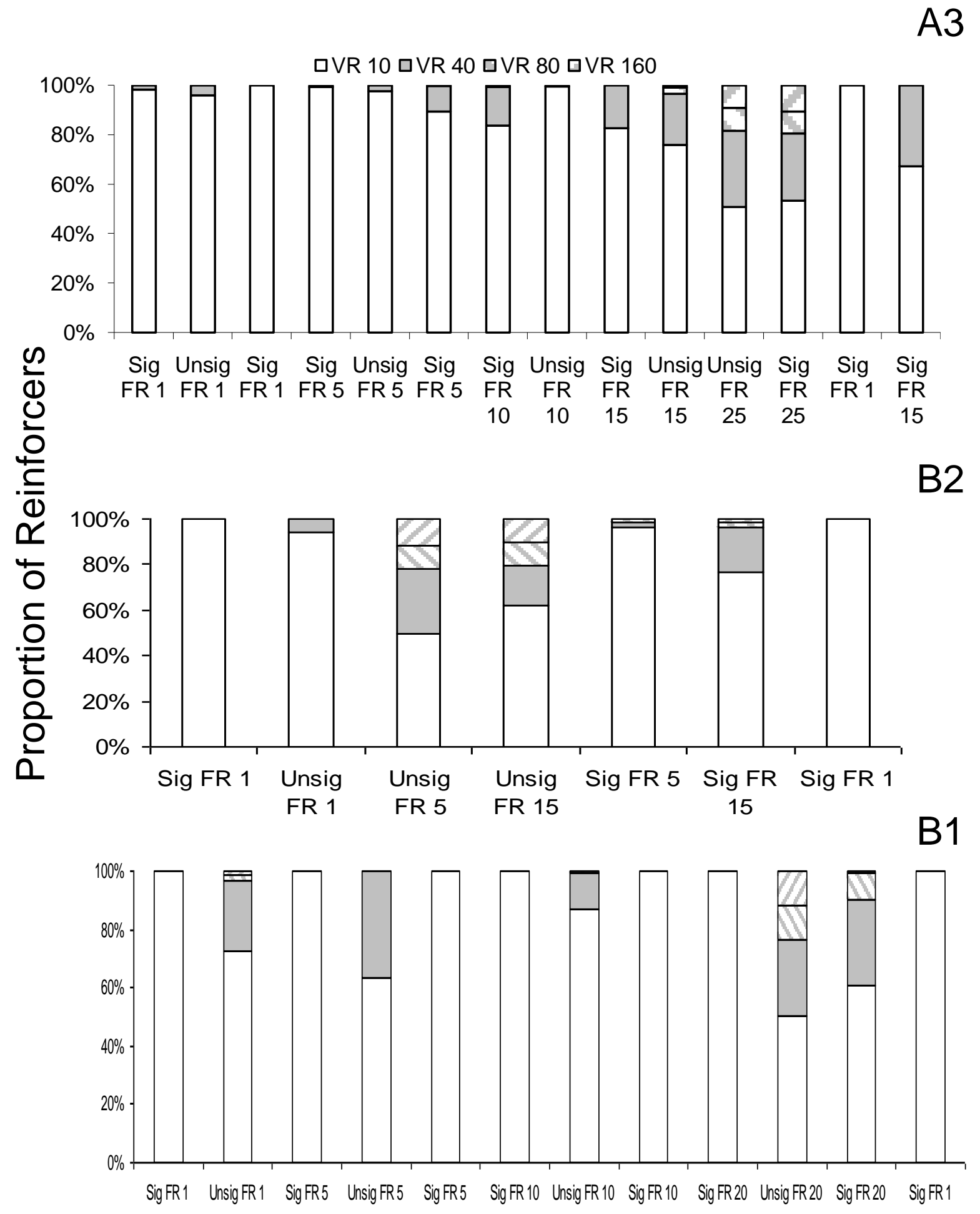

Condition 
fourth ratios. This pattern of reinforcer deliveries was observed in the unsignalled FR-20 condition for subject B1, the unsignalled FR-5 and FR-15 conditions for B2, and the signaled FR-15 condition for subject A3. Overall, then, when signals were present, most reinforcers were earned from the first ratio than when signals were absent. When the signals were absent and larger ratios were in effect on the center key, more reinforcers were earned from larger ratios than from the smaller ratios. Thus, the pattern of reinforcer delivery further supports the notion that behavior became less efficient in the absence of the signals and with increasing escape requirements. This conclusion was previously established with other dependent measures (e.g., escape responding, efficiency index), and was consistently observed across subjects.

Efficiency index. The efficiency index is a measure of persistence, and is presented (with data from the final nine stable sessions) in Figure 4. In this figure, white bars indicate signaled escape-optimal conditions, and gray bars indicate unsignalled escape-optimal conditions. The labels indicate the ratio in effect on the escape key. Error bars represent \pm SEM and the horizontal line at 1.0 indicates the most efficient pattern of responding, with 20 responses emitted per reinforcer delivery. A value of 1.0 indicates that subjects did not continue to respond following a stimulus change, i.e., engaged in perfectly efficient responding. Efficiency, here, is defined as a minimization of the number of responses emitted per food delivery. Because of the arrangement of ratio requirements and probability statements used in the current procedure, on average, escaping following the first stimulus change was the most efficient pattern of responding. Generally, when signals were present and the escape-ratio requirement was a single response, the efficiency index for all subjects was close to 1.0 (range: $0.97-1.08$ ), indicating that subjects emitted approximately 20 responses per food delivery (Figure 3, white bars).

When signals were removed (Figure 4, gray bars), the efficiency index increased, 
indicating that subjects made more than 20 key pecks per reinforcer delivery, i.e., less efficient responding. All of the 11 unsignalled conditions presented in Figure 3 had efficiency index values greater than 1.0. By contrast, 16 of the 20 signaled conditions (white bars, not initial conditions) had values at or around 1.0 (i.e., had error bars overlapping the 1.0 line; range $=0.97$ - 1.06), and the remaining 4 conditions had efficiency index values greater than 1.0 (i.e., error bars that did not intersect the 1.0 line). The signaled conditions with efficiency index values greater than 1.0 had escape-ratio requirements greater than 1 response (e.g., FR 25 for subject A3, FR 20 for subject B1, and FR 15 for B2; see also the efficiency index column of Tables 2 and 3).

A maximum efficiency index value of 2.23 was obtained for subject B1 (FR 20, unsignalled condition), and subjects A3 and B2 had maximum efficiency index values of 2.22 (FR-25 unsignalled condition, FR-15 unsignalled condition, respectively). The obtained maximum efficiency index values approached the prescribed maximum of 2.25, (i.e., 45 responses per reinforcer; see Data Analysis for explanation). This value indicates that subjects were generally persisting (rather than escaping), making more than twice as many responses as were required for each reinforcer delivery (i.e., that the pattern of persistence was inefficient). Thus, in general, the efficiency index increased when the ratio changes were unsignalled or when the response requirement to escape increased and the signals were present. An increase in the efficiency index indicates an increase in persistence and completion of ratios other than the initial, lowest value.

Escape responding. Overall, escaping was relatively consistent across the signaled conditions (see white bars in Figure 5). For the escape-optimal conditions, the ratio requirement 
on the escape key is presented in the $\mathrm{x}$-axis label. White bars indicate signaled escape-optimal conditions, and gray bars indicate unsignalled escape-optimal conditions.

Across all signaled conditions, subject A3 escaped an average of 65.90 times per session (range: 0 -108). The increased escape requirement decreased the rate of escaping only at the highest ratio values during signaled conditions (e.g., FR 15, FR 25). When the conditions with the lowest number of escapes are excluded (FR 25, FR 15), the mean increases to 80.37 escapes per session (range: 53 - 108). Similarly, an average of 76.74 escapes per session (range: $21-$ 113) was observed for subject B1, and, when the condition with the fewest escapes per session is excluded (FR 20, 28.89 escapes), the average increases to 83.57. Because there were 80 reinforcers per session, and these reinforcers were delivered upon completion of the lowest ratio for half of the trials, a value of approximately 80 escapes per session suggests that responding was relatively efficient, and this conclusion is supported by other data (see efficiency index data, presented below).

In the unsignalled conditions (Figure 5, gray bars), escaping systematically decreased as a function of the increasing response requirement. For subject A3, an average of 163.11 escapes occurred during the FR-1 condition (range: 97 - 205), 97.78 escapes occurred during the FR-5 condition (range: 66 - 129), and 76.78 escapes occurred during FR-10 condition (range: 63 90). For subject B2 (middle panel), a decrease was observed between the FR-1 condition (mean $=87.56$, range: $63-120)$ and FR-5 condition (mean $=34.22$, range: $23-43$ ), and complete suppression was observed during the unsignalled FR-15 condition (mean $=0.22$, range: $0-1)$. For subject B1, a decrease was observed between the FR-1 (mean $=97.33$, range: $48-142$ ) and FR-5 conditions (mean $=70.33$, range: $48-94$ ), with a slight decrease again in the FR-10 condition (mean $=65.00$, range: $46-88)$ and nearly complete suppression of the escape response 
in the FR-20 condition (mean $=1.56$, range: $0-6$ ). For each of the subjects, although escape responding was relatively consistent across signaled conditions, escaping in unsignalled conditions systematically decreased as a function of the escape requirement.

A second way of examining escape responding is presented in Figure 7. In this figure, the proportion of escape responses during each ratio is presented in a stacked bar graph, by condition, similar to the presentation of reinforcer deliveries in Figure 6. White bars indicate escapes that occurred when the lowest ratio was in effect, gray bars indicate escapes that occurred during the second ratio, and striped bars indicate escapes during the third or fourth ratio. Labels indicate if the signals were present (Sig) or absent (Unsig) and the programmed escape requirement (e.g., FR 5). Data presented in this figure are from the final 9 stable sessions. For subject A3 (top panel), the FR 25 unsignalled and signaled replications were excluded from this figure due to the low rates of escaping (e.g., fewer than 1 response per session, on average).

Across most conditions, the majority of escape responses occurred during the second ratio (white bars). When the signals were present, most escape responses occurred following the first stimulus change. For example, $90 \%, 96 \%$ and $92 \%$ of escapes occurred during the second ratio (i.e., after the first stimulus change) for subjects A3, B2, and B1, respectively (top, middle, and bottom panel in Figure 7). These escapes typically occurred immediately after the change in the center key color from white to yellow. Inspection of individual-session data reveal that nearly all of the escape responses during the second ratio occurred within 3 center-key responses after the stimulus change.

In the absence of the signals (i.e., when the key was always white), the number of escapes during the first ratio increased, as indicated by the gray bar. For example, for subject A3, 53\% of escapes occurred during the second ratio, with $42 \%$ of escape responses occurring during the 
Figure 7.

A3

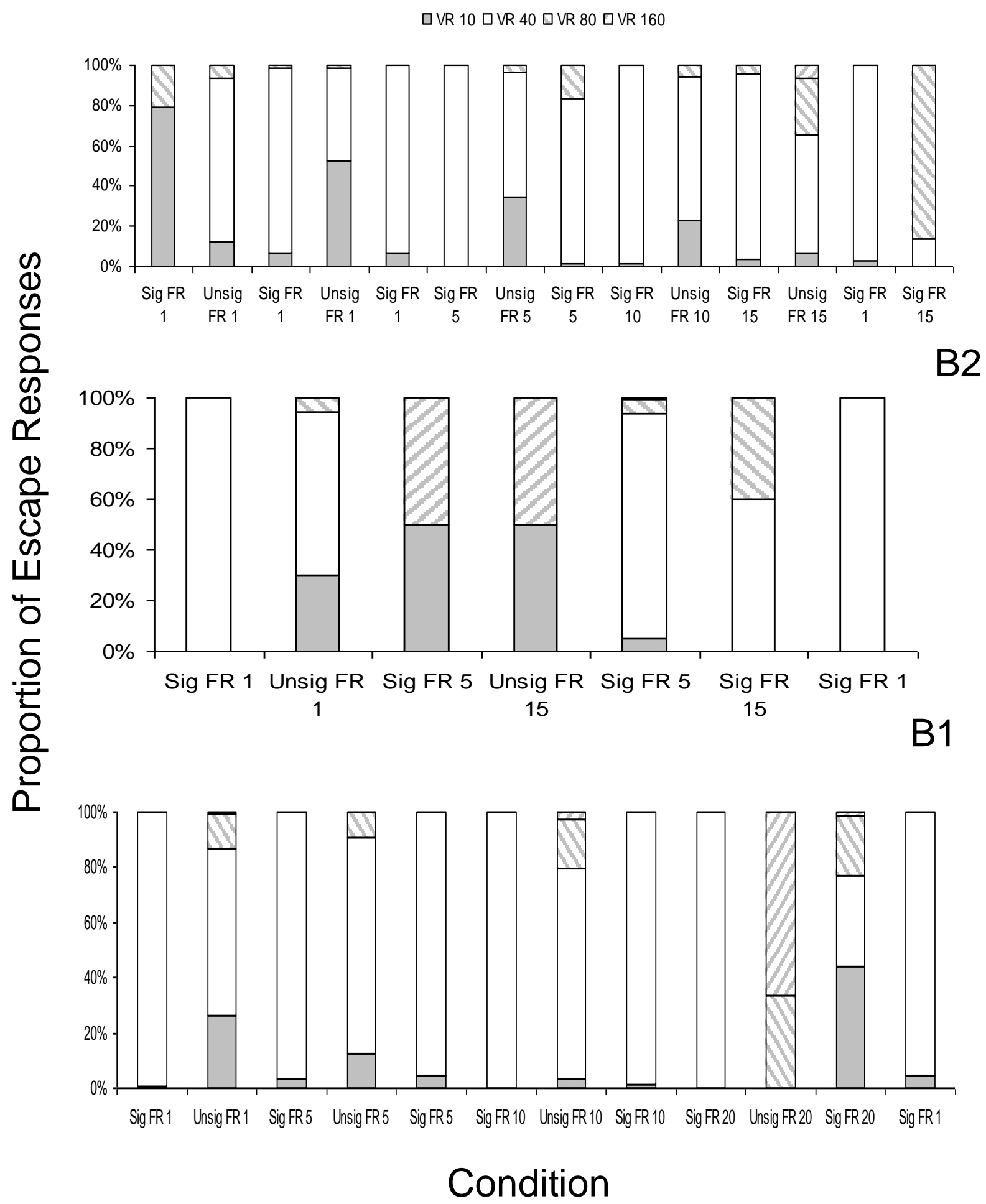


first ratio (gray bars) in the unsignalled conditions. Similar escape distributions were obtained for the other subjects. An examination of single-session data revealed that the increase of escape responses when the first ratio was in effect in unsignalled conditions was a function of a burst of responding on the escape key. That is, several escape responses were completed in succession, without intervening responses on the center key. This pattern of responding is inefficient, as escape responses likely occurred on trials during which the lowest ratio was in effect. Thus, the subjects failed to receive the food after completing a relatively small ratio.

As the escape ratio value increased, escape responses became more likely during the third or fourth ratio, in both signaled and unsignalled conditions (see left- and right-hatched bars in Figure 7). For subject A3, in the unsignalled FR-15 condition, 29\% of escapes occurred during the third ratio, and in the signaled FR-15 replication, $86 \%$ of escapes occurred during the third ratio. A similar increase in higher-ratio escapes was observed for the other two subjects. The increase in the number of escapes during the higher ratios reflects an increase in overall persistence.

Condition reversal. In a final experimental condition, the ratio requirement on the escape key was returned to an FR 1. With this reversal of response requirements, initial levels of response rate, escaping, reinforcement rate, and efficiency index were generally recovered. This can be verified by comparing the first and the last signaled FR-1 conditions. For example, for subject A3, center-key response rates were 93 key pecks per minute in both the first and last condition, the number of escape responses were similar (83.89 and 76.88 escape responses per session), and approximately five reinforcers per minute were delivered during the final nine sessions of both of these conditions. Similar replications were observed for the other subjects. Thus, the replication of initial behavioral levels was generally achieved. 
History comparison. One purpose of this study was to see if a history in persistenceoptimal conditions affected performance in escape-optimal conditions. To assess between-group differences, response rates and the number of escape responses in the first two escape-optimal conditions (signaled, unsignalled) were compared between the subjects that began with escapeoptimal conditions (B2 and A3) and the subjects that began with persistence-optimal conditions (A4 and B1). When the signals were present, the average response rate for the subjects with the history of persistence-optimal conditions was approximately 88 key pecks per minutes; 81 key pecks per minute were made by the subjects without the history of persisting. The mean number of escape responses made by the subjects with the history of persisting was $85.73 ; 80.45$ escape responses per session were made by the subjects that did not have the history of persisting. When the signals were absent, higher escape rates were observed from the subjects that did not experience the persistence-optimal conditions (mean $=125$ key pecks per minute) than the subjects with a history with persistence-optimal conditions (mean $=73$ key pecks per minute). Response rates on the center key were similar between the groups (97 and 104 key pecks per minute, with and without the history of persisting, respectively). Thus, the only large difference that was observed between the groups was in escaping in the unsignalled conditions. In these conditions, the subjects with a history of persisting escaped less than the subjects without this history.

\section{Discussion}

In the present experiment, two subjects experienced conditions similar to the persistenceoptimal conditions devised by Navarro and Fantino (2005). When persistence-optimal schedules of reinforcement were in effect, behavior was generally optimal (i.e., persistence occurred). The rate of escape responding decreased when the signals were removed, which is consistent with the 
increased persistence observed by Navarro and Fantino. Therefore, the persistence-optimal component of Navarro and Fantino's study was successfully replicated.

When presented with contingencies similar to the escape-optimal conditions (as described by Navarro and Fantino, 2005) all subjects, regardless of their initial experimental history, escaped frequently in the presence of the signals and when the escape response requirement was low, consistent with the findings of Navarro and Fantino (2005). In their first experiment, Navarro and Fantino found that subjects escaped nearly $100 \%$ of the time in the presence of the stimulus changes and, for three of the four subjects, nearly $100 \%$ of the time in their absence. Under initial conditions in the present study, for one subject (A3) the presence and absence of the signals did not significantly alter escaping, but, for a second subject (B2) the signals did differentially affect escaping. Although histories of responding in similar situations have been invoked as an important variable in the sunk-cost effect (e.g., Goltz, 1999; Navarro and Fantino, 2005; Pompilio \& Kacelnik, 2005), this was not a salient determinant of escape responding in the signaled conditions of the present experiment. That is, similar patterns of escaping were observed in the signaled conditions, but differences emerged when the signals were removed. The emergent differences suggest an interactive effect of history and the signals.

In both the present study and the work by Navarro and Fantino, escape responses occurred in signaled conditions, but conflicting results were obtained in unsignalled conditions. In the present study, when the signals were removed, subjects without a history of persisting escaped more than the subjects with the history of persistence. It is possible that, with a longer history with persistence-optimal conditions, the differences between the groups would have manifested in the signaled conditions. It is also possible that this effect may have washed out with extended exposure to escape-optimal conditions. The different effects of signals on 
behavioral persistence within the present experiment may suggest a role for individual differences in the present experiment. Although signals did affect behavioral persistence in the present experiment, additional variables that were not identified in the present study also controlled this type of persistence. In light of the conflicting results obtained for these two subjects, additional subjects should be tested in future studies, and attempts should be made to equate the experimental histories of the subjects.

Throughout the experiment, subjects were faced with the choice to continue responding or to escape and start over. During conditions when the cost was relatively low, subjects escaped. When the cost of escaping (i.e., the response requirement) increased, the frequency of escape responses decreased, ultimately, to complete suppression. The complete suppression of the escape response suggests that escaping was weakly maintained as the escape requirement increased.

In persisting and, hence, completing the center-key ratios (as indicated by the increase in larger-ratio reinforcement, see Figure 7), more responses were emitted on the center key than were required on the right key to escape. Even in the condition with the highest escape requirement programmed on the right key (FR 20), it would take an average of 10 more responses (i.e., a total of 30) to satisfy the second ratio (VR 40) on the center key after the first ratio failed to result in grain delivery. Note, too, that the VR 40 was only in effect for half of the trials in which the lowest ratio (VR 10) did not result in food. Thus, on the other half of trials, it could take an additional 70 responses on the center key to obtain food after the lowest ratio had been completed. This is a case of inefficient responding (i.e., on average, fewer responses per reinforcer were required following an escape response than persistence), governed, perhaps, by both the response requirement and the effort required of moving from the center key to the right 
key. Perhaps if the keys were physically closer together, or if another, less-effortful mechanism for escaping was employed (e.g., a treadle, available while pecking the center key), the optimal (i.e., escape) behavior could be maintained with even higher ratio requirements in effect.

The observed continuation of an established course of action is consistent with Siegel and Rachlin's (1995) notion of soft commitment, in which interrupting an existing course of action is associated with a cost. Perhaps a direct manipulation of cost in a soft-commitment paradigm would lead to conclusions similar to those obtained in the present study: with a decreased cost of interruption, there may be a point at which the soft-commitment response fails to ensure optimal (i.e., self-controlled) behavior. For example, if going to the gym is a selfcontrolled behavior, perhaps writing the workout on a calendar would be a less effective commitment response than having a friend come to your house to meet you. The writing on the calendar produces less of an interruption to ongoing behavior than having a friend arrive. If the relative cost of interruption affects the efficacy of the soft-commitment response, you may be more likely to go to the gym when a friend visits you than if you only write it on the calendar. The effective parameters of the commitment response may be an interesting avenue for future research. The failure of the less costly soft-commitment response to increase the likelihood of the optimal, self-controlled behavior would be similar to the decrease in escaping (the optimal behavior) observed with an increased effort requirement.

Increasing the likelihood of optimal responding has applied implications. For example, Leahy (2000) suggested that persisting without considering future costs (i.e., based on sunk costs) may lead to clinically significant problems. Additionally, in the investment paradigms (e.g., McCain, 1986; Strough et al., 2008), persistence is maladaptive because resources are wasted. For example, when faced with the choice to increase or decrease investment in an 
unprofitable mutual fund, when escalation is chosen, the money cannot be used for other purposes. When the investment is considered in terms of time, alternate activities cannot be pursued. By avoiding this type of maladaptive persistence, resources can be saved and quality of life may be improved.

It is possible that ratio strain may have led to the observed decreases in escape responding. That is, when the response requirement on the escape key increased, it is possible that this increase was too large for the subject to contact the change. Although not explicitly tested, data obtained from subject A3 may suggest that ratio strain was not the mechanism through which the decrease in escape responding occurred. With this subject, the response requirement was increased from 15 to 25 , and suppression was observed. After a reversal to an FR-1 condition, during which escape responding recovered, the ratio requirement was increased to FR 15. When the FR-15 escape requirement was in effect, escape responding continued. Here, the absolute magnitude of the change was four more responses than the increase from 15 to 25 . If the absolute magnitude of the ratio changes influences ratio strain, it is possible that ratio strain was not responsible for the decrease in the escape response. It is important to note, however, that there was a history of responding in the FR-15 condition which was not present for the FR-25 condition. Perhaps the history of responding in the FR-15 condition made attenuated the suppressive effects of the increasing ratio, leading to the continued escape responding in the FR15 condition that was not observed in the FR-25 condition. Building a history (i.e., adding components) with the FR-25 condition and reassessing effects of increasing ratio requirement may clarify the role of ratio strain in the present study.

Throughout Experiment 1, the obtained values of the dependent measures were largely the same throughout the signaled conditions; effects of the increasing response requirement were 
most salient in the unsignalled conditions. For example, the efficiency index increased during the unsignalled conditions relative to the signaled conditions, indicating increased persistence and less efficient responding. The degree of persistence was graded across the different escape ratio requirements, with higher ratios engendering more persistence. The graded nature of the effect suggests an interaction between the escape requirement and the signals. Additionally, the only emergent difference between the groups with different experimental histories (with persistence optimal conditions or no previous experience) was observed in unsignalled conditions. Together, these findings underscore the importance of discriminability in the sunk-cost effect, a variable implicated by several authors (cf. Bornstein \& Chapman, 1995; McCain, 1986; Navarro \& Fantino, 2005, 2007). In the present experiment, the absence of the signals was correlated with less efficient responding. Thus, in the present experiment, signals facilitated efficient responding, consistent with previous literature on the sunk-cost effect and other areas of inquiry (cf. Lieving, Reilly \& Lattal, 2006; McDevitt \& Williams, 2001; Navarro \& Fantino, 2005, 2007; Siegel \& Rachlin, 1995).

Increasing the escape response requirement is necessarily correlated with a longer delay to the onset of the next trial. That is, it takes more time to make several responses than a single response. Thus, the observed decreases in escape responding may have resulted from a combination of effort and delay. Experiment 2 was designed to separate the effects of increased effort from increased delay by explicitly manipulating the delay to the onset of the next trial following an escape response.

\section{Experiment 2}

In Experiment 1, effects of increasing response requirements were assessed in the sunkcost procedure developed by Navarro and Fantino (2005). Increasing the response requirement to 
escape resulted in a decrease in the rate of escape responding, primarily in the unsignalled conditions. The additional response requirement necessarily increased the delay to the onset of the next trial. Thus, the changes observed with increasing response requirements may have been due to the increased number of responses required, or they may have been due to the correlated increase in the delay to the onset of the next trial. Experiment 2 was designed to assess effects of increasing delay in this paradigm.

Unlike the work of Navarro and Fantino (2005, 2007) Experiment 1 used VR rather than FR schedules of reinforcement. Despite the difference in the scheduling of reinforcers, the general findings of Navarro and Fantino $(2005,2007)$ were replicated. That is, in the absence of the signals accompanying increasing response requirements, persistence was more likely to occur, relative to when the signals were present. This persistence was a form of inefficient responding, in that, on average, more responses were emitted per reinforcer than would be necessary. In the present experiment, VR schedules of reinforcement were in effect, but Navarro and Fantino used FR schedules. Thus, in order to, more directly replicate of the work of Navarro and Fantino, FR schedules were used in Experiment 2. This experiment served two primary functions: (1) to provide a comparison between FR and VR schedules of reinforcement in the sunk-cost procedure and (2) to assess effects of increasing the delay to the onset of the next trial following an escape response.

\section{Method}

\section{Subjects \& Apparatus}

Four experimentally experienced male White Carneau pigeons with largely unknown histories served as subjects in this experiment. These subjects were not the same as in Experiment 1, but they were maintained under general housing and feeding conditions identical 
to those used in Experiment 1. The same operant-conditioning chambers were also used.

\section{Procedure}

In their procedure, Navarro and Fantino (2005) used FR schedules of reinforcement and evaluated effects of the presence and absence of signals on their model of sunk cost. In the present experiment, the four subjects began with a procedure involving increasing FR schedules with stimulus changes present in a direct replication of Navarro and Fantino's work.

Initial training and the general procedure were the same as in Experiment 1. The subsequent procedures used in this experiment are diagrammed in Figure 8. Trials began with an FR 10 in effect on the center key. On half of the trials $(\mathrm{p}=.5), 3 \mathrm{~s}$ of access to grain were provided following the completion of the FR 10 schedule (dotted arrows). On one-quarter of all trials ( $\mathrm{p}=$ $.25), 3 \mathrm{~s}$ of grain access were delivered following the completion of the FR 40. Of the remaining trials, half resulted in grain access when the FR 80 was satisfied, and half when the FR 160 was satisfied. Sessions were terminated following 80 food presentations.

At any point in a trial, subjects could respond on the right (escape) key, indicated in Figure 8 by dashed lines. A single response on the escape key (FR 1) resulted in an X-s delay before the onset of the next trial, where $\mathrm{X}$ varied across conditions. The delay values used in this experiment ranged from 1 to $20 \mathrm{~s}$. At each delay value, the presence and absence of signals was manipulated. If differences were observed in response rates on the key correlated with food delivery or the number of escapes between the signaled and unsignalled conditions at a given delay value, the signals were reintroduced in a third condition with the same delay value. As in Experiment 1, some subjects (A2, B3) required sessions to be terminated following 40 grain

presentations instead of 80 , due to difficulty maintaining $80 \%$ of free-feeding body weight. The conditions in which sessions terminated with 40 reinforcers are noted in Table 4. 
Figure 8.

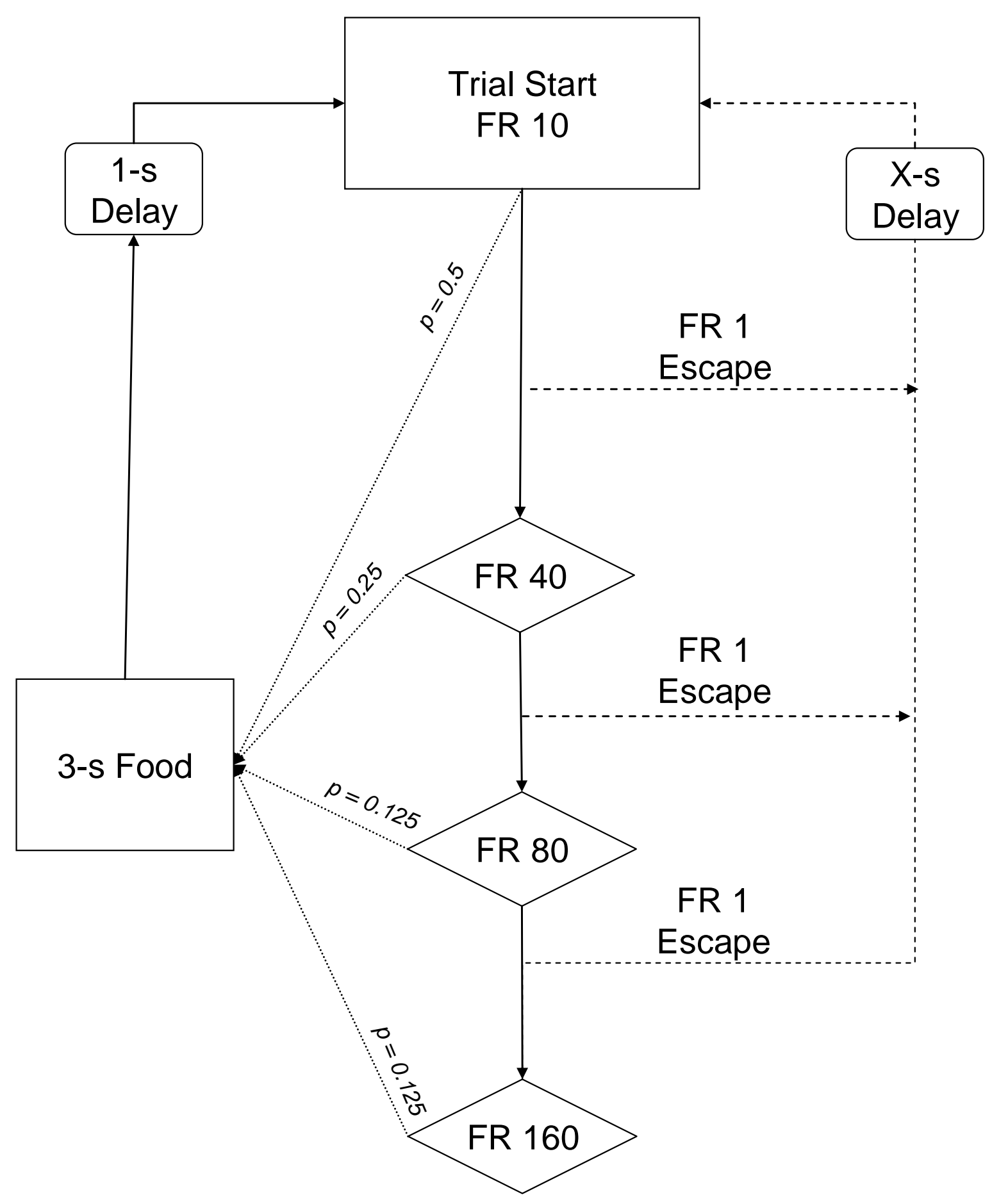


Table 4.

Conditions in Experiment 2, by subject. The Signals column indicates whether or not stimulus changes accompanied ratio changes. When signals were absent, the center key remained white. The Delay column indicates the delay between the escape response and the onset of the next trial. Note that the escape key was not available in the first condition (i.e., initial training). The Response Rate column contains the mean number of center-key pecks per minute. The rate of food reinforcement is presented in reinforcers per minute. Obtained efficiency index values are presented in the final column. The symbol ${ }^{\ddagger}$ indicates that the condition was not run to stability because the subject (due to the amount of weight gained in each experimental session) was unable to run daily. Thus, to increase the likelihood that this subject could run daily, the number of reinforcer deliveries was decreased from 80 to 40 . The symbol ${ }^{\circ}$ denotes that sessions ended with 40 grain presentations. This symbol * indicates that modified stability criteria (e.g., $20 \%$ variance from the grand mean, or fewer than 10 escape responses per session) were used.

\begin{tabular}{|c|c|c|c|c|c|c|c|}
\hline \multirow[b]{3}{*}{ Subject } & \multirow[b]{3}{*}{ Signals } & \multicolumn{3}{|c|}{ Sessions } & \multirow{2}{*}{\multicolumn{2}{|c|}{ Stable }} & \multirow[b]{3}{*}{ Efficiency } \\
\hline & & & to & Response & & & \\
\hline & & Delay & Stability & Rate & Escapes & Sr Rate & \\
\hline \multirow[t]{14}{*}{$\mathrm{A} 1$} & Present & $\mathrm{N} / \mathrm{A}$ & 20 & 69.23 & -- & 1.60 & 2.11 \\
\hline & Present & $1 \mathrm{~s}$ & 14 & 82.40 & 82.11 & 5.28 & 1.02 \\
\hline & Absent & $1 \mathrm{~s}$ & 11 & 89.96 & 107.56 & 4.08 & 1.36 \\
\hline & Present & $1 \mathrm{~s}$ & 10 & 85.27 & 86.22 & 5.05 & 1.08 \\
\hline & Present & $5 s$ & 11 & 77.87 & 83.56 & 4.07 & 1.11 \\
\hline & Absent & $5 s$ & 10 & 92.85 & 72.33 & 4.35 & 1.36 \\
\hline & Present & $5 s$ & 15 & 84.09 & 76.11 & 4.99 & 0.99 \\
\hline & Present & $10 \mathrm{~s}$ & 19 & 73.97 & 75.56 & 4.34 & 0.98 \\
\hline & Absent & $10 \mathrm{~s}$ & 28 & 77.69 & 33.67 & 2.26 & 1.91 \\
\hline & Present & $10 \mathrm{~s}$ & 16 & 76.12 & 76.00 & 4.47 & 0.99 \\
\hline & Present & $15 \mathrm{~s}$ & 41 & 78.64 & 41.00 & 2.59 & 1.67 \\
\hline & Absent & $15 \mathrm{~s}$ & 10 & 91.00 & 12.78 & 2.29 & 2.17 \\
\hline & Present & $15 \mathrm{~s}$ & 17 & 94.32 & 31.56 & 3.09 & 1.60 \\
\hline & Present $\star$ & $20 s$ & 19 & 93.27 & 25.78 & 3.13 & 1.69 \\
\hline
\end{tabular}




\begin{tabular}{|c|c|c|c|c|c|c|c|}
\hline & Absent $\star$ & $20 \mathrm{~s}$ & 16 & 95.84 & 6.67 & 2.49 & 2.08 \\
\hline & Present & $20 \mathrm{~s}$ & 43 & 102.82 & 36.22 & 3.34 & 1.69 \\
\hline & Present & $1 \mathrm{~s}$ & 12 & 96.59 & 92.78 & 5.99 & 1.07 \\
\hline \multirow[t]{11}{*}{$\mathrm{A} 2$} & Present & $\mathrm{N} / \mathrm{A}$ & 16 & 127.74 & -- & 2.88 & 2.13 \\
\hline & Present & $1 \mathrm{~s}$ & 9 & 85.73 & 86.11 & 5.13 & 1.05 \\
\hline & Absent & $1 \mathrm{~s}$ & 17 & 89.39 & 95.89 & 4.18 & 1.29 \\
\hline & Present & $1 \mathrm{~s}$ & 15 & 90.15 & 80.22 & 5.86 & 1.00 \\
\hline & Present & $5 \mathrm{~s}$ & 26 & 90.12 & 84.00 & 4.73 & 1.11 \\
\hline & Absent & $5 \mathrm{~s}$ & 14 & 86.67 & 74.89 & 4.49 & 1.13 \\
\hline & Present ${ }^{\mathrm{t}}$ & $10 \mathrm{~s}$ & 13 & 118.33 & 30.11 & 3.88 & 1.73 \\
\hline & Absent ${ }^{\star} \star$ & $10 \mathrm{~s}$ & 27 & 116.83 & 8.00 & 3.07 & 2.10 \\
\hline & Absent $^{\circ \star}$ & $15 \mathrm{~s}$ & 27 & 106.82 & 5.00 & 2.45 & 1.82 \\
\hline & Present $^{\circ} \star$ & $15 \mathrm{~s}$ & 29 & 113.97 & 3.44 & 3.13 & 2.10 \\
\hline & Present $^{\circ}$ & $1 \mathrm{~s}$ & 14 & 85.42 & 33.89 & 5.84 & 0.93 \\
\hline \multirow[t]{12}{*}{ B3 } & Present & $\mathrm{N} / \mathrm{A}$ & 22 & 70.76 & -- & 1.51 & 2.33 \\
\hline & Present & $1 \mathrm{~s}$ & 25 & 43.19 & 81.89 & 2.47 & 1.00 \\
\hline & Absent & $1 \mathrm{~s}$ & 10 & 74.37 & 57.11 & 2.38 & 1.77 \\
\hline & Present & $1 \mathrm{~s}$ & 12 & 63.55 & 82.78 & 3.78 & 1.01 \\
\hline & Present & $5 \mathrm{~s}$ & 11 & 53.88 & 75.56 & 3.04 & 0.98 \\
\hline & Absent & $5 \mathrm{~s}$ & 30 & 77.55 & 77.44 & 3.41 & 1.25 \\
\hline & Present & $5 \mathrm{~s}$ & 14 & 78.70 & 73.44 & 4.90 & 0.96 \\
\hline & Present $^{\ddagger}$ & $10 \mathrm{~s}$ & 21 & -- & -- & -- & -- \\
\hline & Present $^{\circ}$ & $10 \mathrm{~s}$ & 53 & 97.93 & 16.56 & 3.15 & 1.69 \\
\hline & Absent $^{\circ}$ & $10 \mathrm{~s}$ & 15 & 89.16 & 23.44 & 2.69 & 1.76 \\
\hline & Present $^{\circ} \star$ & $10 \mathrm{~s}$ & 21 & 88.33 & 13.78 & 3.62 & 1.37 \\
\hline & Present $^{\circ}$ & $1 \mathrm{~s}$ & 13 & 90.95 & 42.33 & 6.63 & 1.07 \\
\hline
\end{tabular}




\begin{tabular}{llllllll}
\hline B4 & Present & N/A & 16 & 130.52 & -- & 2.76 & 2.32 \\
Present & $1 \mathrm{~s}$ & 15 & 72.78 & 81.78 & 4.02 & 1.03 \\
Absent & $1 \mathrm{~s}$ & 12 & 72.69 & 140.11 & 2.74 & 1.44 \\
Present & $1 \mathrm{~s}$ & 39 & 88.87 & 78.33 & 5.21 & 1.01 \\
Present & $5 \mathrm{~s}$ & 22 & 87.66 & 83.89 & 4.99 & 1.03 \\
Absent $\star$ & $5 \mathrm{~s}$ & 42 & 108.00 & 0.56 & 2.69 & 2.27 \\
& & & & & & \\
Present & $5 \mathrm{~s}$ & 24 & 92.40 & 73.56 & 5.12 & 1.12 \\
Present & $1 \mathrm{~s}$ & 33 & 98.70 & 76.67 & 6.09 & 0.98 \\
\hline
\end{tabular}

\section{Data analysis}

Data analysis procedures were the same as in Experiment 1. Stability criteria were also the same as in Experiment 1.

\section{Results}

A list of the conditions in Experiment 2 is presented in Table 4. Additionally, Table 4 contains the number of sessions until stability was attained, the mean stable response and reinforcer rates, the number of escape responses, and the efficiency index for each condition. Initial training.

All subjects reliably responded on the first day of the experiment, so no remedial keypeck training was necessary. Center-key response rates are presented across conditions in Figure 9. White bars indicate signaled conditions, and gray bars indicate unsignalled conditions. The first bar for each subject in Figure 9 represents the initial condition in which the escape key was not available, and subsequent labels indicate the delay following an escape response. As with all figures presented in this experiment, data are from the final 9 stable sessions, and error bars represent \pm SEM. During the initial training, center-key response rates of $69,128,71$, and 131 
Figure 9.
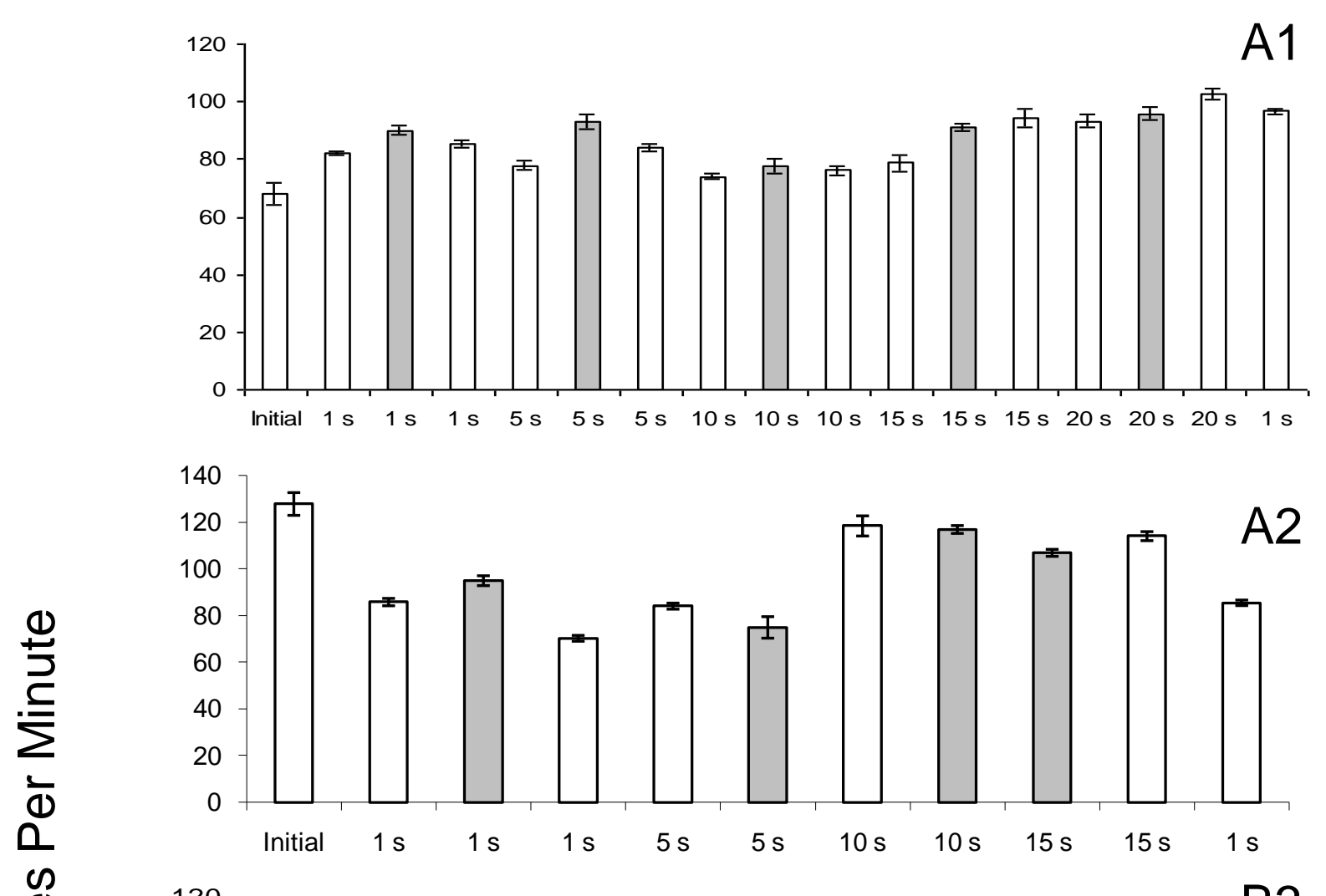

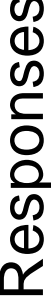
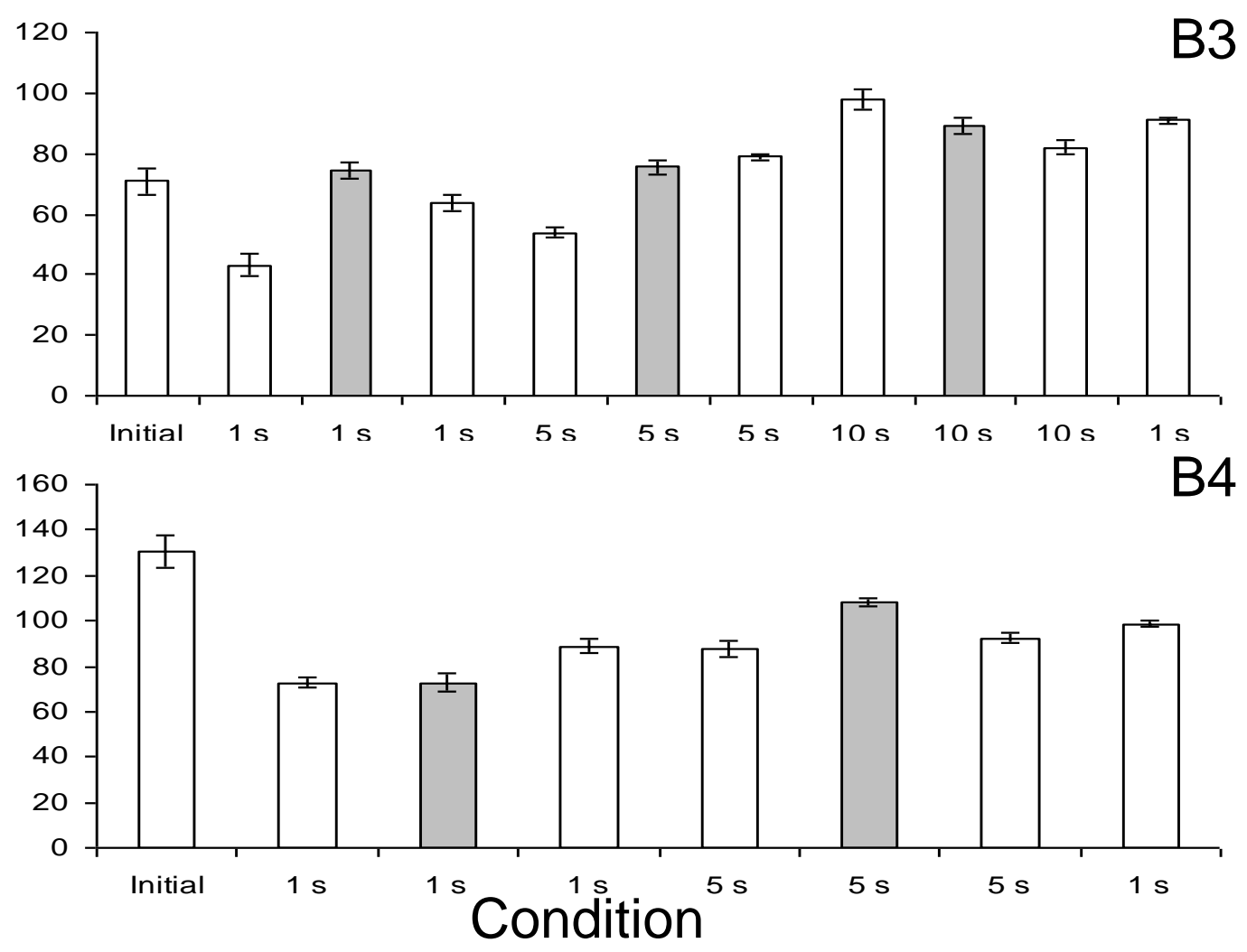
responses per minute were observed for subjects A1, A2, B3, and B4, respectively (see the first bar of Figure 9).

Efficiency index data are presented in the first bar of Figure 10. White bars indicate signaled conditions, and gray bars indicate unsignalled conditions. The horizontal line at 1.0 represents perfectly efficient responding. During the initial condition, the obtained efficiency index values were 2.11, 2.13, 2.33, and 2.32, for A1, A2, B3, and B4, respectively. Mean reinforcer rates of 1.60, 2.88, 1.51, and 2.76 food deliveries per minute were obtained, and are presented in the first bar of each panel of Figure 11, which has the same formatting as Figure 10. Experimental manipulations.

Center-key responding. Increasing delays following escape responses did not systematically affect center-key response rates in the signaled conditions (see Table 4 and Figure 9, white bars). For subject A1, increasing delays resulted in decreasing center-key response rates. For example, response rates of 82, 78, 74 keypecks per minute were obtained in the first signaled conditions with 1-s, 5-s, and 10-s delays. During the condition with the longest delays (i.e., 20 s), an increase in response rate (93 keypecks per minute) was observed relative to the previous conditions. Subject A2 had consistent response rates across the second signaled 1-s condition and the first signaled 5-s condition (90 responses per minute in both), and an increase in response rates when the delay was increased to $10 \mathrm{~s}$ and $15 \mathrm{~s}$ (118 and 114 keypecks per minute, respectively). For subject B3, increases in delay were accompanied by increases in response rate, e.g., 43 keypecks per minute in the first signaled 1-s delay condition and 98 keypecks per minute in the first 10-s delay condition; see the white bars of the third panel of Figure 9. For subject B4, response rates were generally consistent across signaled conditions, with a mean of 88 responses per minute (range: 75 -98). In sum, increasing delays did not systematically affect center-key 
Figure 10.
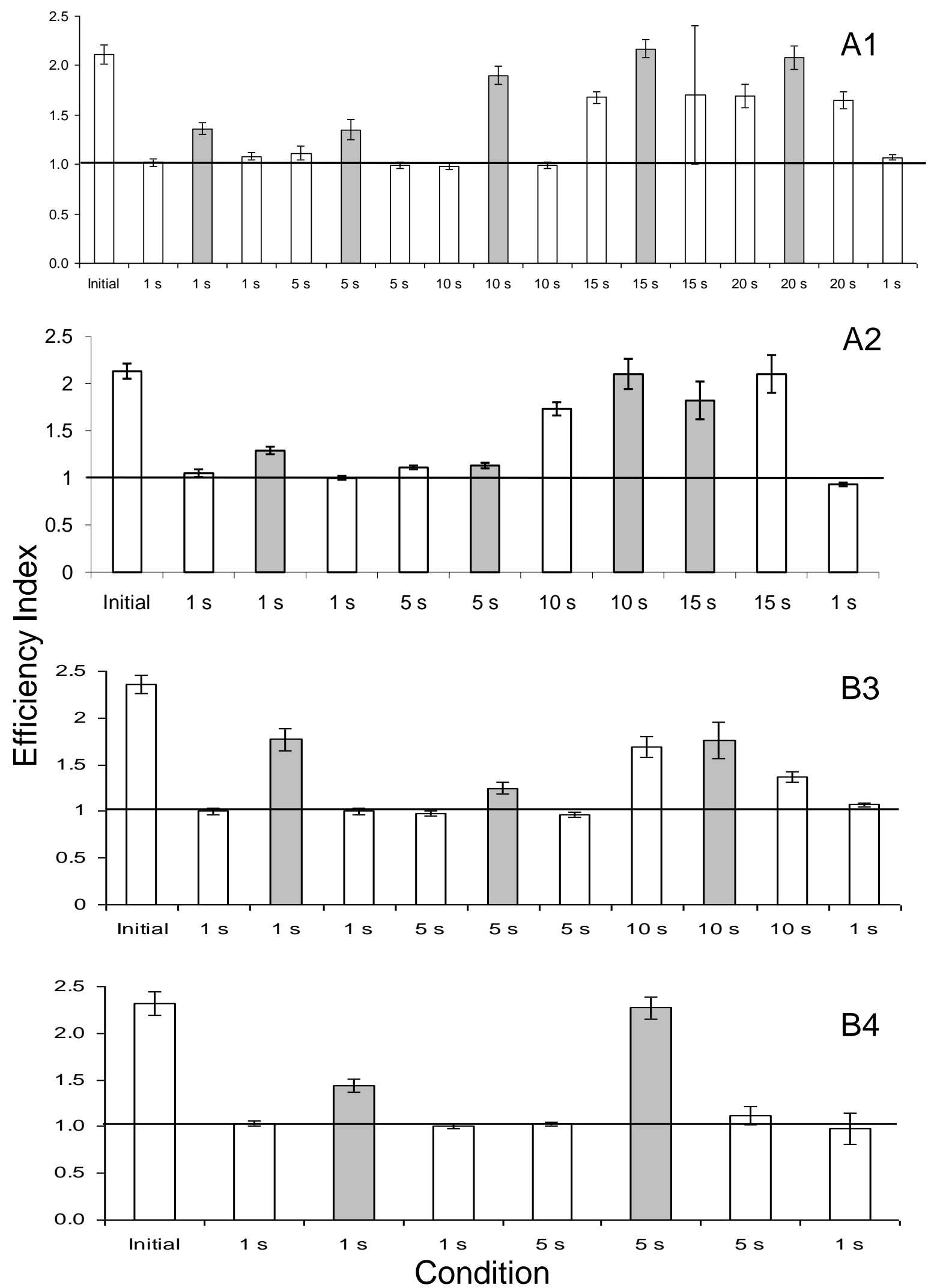
Figure 11.
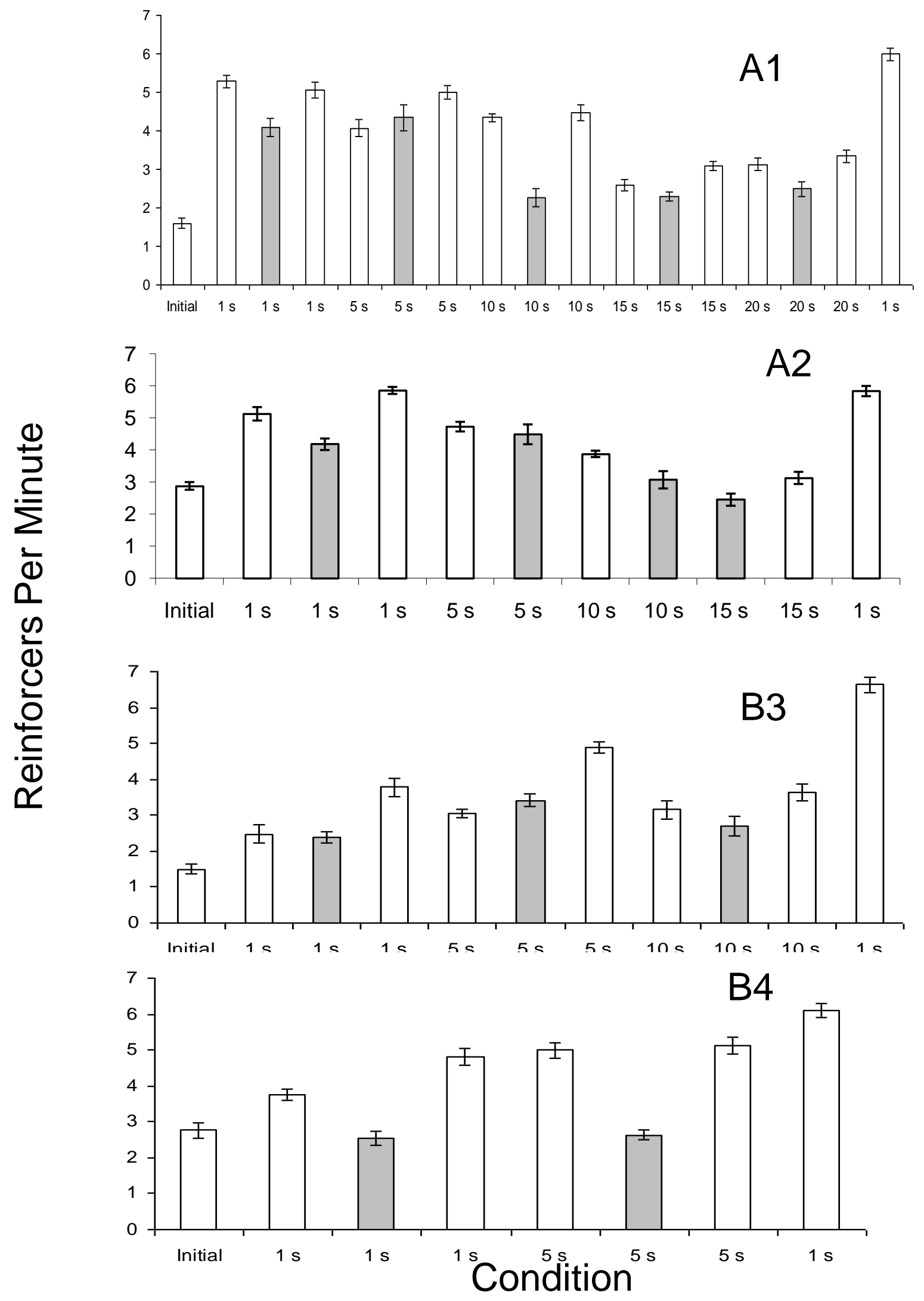
response rates, with increases for some subjects (e.g., B3) and decreases for others (e.g., A1).

Response rates on the center key were typically higher in unsignalled conditions (Figure 9, gray bars) than in signaled conditions (white bars), especially when considering the initial changes from signaled conditions to unsignalled conditions. This effect was most pronounced for subjects A1, B3, and B4 (first, third, and fourth panels). For subject A1, 82 responses per minute were observed in the 1-s, signaled condition, and, in the absence of the signals, the response rate increased to 90 key pecks per minute. Likewise, increases were observed in the absence of the signals, relative to their presence, in the 5-s conditions (78 and 93 responses per minute), the 10s conditions ( 74 and 78 responses per minute), 15-s conditions (79 and 91 responses per minute), and the 20-s conditions (93 and 96 responses per minute). Similar patterns were observed for the other subjects (e.g., 10-s delay condition for subject A2, 5-s delay condition for subjects B3 and B4). For all subjects, higher response rates occurred in the unsignalled conditions than in the signaled conditions. Additionally, the re-introduction of signals failed to produce a replication of the center-key response rate in most conditions. For example, for subject A1, when 5-s delays were in effect, the first signaled condition had a response rate of 78 key pecks per minute, and the second signaled condition had a response rate of 84 key pecks per minute. Likewise, there was a failure to replicate the center key response rates in the 5-s condition for subject A2 (84 and 118 responses per minute), the 1-s (43 and 64 key pecks per minute) and 5-s (54 and 79 key pecks per minute) signaled conditions for subject B3, and the 1-s and 5-s conditions for subject B4 (88 and 92 key pecks per minute). Thus, for most subjects in most conditions, the center-key response rate was not recovered in the second signaled condition.

Center-key reinforcement. Reinforcement rates are presented in Figure 11 and Table 4. In general, as the delay increased, reinforcement rate decreased. For subject A1, for example, 
reinforcement rates of 5.17 food presentations per minute were obtained in the 1-s delay conditions, 4.53 food presentations per minute were obtained in the 5-s delay condition, 4.41 food presentations per minute in the 10-s delay condition, 2.84 food presentations per minute in the 15-s delay condition, and 3.24 food presentations per minute in the 20 -s delay condition. Thus, for all but the 20-s delay condition, a decreasing trend was observed for subject A1 across the increasing delays when the rates were averaged across signaled conditions. For subject A2, a similar pattern was observed, with decreases in reinforcement rate across signaled conditions with increasing delay: rates of 5.50, 4.31, and 2.39 reinforcers per minute were obtained in the condition with 1-s, 5-s, and 10-s delays, respectively. For subject B3, when the signals were present, the reinforcement rates were $2.37,3.04$, and 3.15 reinforcers per minute in the $1-\mathrm{s}, 5$-s, and 10-s delay conditions, respectively. Thus, for subject B3, there was an increase in reinforcement rate with the increasing delays. Because the programmed delays following an escape response are excluded from the calculation of reinforcement rates, the decrease in reinforcement rates suggests an increase in persistence. That is, responding more (i.e., persisting, responding less efficiently) necessarily requires additional time. Because ratio schedules were in effect, increased response requirements are necessarily correlated with lower rates of reinforcement.

The removal of signals resulted in lower reinforcement rates. For example, for subject A2, the reinforcement rate was 4.18 reinforcers per minute in the unsignalled 1-s delay condition, and 5.50 reinforcers per minute in the presence of the signals. In the first 5-s delay signaled condition, 4.73 reinforcers per minute were obtained, and 4.49 reinforcers per minute were obtained in the unsignalled condition. Similar patterns were observed for the other subjects, as well, with a lower reinforcement rate in the unsignalled conditions relative to the signaled 
conditions (cf. Figure 11, and Table 4). The removal of the signals, then, was also accompanied by a lower obtained rate of reinforcement.

The patterning of reinforcer delivery is presented in Figure 12. In this figure, stacked bar graphs display the proportion of reinforcers delivered in each ratio. White bars indicate escapes that occurred when the lowest ratio was in effect, gray bars indicate escapes that occurred when the second ratio was in effect, and striped bars indicate that the third or fourth ratios were in effect. Labels indicate whether signals were present or absent and the delay to the onset of the next trial following an escape response. Data are from the final 9 stable sessions.

Most reinforcers were delivered following the completion of the lowest ratio (FR 10) in the signaled conditions (white bars). For example, for subject A1, 87\% of reinforcers were delivered after this ratio was completed across all sessions. In signaled conditions, $90 \%$ of reinforcers were delivered after completion of the first ratio, and $10 \%$ from the second ratio for subject A1. In the unsignalled conditions, $78 \%$ of reinforcers were delivered following the first ratio, $11 \%$ from the second, $6 \%$ from the third, and $5 \%$ from the fourth, largest ratio. In the absence of the signals and with increasing delays, more reinforcers were earned following completion of one of the higher ratio values (gray bars, striped bars) than when signals were present and delays were low. For example, for subject A1, successive unsignalled conditions (5 s, $10 \mathrm{~s}, 15 \mathrm{~s})$ were accompanied with increases in reinforcers following ratios other than the smallest ratios (e.g., 9\%, 34\%, 45\%, respectively; see the fifth, eighth, and eleventh bars of the top panel of Figure 12). This pattern of reinforcer delivery was consistent across all subjects (e.g., sixth through ninth bars of the second panel, second and seventh through ninth bars of the third panel, fifth bar of the bottom panel). The observed shift in reinforcer patterning is indicative of increased persistence in these conditions. 
Figure 12.
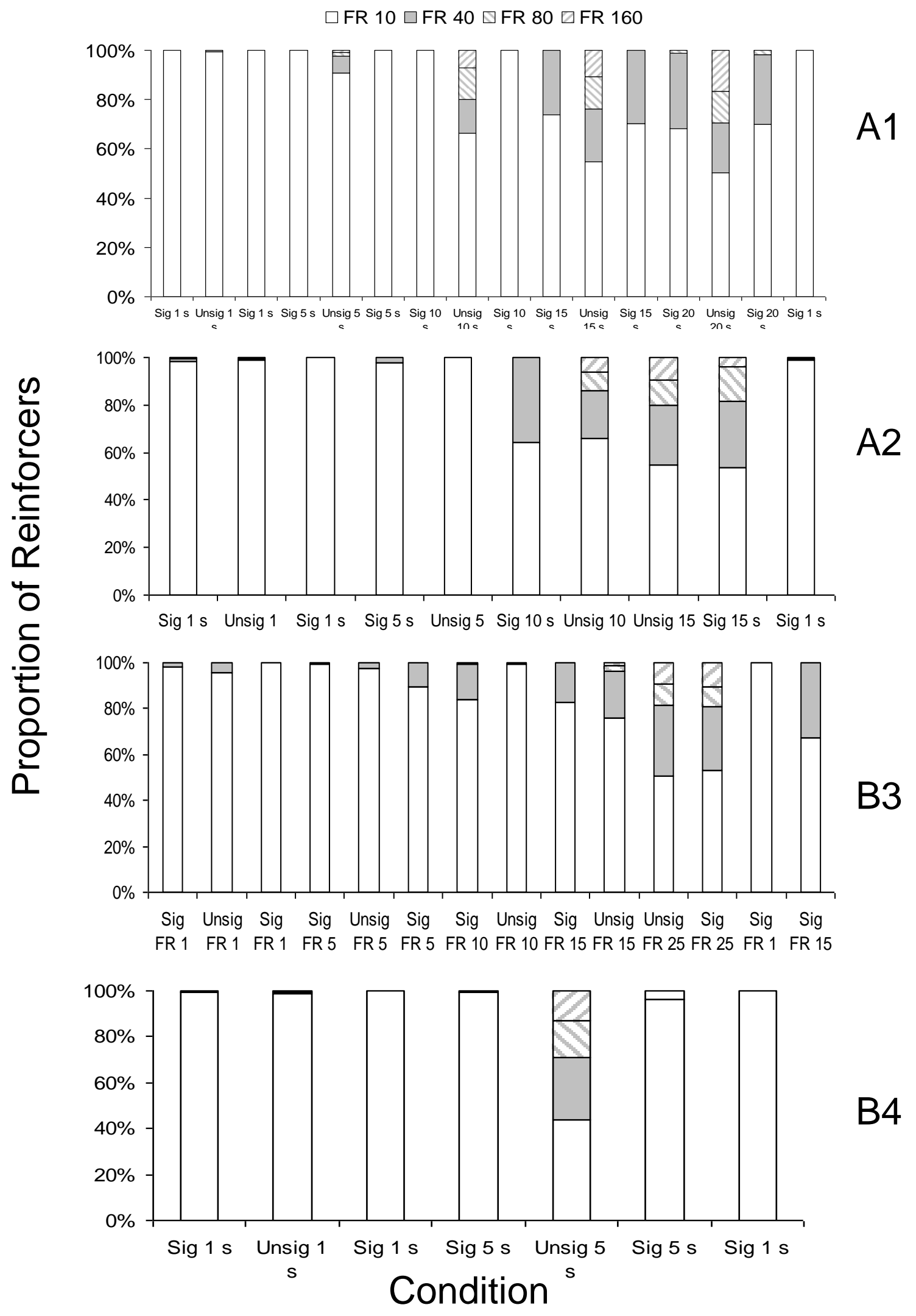
Efficiency index. The efficiency index for each subject in each condition is presented in Table 4 and Figure 10. Generally, when signals were present (white bars) and escape-ratio requirements were relatively low, the efficiency index was close to 1.0 (range: $1.00-1.05$ ), indicating that approximately 20 responses were emitted for each food delivery. This value (1.0) may be taken as an index of efficient responding, indicating that escapes occurred following the initial stimulus change. With the increasing delays, the efficiency index in the signaled conditions generally did not change until a particular value was reached (e.g., $15 \mathrm{~s}$ for subject A1, with a mean efficiency index of $1.64 ; 5 \mathrm{~s}$ for A2, with a mean efficiency index of $1.11 ; 10 \mathrm{~s}$ for B3, with a mean efficiency index of 1.53). This effect was not observed for subject B4, but this may be due to the fact that the effects of longer delays (i.e., greater than $5 \mathrm{~s}$ ) were not evaluated with this subject because the escape response was completely suppressed when the 5-s delay was used. The maximum observed efficiency index values in the signaled conditions were 1.69 for subjects A1 and B3, 2.10 for subject A2, and 1.12 for subject B4. These values occurred in conditions with relatively long delays (e.g., 20 s, 15 s, 10 s, 5 s, for subjects A1, A2, B3, and B4, respectively). When the 1-s delays were reinstated in the final condition, efficiency index values returned to their initial level for all subjects (range: $0.93-1.07$ ).

When the ratio changes were unsignalled, response efficiency was decreased. All 13 of the unsignalled conditions (Table 4 and Figure 10, gray bars) were accompanied by increases in the efficiency index relative to the signaled conditions with the same delay value. For example, for A1, the mean efficiency index was 1.05 for the signaled 1 -s conditions, and 1.36 for the unsignalled 1-s condition. Similar differences were observed across all other conditions for this subject., and across subjects, as well. For all conditions, higher efficiency index values were obtained in the unsignalled conditions relative to signaled conditions. Increases in the efficiency 
index reflect an increase in completion of ratios other than the initial, lowest ratio.

Escape responding. Increasing the delay following an escape response led to systematic changes in the frequency and patterning of escaping. The number of escape responses in each condition is presented in Figure 13 and Table 4. White bars indicate signaled conditions, and gray bars indicate unsignalled conditions. The labels indicate the delay following an escape response. Data are from the final 9 stable sessions, and error bars represent \pm SEM.

During signaled conditions, escaping generally decreased with an increasing delay to the onset of the next trial. When the 1-s delay was in effect, each subject escaped approximately 80 times per session (range: $81.78-82.11$ ). With an increase to a 5-s delay, the number of escape responses did not significantly change (mean $=82.00$ escapes per session, range: $75.56-84.89$ ). For the three subjects experiencing 10-s delays, the average number of escape responses per session decreased to 49.22 (range: 33.78 - 75.56). Only subject A1 experienced 15 -s and 20-s delays, and the mean number of escapes per session were 41.00 and 25.78 , respectively. Thus, in general, when looking at group means (which are representative of individual-subject data), the number of escape responses systematically decreased as a function of the delay duration in the signaled conditions.

The decreasing number of escapes as a function of increasing delay was especially salient in the unsignalled conditions (Figure 13, gray bars). For subjects A2 and B4 (second and bottom panels), as the delay increased, the number of escape responses systematically decreased in the unsignalled conditions. For example, subject A2 escaped an average of 95.89 times per session in the 1-s delay condition, 74.90 times per session in the 5-s delay condition, and 8.00 times in the 10-s delay condition. Subject B4 escaped 140.11 times per session in the 1-s delay condition (which is an increase from the signaled condition value of 81.78), and an average of 0.57 times 
Figure 13.
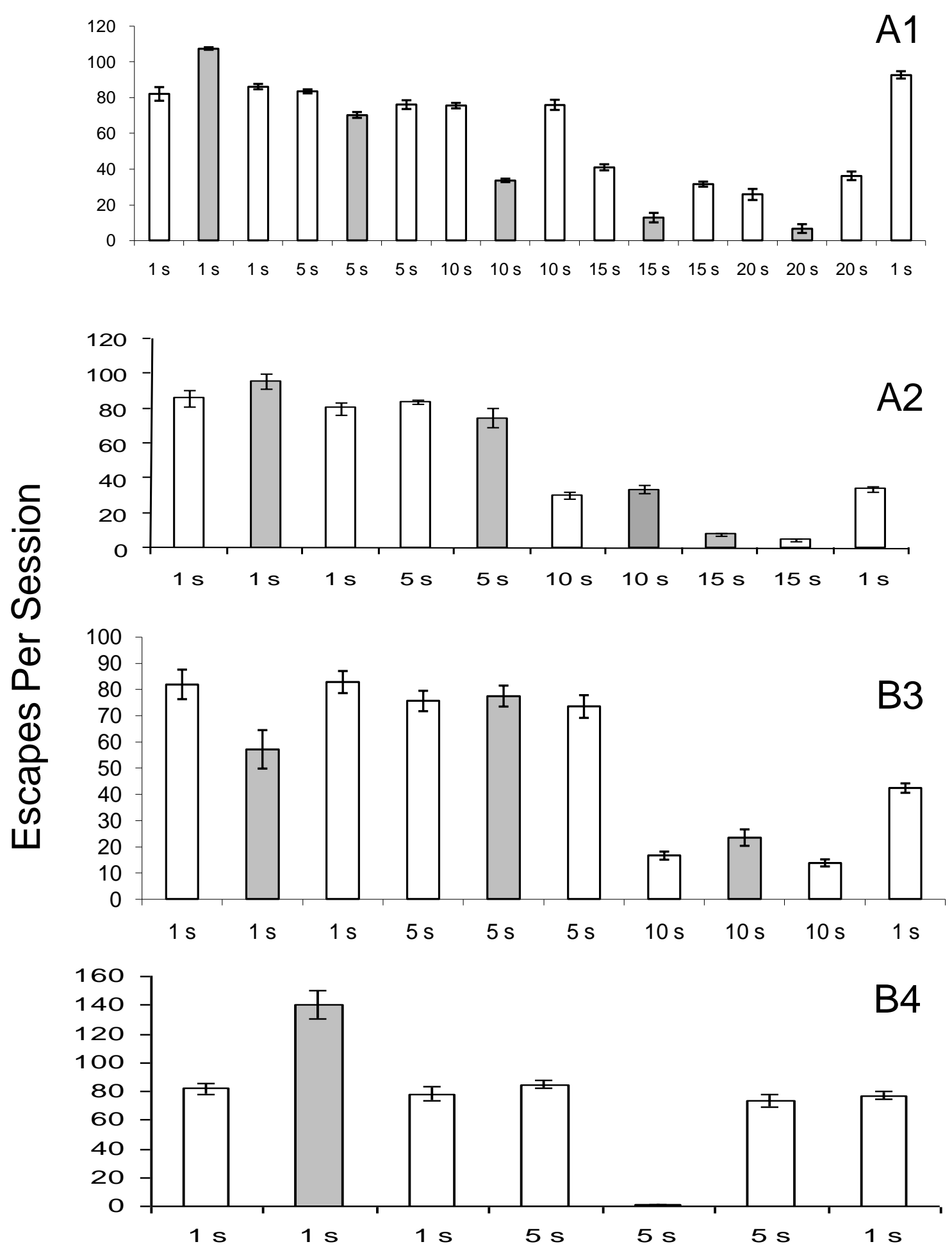

Condition 
in the 5-s delay condition. Subject B3 (third panel) exhibited an increase in escape responses between the 1-s delay condition (54.11 escapes per session) and the 5-s delay condition (74.33 escapes per session), but decreased in the 10-s delay condition (23.44 escapes per session). For subject A1 (top panel), the number of escape responses also increased in the unsignalled condition with 1 -s delays $($ mean $=107.56$ escapes $)$ relative to the signaled condition $($ mean $=$ 82.11 escapes), and escape responding decreased further in the unsignalled conditions with 5-s delays $($ mean $=70.33), 10$-s delays $($ mean $=33.67), 15-$ s delays $($ mean $=12.78)$, and 20-s delays $($ mean $=6.67)$. Thus, the number of escape responses systematically decreased as a function of the increasing delay. The greater decrease in escape responding in the unsignalled conditions compared to the signaled condition suggests an interaction between the delay and the signals.

As a second means of examining escape responding, Figure 14 contains stacked bar graphs presenting the distribution of escape responses across ratio values. White bars indicate escapes that occurred when the lowest ratio was in effect, gray bars indicate escapes that occurred when the second ratio was in effect, and striped bars indicate that the third or fourth ratios were in effect. Labels indicate the presence or absence of the signals (Sig/Unsig) and the delay following an escape response. Data are from the final 9 stable sessions.

Overall, most escapes occurred during the second ratio (white bars). When the signals were present, most escape responses occurred following the first stimulus change. For example, $78 \%, 89 \%, 95 \%$, and $99 \%$ of escapes occurred during the second ratio (i.e., after the first stimulus change) for subjects A1, A2, B3, and B4, respectively. Inspection of individual-session data reveal that nearly all of the escape responses during the second ratio typically occurred within 3 center-key responses after the stimulus change, i.e., immediately after the change in the center key color from white to yellow. 
Figure 14.

DFR 10 DFR 40 DFR 80 DFR 160

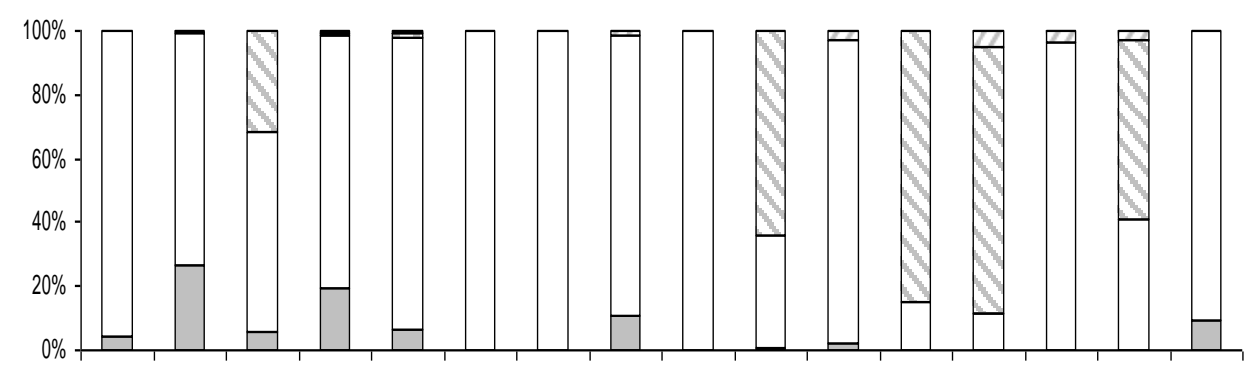

A1

Sig 1 s Unsig 1 Sig1s Sig 5 s Unsig 5 Sig 5 s Sig10s Unsig Sig10s Sig15s Unsig Sig15s Sig20s Unsig Sig 20s Sig $1 \mathrm{~s}$ $\begin{array}{lllll}s & S & 10 s & 15 s & \end{array}$ $20 \mathrm{~s}$

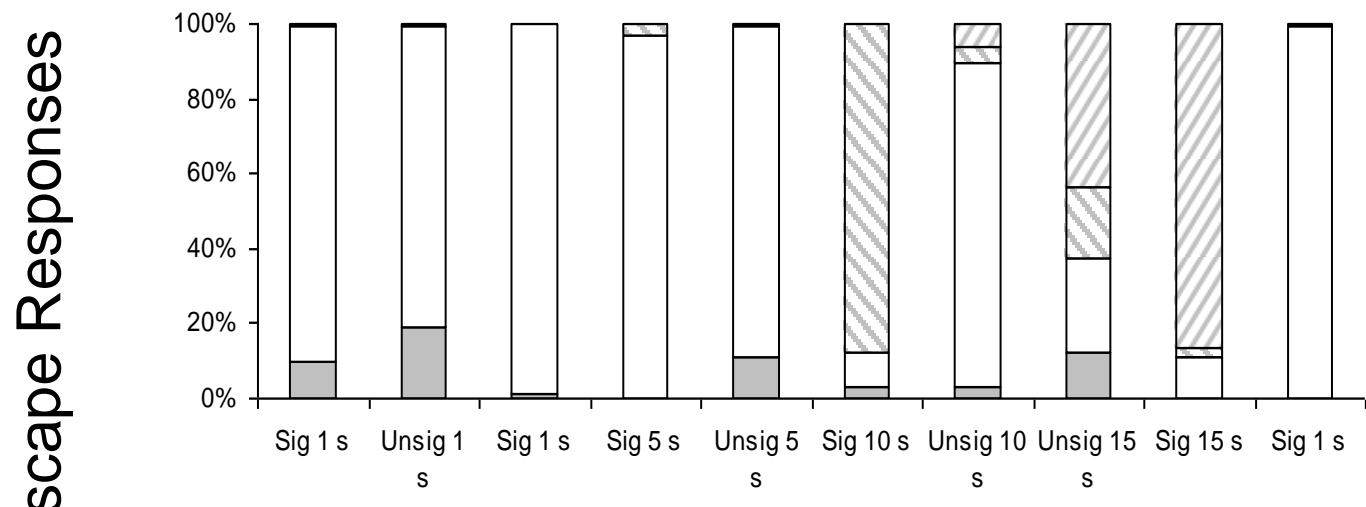

A2
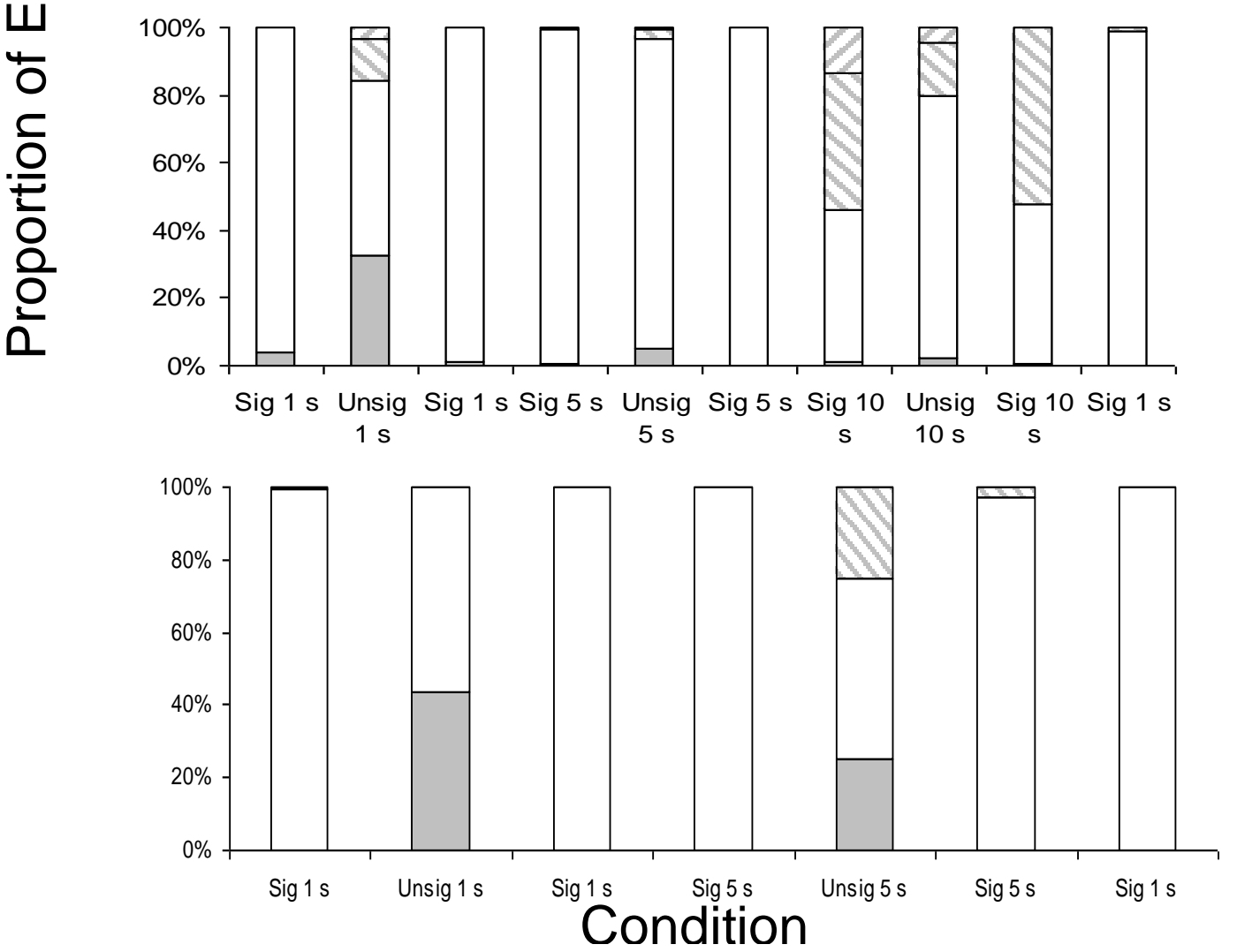

B4 
In the absence of the signals (i.e., when the key was always white), the number of escapes during the first ratio increased, as indicated by the white bars. For example, for subject A1, an average of $82 \%$ of escapes occurred during the second ratio, with $17 \%$ of escape responses occurring during the first ratio (gray bars) in the unsignalled conditions. Similar patterns were observed for the other subjects. As in Experiment 1, the increase in escapes during the first ratio was a function of a burst of escape responding. That is, series of escape responses (without intervening center-key responses) occurred. The changing escape proportion across delay and signals was generally consistent across subjects (see Figure 14). The increase in the early escapes in the unsignalled conditions suggests that lack of discrimination led to inefficient responding, with more escapes made before the first ratio was completed (i.e., subjects could not discriminate that the first ratio was still in effect). Inefficient responding in unsignalled conditions (compared to signaled conditions) was also supported by the efficiency index, as described above.

Condition reversal. As a final manipulation in this experiment, the delay value on the escape key was returned to $1 \mathrm{~s}$ to determine the extent to which a history of relatively long delays influences behavioral persistence when the delay is shortened. For subject A1, behavior recovered with respect to the number of escape responses (82.11 initially, 92.78 in the last condition) and the reinforcement rate (5.28 and 5.99 reinforcers per minute in the first and second 1-s condition, respectively), but there were larger differences between response rates ( 82 key pecks per minute initially, 97 key pecks per minute in the final condition). Subject A2 had more escape responses and higher rates of reinforcement in the second condition (86.11 and 5.84, respectively) than in the first (33.89, and 5.12), and lower efficiency index values in the second condition (0.93) than in the first (1.05). For subject B3, differences were observed with response rates ( 43 key pecks per minute in the first condition, and 91 key pecks per minute in the 
second condition), escapes (81.89 and 42.33 escapes per session in the first and last conditions, respectively), and reinforcement rate (2.47 and 6.33 reinforcers per minute in the first and last conditions, respectively). However, no difference was observed between the efficiency index values during the first (1.00) and last (1.07) 1-s delay conditions. For subject B4, higher rates of both responding and reinforcement in the second condition (98.70 and 6.09, responses and reinforcers per minute, respectively) than in the first condition (72.78 and 3.76 responses and reinforcers per minute, respectively) were obtained. Taken together, the various dependent measures suggest that a reversal was not achieved with the return to the lower delay values, unlike Experiment 1, in which a reversal occurred. The history with the longer delays seems to have influenced performance in subsequent conditions.

\section{Comparisons between experiments.}

A primary goal of this experiment was to directly draw comparisons between VR and FR schedules of reinforcement in the sunk-cost procedure used by Navarro and Fantino. To this end, the average number of escapes and center-key response rates in the signaled FR-1 and 1-s conditions were compared. The average number of escape responses per session was 83.56 (range: 54 - 111) in the FR-1 condition and 82.97 (range: 59 - 110) in the 1-s condition, i.e., escape responding was the same across groups. There was, however, a difference observed between the average center-key response rates, with 93 key pecks per minute in the FR-1 condition (range: 77 - 164) and 71 key pecks per minute in the 1-s condition (range: 26 - 93). Thus, the subjects with the VR schedules programmed on the center key had higher response rates than the subjects with the FR schedules. Although higher rates of responding were engendered, differences in the number of escapes were not observed. In terms of escape performance, then, the arrangements of the ratio values did not differentially affect behavior. 
A second goal of this experiment was to assess the effects of increasing delay as a control for increasing effort in Experiment 1. To explore the relative effects of each of these variables, the patterning of escapes and reinforcers in each experiment were examined. Increasing both delay and effort resulted in systematic decreases in escape responding (see Figures 5 and 13). Significant suppression of the escape response was observed with ratio values between 15 (subject B2) and 20 (subject B1) responses and delays between $5 \mathrm{~s}$ (B4) and $10 \mathrm{~s}$ (B3, A1, A2). For all subjects, complete suppression of the escape response (i.e., fewer than 4 escapes per session) was observed in Experiment 1. By contrast, only two subjects (A2 and B4) exhibited complete response suppression (i.e., fewer than four escapes per session) in the delay condition (with 15-s delays and 5-s delays, respectively).

\section{Discussion}

The sunk-cost effect occurs when individuals use previous expenditure to explain or justify contemporary decision making, rather than considering future costs or benefits. Sunk-cost behavior may be maladaptive in that it is a failure to optimize reinforcement, wasting resources while other (perhaps more profitable) courses of action are not pursued. The present experiment was designed to expand the model of the sunk-cost effect developed by Navarro and Fantino (2005). In the present experiment, effects of increasing the delay between an escape response and the onset of the next trial were examined. In this procedure, the most efficient or optimal course of action (i.e., associated with the lowest response requirement per reinforcer) was to escape. By contrast, persisting and continuing to respond in a progressively worsening situation was maladaptive (i.e., inefficient). With increasing delays, the likelihood of escape responses decreased, and, therefore, the sunk-cost effect (i.e., suboptimal persistence) became more likely. More reinforcers were obtained after satisfaction of higher center-key ratios (rather than lower 
center-key ratios) when delays were long than when delays were short. The changes observed in this experiment were consistent with the changes observed in Experiment 1, in which the response requirement on the escape key was systematically increased.

Based on the patterning of escape responses (see the final paragraph in the Results section), it may be concluded that the response requirement was a stronger determinant of persistence than the delay values. That is, more instances of complete escape-response suppression were observed with the escape requirement than with the delay. Thus, although increasing delay did suppress escape responding, under the parameters tested in the present experiment, it seems that this suppressive effect was relatively weak compared to the effect of the escape requirement. The relatively greater influence of effort relative to delay is consistent with other results (e.g., Grossbard \& Mazur, 1986; Tsunematsu, 2001). For example, in a delaydiscounting paradigm, Grossbard and Mazur (1986) demonstrated that there are differences between patterns of discounting based on effort and delay. To accomplish this, they yoked the delay to the delivery of the reinforcer to the time required to make the responses in an effortdiscounting condition. Within this procedure, Grossbard and Mazur determined that the degree of discounting due to effort was greater than that due to delay. More recently, Tsunematsu (2001) found that, in an experiment comparing effects of effort and delay (in the form of FR and FI schedules, respectively) in a closed economy, food intake (by pigeons) decreased more rapidly when the effort requirement was increased, relative to when the delay was increased. The results from the present study (i.e., the relatively greater impact of effort than delay) support the generality of the findings of this previous research.

With increasing delays, more reinforcers were delivered following the completion of higher (instead of lower) ratio requirements than when the delays were short. As the delay 
increased, although there were more reinforcers delivered following completion of higher ratio requirements than when delays were short, there was not a similar increase in escape responses. That is, once subjects began to persist, they continued to do so until food was delivered, even if completion of the lower ratios (e.g., FR 40, FR 80) did not provide access to grain. When escapes occurred in the conditions with longer delays, they did so most frequently in the second ratio (see Figure 13, white bars). With increasing delays, the proportion of escapes occurring later in the trial increased (Figure 13, striped bars). In the unsignalled conditions, the lack of discriminability may have led subjects to persist, as there were no external stimuli indicating the current ratio requirement or the number of responses remaining. This persistence was indicated by the increase of higher-ratio escapes, relative to when the signals were present. Likewise, efficiency index values increased in the unsignalled conditions relative to the signaled conditions, indicating an increase in persistence, and, therefore, less efficient responding. The lack of discriminative stimuli increased the uncertainty of the situation, making it less clear that the lower ratio requirements were not in effect. The increased uncertainty (i.e., lack of discriminability), in turn, led to less efficient responding, i.e., an increased likelihood of persistence. In this case, the persistence was suboptimal, in that more responses were made on the center key than would be required to receive food if an escape response was made.

The decreased response efficiency reflects the suppression of the escape response. It is possible that the delay itself may have been sufficiently aversive to eliminate escape responding, as was the increased effort to escape in Experiment 1. As the delay to the presentation of a reinforcer increases, that reinforcer loses its efficacy. This is a fundamental principle of reinforcement, and may be seen in the delay-discounting literature (cf. Ainslie, 1975; Diller, Saunders \& Anderson, 2008; Mazur, 1987) and in other areas as well (cf. Beardsley \& Balster, 
1993; Lattal, 1984; McDevitt, \& Williams, 2001; Stretch, Gerber \& Lane, 1976). Increasing the delay to the onset of the next trial should decrease responding that is followed by the delay.

One purpose of this study was to evaluate the relation between the increased response requirement and its accompanying delay. As noted above, similar escape patterns were observed with and increasing delay as when the response requirement was increased. If delay was an ineffective variable, the response patterns should not have changed as a function of its introduction. Thus, the changes observed in the first experiment may have been due, in part, to the delay that accompanied the response requirement. It is important to note that the number of responses required in Experiment 1 could be made before the programmed delays in Experiment 2 elapsed (i.e., the delays were longer in Experiment 2 than they would be in Experiment 1; see the response rate data in the Tables). Stated differently, the obtained delays in Experiment 1 were shorter than in Experiment 2. Since, for some subjects, complete suppression was observed when longer delays (e.g., $20 \mathrm{~s}$ ) were in effect, it is possible that the suppression of escape responding in Experiment 1 was due primarily to effort and not just the delay to the onset of the next trial. That is, because escaping continued in the presence of short delays in Experiment 2, the obtained delays in Experiment 1 may be unlikely to suppress responding. It was only when delays became sufficiently long (e.g., $20 \mathrm{~s}$ ) that escaping was suppressed for some subjects. For others (e.g., B4), relatively brief delays ( $5 \mathrm{~s}$ ) were sufficient to suppress escape responding, indicating a potential role for individual subject differences.

Under the parameters examined within these experiments, it seems that increasing effort was more effective than increasing delay in terms of suppressing escape responding and, therefore, increasing suboptimal persistence, consistent with previous findings (e.g., Tsunematsu, 2001). Further increasing the delay to the onset of the next trial would likely result in further 
decreases in escaping. Thus, in the present experiments, both effort and delay were effective variables, but the impact of effort may have been greater than that of delay.

The relative suppressive contribution of effort may have been greater than that of delay. Complete suppression was observed in more subjects with increasing ratio requirements than with increasing delays, delay still was an effective variable within this procedure. Indeed, it has been well established that increasing the delay to a reinforcer reduces its efficacy (cf. Ainslie, 1975; Diller, Saunders \& Anderson, 2008; Lattal, 1984; Mazur, 1987; McDevitt, \& Williams, 2001; Stretch, Gerber \& Lane, 1976). Future research could yoke the delay and the response requirement to more completely assess the relation between these variables.

That delay was an effective variable in the present study may have implications for sunkcost situations occurring outside of the laboratory. In recent research on the sunk-cost effect with younger and older adults, for example, Strough et al. (2008) presented a written vignette related to paying different amounts ( $\$ 0$ or $\$ 10.95$ ) to watch a bad movie. Participants were asked how much longer they would continue to watch the film. Older adults were less likely than younger adults to commit the sunk-cost fallacy, i.e., continue watching the movie based on past investment, implicating age as another potential determinant of the sunk-cost effect. In this example, should an individual stop watching the movie, there would necessarily be some delay before the onset of the next leisure activity—finding something else to watch or do would take some time. Even the classic economic example of initially investing in a poorly performing stock and deciding to continue investing or change the course of action contains a delay-before it is possible to invest in another stock, the alternative investment would have to be identified and the funds would have to be transferred. Thus, delay to the onset of the next opportunity to behave (invest, engage in leisure, peck a key) may have a great deal of relevance to sunk-cost behavior 
in and out of the laboratory. Strough and colleagues (2008) suggested that the impact of delay may change across the lifespan, as indicated by a greater likelihood of the sunk-cost effect by younger adults (i.e., college students) than by older adults. Delay, then, has consistently been shown to be an influential variable in the sunk-cost effect.

Another important finding from this experiment was that the ratio schedules used in this procedure (FR or VR) did not affect escape performance when delay, response requirements, and signals were held constant. There were, however, differences in response rate accompanying these different schedules. This difference is not unexpected, and it is well established that variable schedules of reinforcement result in higher response rates than fixed schedules (cf. Ferster \& Skinner, 1957).

\section{General Discussion}

The sunk-cost effect is the increased likelihood of persistence following an initial investment of some commodity, especially in progressively worsening situations. This type of persistence is viewed as maladaptive in that resources are wasted and more fruitful ventures are not pursued. If this pattern of persistence is extended in time, it may lead to clinically significant problems (Leahy, 2000). Although there have been a number of studies examining the sunk-cost effect in humans (e.g., Arkes \& Ayton, 1999; Arkes \& Hutzel, 2000; Bornstein \& Chapman, 1995; O’Flaherty \& Komaki, 1992; Strough et al., 2008), relatively few studies have examined this effect in non-human animals (cf. Navarro and Fantino, 2005; Pompilio, Kacelnik, \& Behmer, 2006). The studies with non-human animals have identified the degree and nature of the investment and the discriminability of the outcomes as important determinants of sunk-cost behavior. The present experiments add to this body of literature, implicating the effort to escape from the initial investment and the delay to the onset of the subsequent trial as factors 
contributing to the sunk-cost effect.

In the present experiments, increasing ratio requirements or increasing the delay to a reinforcer decreased escape responding. In some sense, these experiments expand on a large body of previous literature in which delay and effort have been manipulated (e.g., Ainslie, 1974; Dunn, 1982; Grossbard \& Mazur, 1986; Tsunematsu, 2001). That is, effects of delay and effort on response patterning have been evaluated before. The novelty of the present experiments, however, lies in the conceptualization as a sunk-cost effect and the increasing complexity of the situation (e.g., the progressive schedule arrangement, the escape key). The present experiments expand the model of sunk-cost behavior designed by Navarro and Fantino (2005) by evaluating effects of VR schedules (rather than only FR schedules), and by manipulating delay following escape responses and effort required to complete an escape response. The addition of these variables may add to the external validity of this model, and further clarifies their role in this type of suboptimal persistence.

The sunk-cost effect, as described by Navarro \& Fantino (2005), was generally observed in these two experiments with pigeons pecking for food. That is, when an initial investment (in the form of key pecks) was made, subjects were likely to continue responding under the following conditions: (1) when increases in response requirements were not signaled within a trial, (2) when escaping resulted in a relatively long delay to the onset of the next trial, and (3) when the number of responses required to escape from the current trial was relatively high. Interestingly, both the delay and the response requirement were sufficient to increase the likelihood of the sunk-cost effect (i.e., attenuate escaping), and they did so systematically. The degree of suppression of escaping, however, was not equal for these variables in the parameters that were studied. Based on the results of these two studies, it may be possible to conclude that 
both effort and delay are important variables in sunk-cost behavior. Effort may be a more salient determinant of sunk-cost behavior than delay, however. That is, per unit increase, effort may result in more rapid suppression than delay. The step size (of either duration or ratio requirement) may be an important determinant of the patterns of escaping and persistence that are observed. That is, with a 1-s increase in delay, the escape response may continue to a higher terminal value than if a 15-s step size was used. Future research may examine the influence of such parameters on this model of the sunk-cost effect.

Consistent with Navarro and Fantino's (2005) study, variables that control behavioral persistence were identified in the present experiments. The present findings further demonstrate the influence of the controlling variables identified by previous research in this area. For example, Navarro and Fantino $(2005,2007)$ and McCain (1986) identified discriminability as an important factor in persistence. Arkes and Hutzel (2000) implicated the prescribed probability of success as an important variable, which, when the probability is specified, relates to the discriminability of the outcome, and Bornstein and Chapman (1995) identified the salience of the choice situation as an important variable. In the present experiments, the salience of the choice situation and discriminability were manipulated in two ways: with signals and with the type of ratio scheduling. The signals had an interactive effect with the delays or the response requirement, but the scheduling of the ratios (fixed vs. variable) did not have a comparable effect. That is, the response requirement and delay had their greatest suppressive effects on escape responding in conjunction with the removal of the signals. This interaction resulted in an increase in the degree of persistence in the escape-optimal conditions, reflecting an increase in suboptimal patterns of responding. Here, suboptimal responding is defined as making more responses per reinforcer than was required or necessary for food delivery. 
In general, the obtained results were similar to those presented by Navarro and Fantino (2005) in that the sunk-cost effect was manipulated as a function of controlling variables (e.g., signals). In the persistence-optimal conditions, subjects escaped infrequently, and the escape response was completely suppressed in the unsignalled condition of this phase. When these subjects moved to the escape-optimal phase, their behavior was generally consistent with the subjects that did not experience persistence-optimal conditions, although they were less likely to escape in unsignalled conditions. Thus, the prior exposure to persistence-optimal conditions was not sufficient to maintain behavioral persistence. This is similar to the enduring influence of early experience on the behavior of starlings, presented by Pompilio and Kacelnik (2005). In the present experiments, an extended history with persistence-optimal conditions may have yielded different results.

The type of schedule used (fixed vs. variable) did not significantly affect the likelihood of persistence. Navarro and Fantino $(2005,2007)$ used FR schedules of reinforcement in their initial development of this model of the sunk-cost effect. These authors found that the magnitude of the within-trial increase was a variable that could control sunk-cost behavior. For example, in their third and fifth experiments, Navarro and Fantino (2005) found an increased likelihood of persistence when the center-key ratio requirements (and accompanying probability statements) were those used in the present experiment $(10,40,80,160)$ relative to when the average number of responses per reinforcer was smaller (e.g., with values of 5, 50, 100, 220). Despite the possibility of individual larger ratio requirements in the VR conditions than in the FR conditions, escape patterning was the same between these conditions, but center-key response rates were higher when VR schedules were in effect. This difference in response rates may be attributable to the nature of variable and fixed schedules, in that variable schedules typically engender higher 
response rates.

The manipulation of the type of schedule in effect relates to the probability of success implicated by Arkes \& Hutzel (2000), in that, when a fixed schedule is in place, the number of responses required to satisfy that schedule does not change. In contrast, when a variable schedule is in place, the number of responses required to satisfy that schedule does change across trials. When variability is introduced, the probability of success (here, how many responses are required to satisfy a given ratio) becomes less discriminable than with a fixed schedule. The probability of success may be related to the degree of certainty associated with the desired outcome. The comparisons made in the present experiments failed to find a difference as a function of this variable. That is, the general pattern of responding on both keys was the same when either FR or VR schedules of reinforcement were used. Thus, the generality of the model developed by Navarro and Fantino was expanded to include the use of VR schedules.

Although Navarro and Fantino described their study as an animal model of the sunk-cost effect, there are some differences between how this effect is conceptualized in their work and in the human literature. For example, Strough et al. (2008) commented that the consideration of past expenditure in decision-making tasks qualified as the sunk cost effect if persistence was greater than in similar situations without prior investments. In the model proposed by Navarro and Fantino and employed in the present studies, the only non-investment conditions that can be compared to the conditions in which investments have been made are the trials in which food is delivered after the lowest ratio is satisfied, but this is not necessarily the same as having a situation in which no investment has been made, but a choice is presented between continuing with a given course of action or not continuing. Future research could further extend the model of the sunk-cost effect by adding a no-investment condition for comparison. 
As the literature based on the sunk-cost effect is growing, it may be desirable to delineate the relations between this procedure and other areas of research. To this end, three areas will be briefly discussed, as they relate to the current results: soft commitment, diminishing returns, and foraging.

Rachlin's notion of soft commitment suggests that, in general, behavioral patterns that are already underway are unlikely to be abandoned for an alternate course of action. This is similar to the sunk-cost effect, in that it is a previous investment of behavior that increases the likelihood of future persistence. In Siegel and Rachlin's (1995) study, the addition of a signal immediately before a choice was made between a smaller, sooner reinforcer and a larger, later reinforcer enhanced the degree of self-control that was observed. With the addition of the signal, the obtained reinforcement was maximized. The same may be said about the signal in the sunk-cost procedure used in the current experiments. In the presence of the signal during the escapeoptimal conditions, subjects were more likely to behave optimally. That is, when the signal was removed, subjects escaped less and emitted more responses per reinforcer than when the signal was present. The number of responses made per reinforcer in the unsignalled conditions was greater than the number of responses required to obtain food (i.e., was inefficient). A key difference between the sunk-cost and soft-commitment procedures, however, is that in the softcommitment procedure, responding on the alternative correlated with the larger reinforcer was enhanced with the addition of the stimulus change, and in the sunk-cost procedure, escaping from the present (worsening) situation is the behavior became more likely. Despite the differences in the type of efficient responding that is enhanced by the signal, the presence of discriminative stimuli increased the efficiency of responses, in that reinforcement rates increased. 
Interestingly, in Experiment 1, when the signal was removed in the persistence-optimal conditions, these two subjects escaped less than when the signal was present. So, in this phase of the experiment, the removal of the signal enhanced efficient responding. Navarro and Fantino (2005) did not assess the removal of signals under persistence-optimal conditions, but, three of their four subjects persisted (i.e., continued to respond) close to $100 \%$ of the time (range: $94-$ $100 \%$ of trials) and the fourth subject persisted in approximately $83 \%$ of trials in the persistenceoptimal conditions. The behavior of the fourth subject is consistent with the performance of the two persistence-optimal subjects in the present experiment. Perhaps the removal of the signals in persistence-optimal conditions would have resulted in a decrease of escaping for the fourth subject in Navarro and Fantino's study. The different effects of signals across these two experimental phases extends the literature on the sunk-cost effect.

A second area worthy of comparison is the work on diminishing returns. In the general diminishing-returns procedure, fixed and progressive schedules are concurrently available. Allocating responses to the progressive schedule results in an increase in future progressiveschedule requirements, and responding on the fixed schedule results in the resetting of the progressive requirement. In the sunk-cost procedure, the increase occurs on a trial-by-trial basis in a probabilistic fashion, and responding on one alternative does not prevent responding on the other alternative. In a diminishing-returns procedure, the increase is typically not probabilistic, and the changes may occur throughout a session without the option of resetting the response requirement.

Although it bears similarities to the sunk-cost procedure, Navarro and Fantino (2007) suggested that comparisons between diminishing returns and sunk-cost experiments are not fruitful because of procedural differences (i.e., the unit of resetting differs). Despite this criticism 
by these authors, there may be useful comparisons to be made. For example, Hackenberg and Hineline (1992) found that switching between alternatives occurred at low PI values when FI values were relatively short. This may be similar to the findings of the current experiments in that the subjects were more likely to persist (i.e., respond on the progressive schedule) when the escape requirement or delays were relatively large; at shorter values, the subjects were more likely to escape.

Studies in which foraging was examined may also be relevant to the present discussion of the sunk-cost procedure. As outlined in the introduction, two assumptions about the relation between these literatures suggest that: (1) behavioral persistence should vary as a function of the escape requirements, with increasing escape requirements resulting in increased behavioral persistence and (2) the point of escaping should maximize overall reinforcement. In both of the present experiments, as the response requirement on the escape key or the delay to the next trial were increased, and the rate of behavioral persistence also increased (i.e., the number of escapes decreased). Thus, the first assumption was supported.

The efficiency index may provide a measure of the degree to which the point of escaping resulted in a maximization of overall reinforcement (the second assumption). As described above, the efficiency index is the number of responses per reinforcer divided by the number of responses per reinforcer under conditions of most efficient responding; see the Data Analysis section of Experiment 1 for the derivation of this value. When response efficiency is evaluated using the efficiency index, maximization was not observed in all conditions. For example, in the unsignalled conditions, and when the delay or response requirements were increased, the efficiency index increased to more than double that of when the signals were present and the other values were relatively low, indicating less efficient responding. Based on the current 
experiments, then, it can be stated that reinforcement rates were maximized in some conditions (i.e., signals, low delay and escape-ratio values) as a function of escaping. Maximizing reinforcement may be taken as an indication of efficient responding, and relates directly to the maladaptive features of the sunk-cost effect. In the investment example of the sunk-cost effect, where investment in a failing fund continues, the reinforcer (i.e., profit) is not maximized. It is the failure to maximize the magnitude of profit that makes persistence suboptimal and changing the course of the investment (i.e., escaping) a desirable choice.

The present experiments comprised a parametric examination of variables relevant to the model of the sunk-cost effect described by Navarro and Fantino. Future research could assess the influence the present manipulations (i.e., ratio values for food delivery or escape production, delay to the onset of the next trial) using different parameters. Parametric manipulation could occur at the level of the change in step sizes of the ratios used in the basic food-production schedule (as in Experiment 3 of Navarro and Fantino, 2005), or in the escape requirement or the delay following the escape response. If the escape requirement or delay increased with a small step size (e.g., 1 response or 1 second), it is possible that the escape responses would continue longer than if a larger step size (e.g., 10 responses or 10 seconds) was used.

Even though the results of Navarro and Fantino were generally supported, the findings were not completely replicated. For example, when the signals were removed in the escapeoptimal conditions (which contained 1-s delays when escape responses occurred), three of the four subjects used by Navarro and Fantino in their Experiment 1 persisted $100 \%$ of the time (i.e., did not escape). In the present study, however, escape responses continued in the 1-s unsignalled condition of both experiments. It was only with increasing response requirements or delays that the escape response was suppressed. Interestingly, the fourth subject used by Navarro and 
Fantino failed to persist (i.e., continued to escape) in the absence of the signals, which is more consistent with the performance of the subjects in the present experiments. It bears repeating, however, that it was in the unsignalled conditions that the delay or response requirement had their largest effects, consistent with Navarro and Fantino's findings.

The discrepant results of the present study and the work of Navarro and Fantino may be due to the history of the subjects. Immediately prior to participating in the present experiments, subjects had experience responding on the center and side keys (in a choice procedure), but their experimental histories before that project were largely unknown. In contrast, Navarro and Fantino (2005) used a mixture of naïve and experienced pigeons across experiments (with all naïve birds in Experiments 1 and 5 and all experienced birds in Experiments 2 and 3), but did not specify the type of experience that they had. It is unlikely that the subjects used in the present experiment and the subjects used in the study by Navarro and Fantino had similar histories. Although it may be difficult to isolate, experimental history (or lack thereof) could be an important factor when considering the current results.

In Experiment 1 of the present set of studies, effects of increasing effort to escape from a sunk-cost situation were evaluated. In general, increasing the number of responses required to reset the ratio requirement decreased the likelihood that an escape responses would occur. Because more responses were required, the delay to the onset of the next trial was necessarily increased. Thus, Experiment 2 was designed to assess the contributions of delay to the observed decrease in escaping. One potential concern about the procedures used in this experiment may be that the delay correlated with the increase response requirement and the delay superimposed following the escape response were not equivalent. That is, based on mean response rates (presented in Tables 2-4), subjects frequently completed the required number of escape 
responses before the delay would have elapsed. The inequity of the delay and response requirement does not allow for the assessment of the respective effects of each. With sufficiently high response requirements, for example, it is possible that suppressive effects identical to those of obtained with the delay would be observed. Yoking the response requirement and the delay such that the delay value was based on how long (on average) it takes the subject to make the required number of responses would rectify this situation. The yoked arrangement has the advantage of examining effects of delay and the response requirement within a single subject, rather than between groups. The single-subject comparison would clarify effects of response requirement and delay, in that effects of these variables on escape responding could be compared within a single organism, removing the issue of different experimental histories.

Yoking provides one way to examine the relative importance of each variable (delay and effort). Even in a yoked situation, however, there is a difference in what is required of the subjects when the effort or delay is manipulated. That is, subjects have to work more when the response requirement is increased, but can engage in other behaviors during a delay. Given this difference, and the previously established greater suppressive effects of effort relative to delay in behavioral economics paradigms, such as the development of discounting functions (Grossbard \& Mazur, 1986) and a demand-curve analysis (cf. Tsunematsu, 2001), it is likely that the efficacy of effort would be greater than delay, even if the values were yoked.

Signaling changing ratio requirements on the escape key may also yield interesting results in the model of the sunk-cost effect. In the procedure used by Navarro and Fantino (2005) and in the present experiments, the escape key contained the same stimulus for the duration of the experiment, and this stimulus did not change as a function of the ratio that was in effect. In the present experiments, because limited stimuli were available, the escape key was illuminated 
white, which was the same color as the lowest ratio requirement (10). Adding signals to the escape key (e.g., changing color or flashing) would likely increase the discriminability of the current conditions and make the choice to continue or escape more salient (e.g., Bornstein \& Chapman, 1995). Increasing the salience of the choice situation may increase the likelihood that escape responses would occur and, therefore, increase the efficiency of responding. This further manipulation of the discriminability of the current situation would help to describe the limits of the influence of discriminative stimuli in this model of the sunk-cost effect.

Future research could also assess the generality of the effects of response requirement and delay with humans. In their 2007 study, Navarro and Fantino replicated their initial (2005) findings and underscored the importance of the discriminative stimuli in sunk-cost behavior. A similar replication of the present experiments would allow conclusions to be drawn about the generality of effects of delay and escape effort across species. Additionally, replications with other nonhuman species would also support the generality of the findings from the present experiment (e.g., Pompilio, Kacelnik, \& Behmer, 2006). The exploration of these variables across a variety of species and in a variety of settings and formats (e.g., human-operant preparation, written vignettes) would support the reliability and validity of the findings of the present study. Specifically, reliability would be supported if the same patterns of results were obtained across participants or settings, and validity would be supported if additional measures of maladaptive persistence (i.e., the sunk-cost effect) were correlated with the present experimental preparation. Strengthening the model through demonstrations of reliability and validity would provide additional evidence of the importance of the key variables.

The extension of the basic laboratory finding to the realm of human behavior is an important goal of behavior analysis (cf. Baer, Wolf, \& Risley, 1968; Skinner, 1971). While 
cross-species replication may enhance the validity of the findings of the present experiments, it is clear that manipulating the variables of response requirement, signals, and delay, resulted in changes in patterns of persistence. When one takes the position that the order observed in the current study is based on fundamental laws of behavior, it seems reasonable to suggest that these variables would exert similar control on human behavior. Based on the current results, then, it may be possible to develop a line of research that focuses on ways to enhance signals associated with worsening conditions, reduce the amount of effort required to escape from the worsening situation, and mediate the delay preceding the next opportunity to respond. If the critical variables are controlled in application, perhaps the likelihood of this type of maladaptive persistence could be minimized.

One procedure that has been developed to attenuate the effects of delay is tolerance training (cf. Schweitzer and Sulzer-Azaroff, 1988). In tolerance training, preschool children were taught to choose a larger, delayed reinforcer over a smaller, sooner reinforcer by presenting both the large and small reinforcers concurrently and gradually increasing the delay to the larger reinforcer. The goal of this procedure was to teach the participants to maximize the overall rate of reinforcement by consistently selecting the larger reinforcer. Perhaps an intervention that provides contact with a maximized rate of reinforcement through efficient responding and gradually increases the sunk cost could effectively teach individuals to avoid maladaptive behavioral persistence. Likewise, a similar intervention could be developed to gradually fade in effort, and to decrease the likelihood of maladaptive persistence.

\section{Conclusion}

The present experiments expanded on the work of previous studies (e.g., Navarro and Fantino 2005, 2007), further developing the behavior-analytic model of the sunk-cost effect with 
non-human animals. The results of the present experiments replicated the findings of Navarro and Fantino, and established that whether reinforcers are scheduled according to FR or VR schedules does not differentially affect escaping in this procedure. Additionally, the present experiments were the first to identify effort to escape and the delay following an escape response as important variables in the sunk-cost effect. Effects of effort and delay were modified by the presence and absence of the signals, underscoring the importance of discriminability in this paradigm. 


\section{References}

Ainslie, G. W. (1974). Impulse control in pigeons. Journal of the Experimental Analysis of Behavior, 21, 485-489.

Arkes, H., \& Ayton, P. (1999). The sunk cost and Concorde effects: Are humans less rational than lower animals? Psychological Bulletin, 125, 591-600.

Arkes, H., \& Hutzel, L. (2000). The role of probability of success estimates in the sunk cost effect. Journal of Behavioral Decision Making, 13, 295-306.

Baer, D. M., Wolf, M. M., \& Risley, T. R. (1968). Some current dimensions of applied behavior analysis. Journal of Applied Behavior Analysis, 1, 91-97.

Beardsley, P. M., \& Balster, R. L. (1993). The effects of delay of reinforcement and dose on the self-administration of cocaine and procaine in rhesus monkeys. Drug and Alcohol Dependence, 34, 37-43.

Bornstein, B., \& Chapman, G., (1995) Learning lessons from sunk costs. Journal of Experimental Psychology: Applied, 1, 251-269.

de la Piedad, X., Field, D. \& Rachlin, H. (2006). The influence of prior choices on current choice. Journal of the Experimental Analysis of Behavior, 85, 3-21.

Diller, J. W., Saunders, B. T., \& Anderson, K. G. (2008). Effects of acute and repeated administration of caffeine on temporal discounting in rats. Pharmacology, Biochemistry \& Behavior, 89, 546-559.

Dunn, R. M. (1982). Choice, relative reinforcer duration and the changeover ratio. Journal of the Experimental Analysis of Behavior, 38, 313-319.

Fantino, E. (2004). Behavior-analytic approaches to decision making. Behavioural Processes, $66,279-288$. 
Fantino, E. \& Abarca, N. (1985). Choice, optimal foraging, and the delay-reduction hypothesis. The Behavioral and Brain Sciences, 8, 315-330.

Ferster, C. B. \& Skinner, B. F. (1957). Schedules of Reinforcement. New York: AppletonCentury-Crofts.

Goltz, S. (1999). Can't stop on a dime: the roles of matching and momentum in persistence of commitment. Journal of Organization Behavior Management, 19, 37-63.

Grossbard, C. L. \& Mazur, J. E. (1986). A comparison of delays and ratio requirements in selfcontrol choice. Journal of the Experimental Analysis of Behavior, 45, 305-315.

Hackenberg T. D. \& Axtell, S. A. (1993). Human's choices in situations of time-based diminishing returns. Journal of the Experimental Analysis of Behavior, 59, 445-470.

Hackenberg, T. D. \& Hineline, P. N. (1992). Choice in situations of time-based diminishing returns: Immediate versus delayed consequences of action. Journal of the Experimental Analysis of Behavior, 57, 67-80.

Hineline, P. N. \& Sodetz, F. J. (1987). Appetitive and aversive schedule preferences: Schedule transitions as intervening events. In M. L. Commons, J. E. Mazur, J. A. Nevin \& H. Rachlin (Eds.), Quantitative analysis of behavior: Volume 5. The effect of delay and of intervening events on reinforcement value (pp. 141-157). Hillsdale, NJ: Erlbaum.

Ito, M. \& Fantino, E. (1983). Choice, foraging, and reinforcer duration. Journal of the Experimental Analysis of Behavior, 46, 93-103.

Jacobs, E. A. \& Hackenberg, T. D. (1996). Humans' choices in situations of time-based diminishing returns: Effects of fixed-interval duration and progressive-interval step size. Journal of the Experimental Analysis of Behavior, 65, 5-19.

Lattal, K. A. (1984). Signal functions in delayed reinforcement. Journal of the Experimental 
Analysis of Behavior, 42, 239-253.

Leahy, R. (2000). Sunk costs and resistance to change. Journal of Cognitive Psychotherapy, 14, $355-371$.

Lieving, G. A., Reilly, M. P., \& Lattal, K. A. (2006). Disruption of responding maintained by conditioned reinforcement: Alterations in response-conditioned-reinforcer relations. Journal of the Experimental Analysis of Behavior, 86, 197-209.

Mazur, J. E. (1987). In Commons, M., Mazur, J., Nevin, J., \& Rachlin, H. (Eds.) Quantitative Analyses of Behavior: The effects of delay and of intervening events on reinforcement value (pp. 55-73). Hillside, NJ: Lawrence Erlbaum Associates, Publishers.

McCain, B. E. (1986). Continuing investment under conditions of failure: A laboratory study of the limits to escalation. Journal of Applied Psychology, 71, 280-284.

McDevitt, M. A. \& Williams, B. A. (2001). Effects of signaled versus unsignaled delay of reinforcement on choice. Journal of the Experimental Analysis of Behavior, 75 165-182.

Moon, H. (2001). Looking forward and looking back: Integrating completion and sunk-cost effects within an escalation-of-commitment progress decision. Journal of Applied Psychology, 86, 104-113.

Navarro, A., \& Fantino, E. (2005). The sunk cost effect in pigeons and humans. Journal of the Experimental Analysis of Behavior, 83, 1-13.

Navarro, A. \& Fantino, E. (2007). The role of discriminative stimuli in the sunk cost effect. Mexican Journal of Behavior Analysis, 33, 19-29.

O’Flaherty, B., \& Komaki, J. (1992). Going beyond with Bayesian updating. Journal of Applied Behavior Analysis, 25, 585-597.

Pompilio, L. \& Kacelnik, A. (2005). State-dependent learning and suboptimal choice: When 
starlings prefer long over short delays to food. Animal Behaviour, 70, 571-578.

Pompilio, L. Kacelnik, A. \& Behmer, S. T. (2006). State-dependent learned valuation drives choice in an invertebrate. Science, 311, 1613-1615.

Rachlin, H. (2004). The Science of Self-Control. Cambridge, MA: Harvard University Press.

Schweitzer, J. B. \& Sulzer-Azaroff, B. (1988). Self-control: Teaching tolerance for delay in impulsive children. Journal of the Experimental Analysis of Behavior, 50, 173-186.

Siegel, E., \& Rachlin, H. (1995). Soft commitment: Self-control achieved by response persistence. Journal of the Experimental Analysis of Behavior, 64, 117-128.

Skinner, B. F. (1971). Beyond Freedom and Dignity. New York, NY: Bantam/Vintage Press.

Stretch, R., Gerber, G. J., \& Lane, E. (1976). Cocaine self-injection behaviour under schedules of delayed reinforcement in monkeys. Canadian Journal of Physiology and Pharmacology, $54,632-638$.

Strough, J., Mehta, C. M., McFall, J. P., \& Schuller, K. L. (2008). Are older adults less subject to the sunk-cost fallacy than younger adults? Psychological Science, 19(7), 650-652.

Tsunematsu, S. (2001). Effort- and time-cost effects on demand curves for food by pigeons under short session closed economies. Behavioral Processes, 53, 47-56.

Wanchisen, B. A., Tatham, T. A. \& Hineline, P. N. (1988). Pigeons' choices in situations of diminishing returns: Fixed- versus progressive-ratio schedules. Journal of the Experimental Analysis of Behavior, 50, 375-394. 